\title{
Further Developing Processing Techniques of Optical Satellite Images in the Context of Forest Monitoring
}

\begin{abstract}
Dissertation
to obtain the Ph.D. degree from the Faculty of Forest Science and Forest

Ecology,

Georg-August-University Göttingen
\end{abstract}

By

Dengkui Mo

Born in Jingzhou, Hubei Province, People's Republic of China 
Supervisor:

Prof. Dr. Christoph Kleinn

1. Referee/examiner: Prof. Dr. Christoph Kleinn

2. Referee/examiner: Prof. Dr. Yuanchang Lu

3. Examiner: $\quad$ Prof. Dr. Martin Kappas

Date of oral examination: July 2018 


\section{Table of Contents}

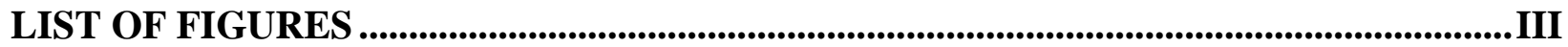

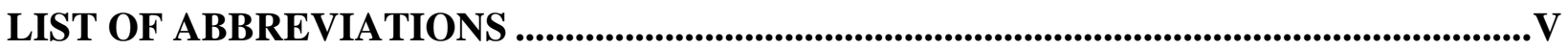

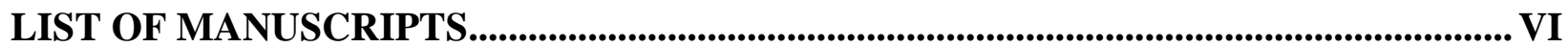

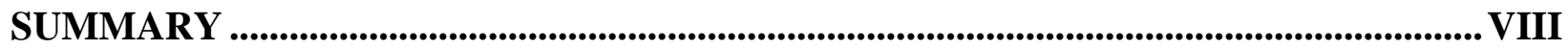

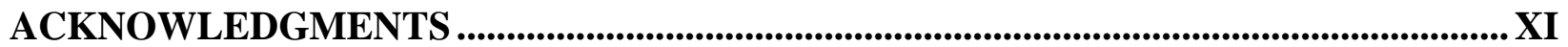

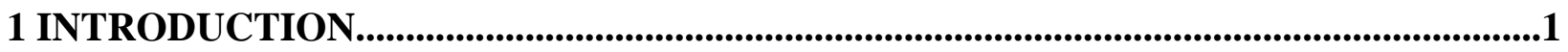

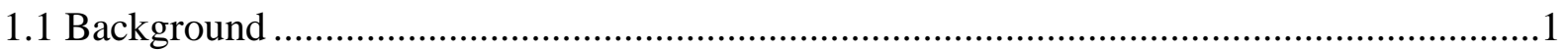

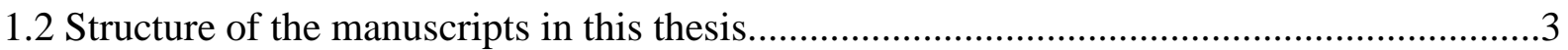

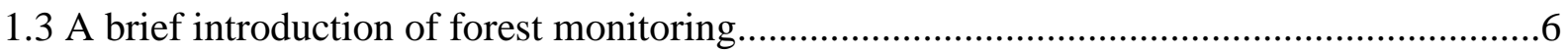

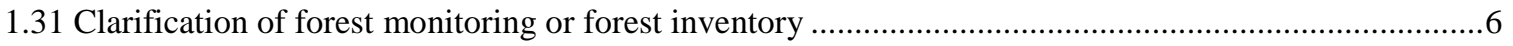

1.32 Exploring forest monitoring applications using remote sensing data ...........................................6

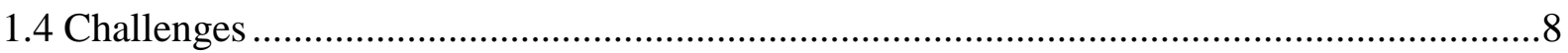

2 BACKGROUND OF FOREST CARBON MONITORING IN CHINA ..............................13

2.1 Forest carbon and sustainable forest management.............................................................13

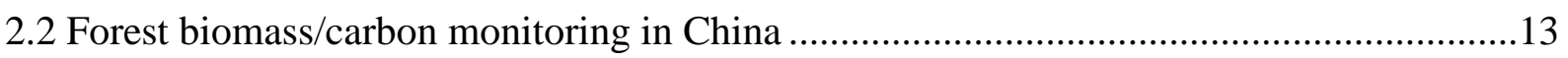

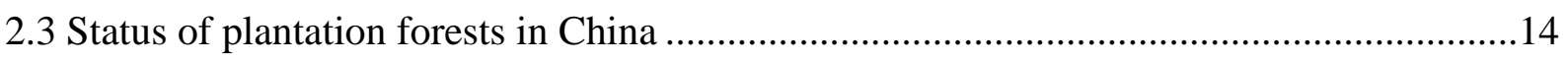

3 OVERVIEW OF THE LIN4CARBON PROJECT ..................................................................17

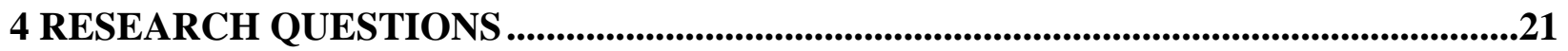

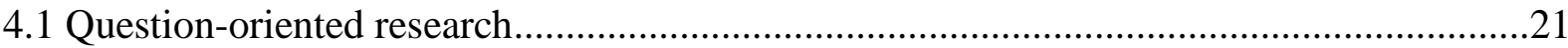

4.2 How to eliminate the spectral distortion caused by the variability of illumination

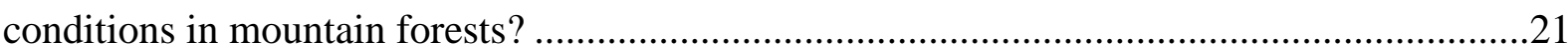

4.3 What is the NDVI response mechanism during illumination conditions during the year?.23

4.4 How to remove haze from optical satellite images to enhance data availability? ..............24

4.5 How to reconstruct high-spatial-resolution multispectral images using the panchromatic image as a reference making the size of field plot and pixel closer?....................................25

5 SYNTHESIS: FURTHER DEVELOPING PROCESSING TECHNIQUES OF OPTICAL SATELLITE IMAGES IN THE CONTEXT OF FOREST MONITORING ...........................27

5.1 Refined image processing techniques for severe atmospheric conditions ..........................28

5.2 Refined image processing techniques for rugged mountainous terrain ..............................30

5.3 Refined image processing techniques for scaling issues......................................................3

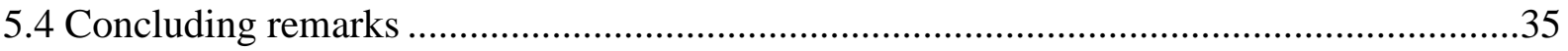




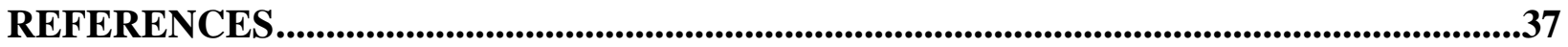

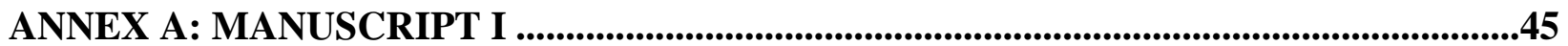

ANNEX B: MANUSCRIPT II ..............................................................................................................51

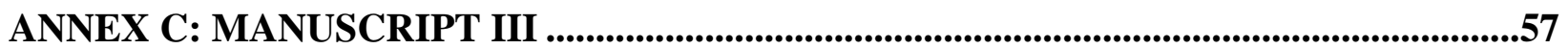

ANNEX D: MANUSCRIPT IV

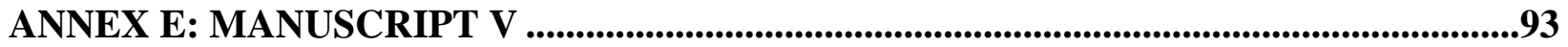

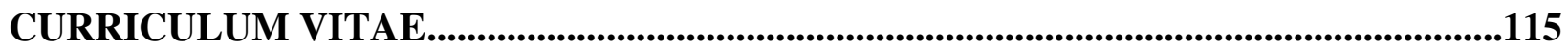




\section{List of Figures}

Figure 1. The framework of forest carbon monitoring techniques based on optical satellite imagery and field observations, and the five selected manuscripts that attempt to solve the challenges encountered during the implementation of the Lin4Carbon project..........4

Figure 2. Location of study site and illustration of terrain conditions produced from SRTM 1 Arc-Second Global simulated as shaded relief with sun elevation $=60^{\circ}$, and sun azimuth $=45^{\circ}$.

Figure 3. (1) Chinese fir (Cunninghamia lanceolate) plantation and (2) mixed natural forest from the study site. .20

Figure 4. Due to the variation of topography and illumination conditions, even the same type of land use has a variety of spectral characteristics. The pixels pointed by the red arrows are pure bamboo forests under different terrain and illumination conditions (image from Google Earth). .22

Figure 5. The image is highly visually consistent with the corresponding illumination conditions regarding light-dark variation. (1) Original Landsat 8 (Band composition $=$ Band 7, 5, 3, using 2\% linear stretch), (2) Illumination condition (IL). Note: Date acquired $=2014$ 03-30, Sun azimuth $=135.84^{\circ}$, Sun elevation $=55.65^{\circ}$ and DEM using SRTM 1 ArcSecond Global elevation data.

Figure 6. Example of before and after haze removal of optical satellite images (selected area of study site). (1) Original Landsat 8 (band composition (NIR, red, green), using $2 \%$ linear stretch, (2) haze removed image by our implementation using an algorithm from (Makarau et al., 2014b; Makarau et al., 2016). .25

Figure 7. Inventory plot size does not match the pixel size of the multispectral image. (1) Inventory plot design from Lin4Carbon Forest Inventory Guidelines Field Protocol, Dr. Lutz Fehrmann, August 2013, (2) pixel size of multispectral images of Landsat 8 OLI, $30 * 30 \mathrm{~m}^{2}$ .26 
Figure 8. The availability of optical satellite images is restricted by strong atmospheric variability, such as clouds, haze, etc. Compared to the difficulty of restoring cloud pixels, haze-covered pixels have the potential to be restored. In practice, haze-covered pixels are sometimes incorrectly detected as cloud pixels (see the area marked by the red polyline). (1) Original Landsat 8 false-color combination, (2) cloud detection for Landsat 8 is based on Fmask (Zhu et al., 2015). LANDSAT_SCENE_ID = "LC81230322016189LGN00"

Figure 9. Effect of illumination conditions caused by topography and solar light on reflectance. The reflectance or radiation in the shaded areas of the mountain forest is relatively low, while the effect in the irradiated area is the opposite. 


\section{List of Abbreviations}

\begin{tabular}{|c|c|}
\hline AGB & forest aboveground biomass \\
\hline DBH & diameter at breast height \\
\hline DEM & digital elevation model \\
\hline EVI & enhanced vegetation index \\
\hline GHG & greenhouse gas \\
\hline GSD & ground sampling distance \\
\hline IC & illumination condition \\
\hline IL & illumination \\
\hline IPCC & Intergovernmental Panel on Climate Change \\
\hline $\mathbf{k}-\mathbf{N N}$ & k-nearest neighbors \\
\hline LiDAR & light detection and ranging \\
\hline NASA & national aeronautics and space administration \\
\hline NDVI & normalized difference vegetation index \\
\hline NFI & national forest inventory \\
\hline REDD & reducing emissions from deforestation and forest degradation \\
\hline RSR & reduced simple ratio \\
\hline SAVI & soil-adjusted vegetation index \\
\hline SFM & sustainable forest management \\
\hline SLCP & sloping lands conversion program \\
\hline SRTM & shuttle radar topography mission \\
\hline TLS & terrestrial laser scanning \\
\hline UNFCCC & United Nations Framework Convention on Climate Change \\
\hline
\end{tabular}




\section{List of Manuscripts}

This dissertation is presented in a cumulative form and consists of five individual manuscripts. Each manuscript is sequentially marked with its corresponding Roman numeral. All manuscripts are reproduced in Annex A - E and were typeset according to the submission requirements of the target journals. My contribution makes up $80 \%$ of all five manuscripts and includes conceptual design, data collection, algorithmic development, data analysis and interpretation, drafting and revising of these manuscripts. Manuscripts I and II have been published, Manuscripts III and IV have been submitted, and Manuscript V is ready for submission.

\section{Manuscripts and their status:}

I. Moving Window-based Topographic Normalization of Optical Satellite Imagery for Forest Mapping in Mountainous Terrain

Dengkui Mo, Hans Fuchs, Lutz Fehrmann, Haijun Yang, Yuanchang Lu Christoph Kleinn

Status: published in the peer-reviewed conference of IEEE Earth Observation and Remote Sensing Applications (EORSA), 2014

II. Local Parameter Estimation of Topographic Normalization for Forest Types Classification

Dengkui Mo, Hans Fuchs, Lutz Fehrmann, Haijun Yang, Yuanchang Lu, Christoph Kleinn

Status: published in the IEEE Geoscience and Remote Sensing Letters

III. Effects of Intra-annual Illumination Conditions on NDVI in Mountainous Forest

Dengkui Mo, Lutz Fehrmann, Hans Fuchs, Paul Magdon, Christoph Kleinn

Status: Submitted to the IEEE Geoscience and Remote Sensing Letters 
IV. A Simple and Robust Haze Removal Method for VHR Optical Satellite Image Based on Mean Vector $\mathbf{L}^{2}$-norm

Dengkui Mo, Hans Fuchs, Lutz Fehrmann, Nils Nölke, Paul Magdon, Christoph Kleinn

Status: Submitted to Remote Sensing

V. A Novel Method of Panchromatic Image Weighted Multispectral Imagery Reconstruction

Dengkui Mo, Hans Fuchs, Lutz Fehrmann, Paul Magdon, Christoph Kleinn

Status: ready for submission 


\section{Summary}

The efficient monitoring of forests is essential in the fields of forest resource assessment, management, and scientific decision-making. Integrated remote sensing imagery and field observations are widely used and are considered highly efficient in the field of forest monitoring (such as the monitoring of forest stocks, biomass, forest carbon, and forest type maps) on a local, regional and even global scale.

In the field of forest monitoring by means of remote sensing, optical satellite imagery plays a critical role. However, for optical satellite image processing techniques applied to highprecision forest monitoring to be truly effective, a number of challenges still exist, such as the variability of the atmosphere, topography, illumination conditions and scale issues. To mitigate or eliminate the impact of these challenges, under the technical framework of the Lin4Carbon project, several refined image processing techniques have been proposed and have also been proven to be quite advanced techniques. This thesis consists of five manuscripts. Each manuscript is inspired by problems encountered in the implementation of the Lin4Carbon project. It is worth noting that in order to demonstrate the universality and advancement of these techniques, the locations and data are not entirely limited to the Lin4carbon project.

Manuscript I proposes a simple and straightforward, moving window-based, rotationcorrection topographic normalization model as the means to achieve improved forest mapping. An underlying assumption is that the same forest type has stable spectral characteristics, which are essential if forest monitoring is to be accurate. Our proposed model yields less intra-class heterogeneity and without the "overcorrection" phenomenon in vision when compared to uncorrected data or global correction methods.

Manuscript II further discusses the shortcomings of various topographic normalization methods based on global parameter estimations. In general, the band-specific methods lead to overcorrection, while land-cover-specific methods have difficulties in obtaining prior data on land types, and a variety of newly-advanced topographic normalization methods have difficulty achieving satisfactory results, especially when these methods are applied to sites with large areas and significant differences in landscape. Thus, this manuscript aims to establish a simple, generalized, standard, topographic normalization model for large-area optical satellite images. 
We assume that the relationship between the spectrum signature and the illumination conditions is specific to the site. Also, the empirical parameters of each pixel can be computed directly from a given window size. Compared with Manuscript I, this manuscript was given the same conclusions in the case of the use of new Landsat 8 images and new illumination conditions. In addition, we further tested our proposed model's performance on the classification of forest types. We conclude that the proposed method achieves higher classification accuracy and requires fewer training samples.

Manuscript III aims to help understand the mechanism of the dynamic effects of illumination conditions on the normalized difference vegetation index (NDVI), within one year, in mountain forests. The NDVI is widely used to assess forest cover, forest biomass, forest carbon, etc. As such, the quality of an NDVI is directly related to output precision. In steep mountain forests, the issue of the variability of NDVI caused by topography appears not to have attracted much attention. In these regions of high topographic variability, the intra-annual illumination conditions vary significantly from the dynamics of the sun-terrain-sensor geometry. These variances, in turn, affect the spectral response and the derived vegetation indices. The seasonal variations of forest NDVIs in rough terrain are studied in this manuscript. We conducted a statistical analysis of random samplings from May 2013 to October 2014, of all available cloud-free NDVI images of Landsat 8 OLI. We studied how illumination conditions (IL) affect intra-annual NDVI on deciduous and coniferous forests. The findings indicate that IL and NDVI have significant positive linear correlations, and the slope coefficients of linear functions are U-shaped over the course of a year. Meanwhile, we found a positive linear correlation between IL heterogeneity and NDVI variability. Thus, the effects of illumination conditions on NDVI or NDVI-related estimations should be taken into account in both forest monitoring and the quantitative analysis of mountainous areas.

Manuscript IV aims to develop a robust and straightforward haze removal method for optical satellite images. In addition to cloud contamination, multispectral remotely-sensed images are often degraded by haze, which in turn reduces visual interpretability and affects further image analysis. Thus, haze detection and removal techniques are essential to multispectral image preprocessing. We successfully removed the haze from the Landsat 8 OLI data in the project Lin4Carbon area. We also found an improved method to achieve better performance in very high spatial resolution images, which we describe in this manuscript. Unlike 
the existing haze thickness map-based (HTM-based ) method, the proposed method estimates the HTM from the blue band's mean vector L2-norm for each pixel, using a given window size. Also, we improved the compensation strategies for both haze and haze-free pixels. The proposed method has been successfully applied to a variety of very high-resolution optical satellite imagery with complex haze coverage in densely built-up areas.

Manuscript $\mathbf{V}$ aims to reconstruct a higher spatial resolution multispectral image from a so-called "pan-weighted multispectral reconstruction" approach. To some extent, multispectral data is one of the more critical data sources for various index and index-based forest variable estimation, forest type classification, etc. compared to panchromatic images. Interpolation and pansharpening methods are commonly used to improve the spatial resolution of multispectral data. However, the disadvantages of these methods are also evident, in that traditional interpolation methods result in loss of spatial detail, and pansharpening methods tend to suffer from spectrum loss of fidelity. Thus, we attempt to reconstruct higher spatial resolution multispectral images through a method involving joint panchromatic and multispectral images. The proposed method is characterized by the fact that the reconstructed multispectral image pixels inherit the spatial details of the neighboring pixels of the panchromatic image. Through quantitative analysis and visual comparison, it shows that the proposed method produces better performance than traditional interpolation methods. Manuscript V also demonstrates two potential applications, namely refined pansharpening and NDVI calculation. This method would be an alternative or improved upsampled interpolation method, which may become a widely accepted technique used to refine and preprocess satellite images.

Overall, this thesis attempts to solve several of the technical problems encountered in the implementation of the Lin4Carbon project on optical satellite image processing. The data availability and application potential of optical satellite images are often limited by bottlenecks, including their susceptibility to variations from the atmosphere, terrain and illumination conditions. Once these bottlenecks are overcome, optical remote sensing will be more likely to be widely used in forest monitoring. All of the methods presented in this thesis are concerned with the refinement of image pre-processing techniques, which are in turn expected to have a wide range of applications and are applicable to various optical satellite images. 


\section{Acknowledgments}

First of all, I would like to express my deepest gratitude to my supervisor, Prof. Dr. Christoph Kleinn, for his careful guidance, encouragement, and continuous support. I am impressed and influenced by his rigorous scientific attitude, knowledge, and enthusiasm.

I would also like to thank Prof. Yuanchang Lu and his team from Chinese Academy of Forestry (CAF) for their support in this project's implementation in China and for the financial support provided for a portion of the fieldwork.

Special thanks go to our senior experts and colleagues from our institute, Dr. Hans Fuchs, Dr. Lutz Fehrmann, Dr. Haijun Yang, Dr. Xiaolu Tang, Dr. Nils Nölke, and Dr. Paul Magdon, for their support of and advice in my scientific research. I also express my special thanks to Ms. Sabine Schreiner, for her excellent coordination of my project and continued support, even with my trivial matters. Prof. Dr. Christian Ammer, Dr.Torsten Vor and Dr. Na Lin from Silviculture and Forest Ecology of the Temperate Zones, also provided me significant support.

Sincere thanks to Director Anguo Fan, Mr. Bailing Ding, and Miss Yue'e Chu from Shitai Forest Bureau, Mr. Xiaozhu Wang and Mr. Hongbing Ruan from Jitan Town, and foresters from a number of forest stations.

I would like to thank Master's degree students, Ms. Ping Wu and Ms. Ningxing Lu (Anhui Agriculture University), Ms. Cunyan Wu (CAF), Ms. Julia Böning, Mr. Metodi Panev and Mr. Ezequiel Fiorese (Georg-August-Universität Göttingen), for their assistance with fieldwork and data collection.

This work was supported by the German Federal Ministry of Education and Research (Lin4Carbon project, Grant: 033L049C) and CAF (Lin2Value-CAFYBB2012013).

I have to thank my parents, parents-in-law and younger brothers for their full understanding and continuous support. Here, I would like to especially thank my dear wife, Guozhen Zhang, for her selfless love and encouragement to support my study in Göttingen, Germany.

Göttingen, Germany, July 2018

Dengkui Mo 


\section{Introduction}

\subsection{Background}

Forest is the dominant terrestrial ecosystem on earth (Pan et al., 2013). Forests currently cover 4.03 billion hectares or approximately $31 \%$ of the world's land surface (FAO., 2010). Forest ecosystems not only provide forest products (such as timber and fuel) for economic and social development and people's livelihoods, but forests also have multiple ecological functions (such as air purification, soil and water conservation, and biodiversity conservation). Forests also provide social services for human recreation and entertainment. In addition, forests play an essential role in the global carbon cycle (Pan et al., 2011) and help mitigate climate change, because they act as "carbon sinks", absorbing atmospheric carbon and storing it within forest biomass and soil. Conversely, harvested and degraded forests are significant sources of greenhouse gas (GHG) emissions (FAO., 2013). As degradation reduces forest benefits, we expect forests to sustain their high productivity, high carbon adsorption, resistance to natural disturbances, and the maintenance of forest ecosystem services, which are also the objectives of sustainable forest management (SFM).

In order to achieve the sustainability of forest resource utilization, accurate forest information must be provided as support for forest management decisions. This essential, accurate information is obtained through forest monitoring, which involves the collection of data on both the quality and quantity of forest resources (in terms of status and change). This information, in turn, is required for forest planning, operating and formulating adaptive forestry policies (Lei et al., 2009). In addition to the inherent complexities of forests, such as the composition of tree species, age classes and stand structures, forests are also widely distributed, geographically remote and often poorly accessible. These factors make the design and development of efficient forest monitoring systems necessary.

Terrestrial forest inventory is at the very core of forest monitoring, whether for small-scale, enterprise-level forest management inventory (FMI), or large-scale, national forest inventory (NFI). Terrestrial forest inventory mainly consists of such tasks as sampling design, plot design, field data collection, statistical analysis, and reporting. Of all monitoring tasks, field data collection is the most challenging, because it is the most labor-intensive, time-consuming and 
costly. In order to accomplish the task of field data collection, a large number of field plots must be visited, and from several to dozens of forest variables must be measured or investigated. Also, even though a terrestrial forest inventory can estimate quantitative and qualitative attributes of a forest at the plot level, it is a challenge to accurately regionalize those areas that have not been visited and surveyed.

For an operational and efficient forest monitoring system, forest inventories with integrated, remotely sensed data have been proposed as the means to optimize sampling and plot design, in order to reduce costs. This has made it possible to regionalize estimated forest target variables into maps. In the last two decades, integrated remote sensing forest inventories have been widely adopted by the U.S. Department of Agriculture (Smith, 2002), European NFIs (McRoberts and Tomppo, 2007), and the UN-REDD program (Asner et al., 2013). The advantages of forest monitoring based on field observations and remote sensing data have been widely recognized and validated. Recently, Wallner et al. (2018) examined the use of remotely sensed data for pre-stratification and sample plot allocation in the sampling design stage of forest inventory within a very mixed forest. Wallner was able to show that the reduction of sampling plots leads to a cost saving of $21 \%$.

A variety of aerial or satellite remote sensing data sources (such as optical images, synthetic aperture radar (SAR), light detection and ranging (LiDAR) and terrestrial laser scanning (TLS)) have been widely used in forest monitoring. Considering the characteristics of availability, affordability, broad coverage, spatial resolution and revisit frequency, optical satellite images have quite apparent advantages and are indispensable in the implementation of forest monitoring. However, optical satellite images are easily affected by many factors (such as weather situations, topographic relief, and illumination conditions), all of which directly lead to the distortion of image spectra and spatial displacement and further limit the performance of forest monitoring. Although a variety of general optical image processing techniques have been proposed, refined image processing techniques for forest monitoring are relatively inadequate. Additional detailed image processing issues also need to be studied, such as how intra-annual illumination conditions affect the consistency and stability of forest monitoring results when using multitemporal optical satellite data in mountain forests, as well as how to solve the spatial scale mismatch issue between field plot observations and the pixels of optical satellite images. Therefore, this thesis will concentrate on further development of image processing technologies 
that may overcome these issues, with the aim of making optical satellite images more available and adaptable to forest monitoring.

\subsection{Structure of the manuscripts in this thesis}

The initial overall objective of our study is to develop an operational forest carbon monitoring approach with integrated remote sensing and field surveys. In the Lin4Carbon project, we implemented in Chinese mountain forests, the forest carbon monitoring framework we designed is shown in Figure 1. The left side of the framework mainly shows the image processing steps and techniques of optical satellite data. The right side of the framework is mainly the traditional field inventory used to complete the required forest data collection and estimations.

During the implementation of the Lin4Carbon project, we found that specific challenges regarding optical satellite image processing techniques (from the atmosphere, rugged mountains to field plots) still should be addressed. However, there is still a lack of commonly accepted and adopted approaches. Thus, this cumulative thesis is intended to contribute a number of refined image processing approaches, in order to enhance the adaptability and performance of optical satellite images in a forest monitoring framework.

The themes of all five manuscripts are problem-oriented and are specifically related to image processing approaches. The specific reasons for initiating these topics and research are detailed in the "Research Questions section".

The research objectives of the five manuscripts are briefly described as follows:

(1) To develop a simple and straightforward topographic normalization model for optical satellite images using moving, window-based rotation-correction in mountain forests (Manuscript I).

(2) Using a local parameter estimation method, to establish a general, topographic normalization approach for optical satellite images under conditions of rough terrain. Moreover, this method's performance in forest type classifications was further verified (Manuscript II). 
(3) To evaluate the effects of intra-annual illumination conditions on NDVI time series in mountainous forests, with a view to understanding the mechanism of illumination conditions on forest biomass and carbon estimates (Manuscript III).

(4) To develop a robust and straightforward haze removal method for optical satellite images, with a view to improving the availability of optical satellite data (Manuscript IV).

(5) To reconstruct a higher spatial resolution multispectral image from a so-called "panweighted multispectral reconstruction" approach, using synchronously acquired panchromatic images as a reference, thus making the pixel area of multispectral data closer to the area of the field plot (Manuscript V).

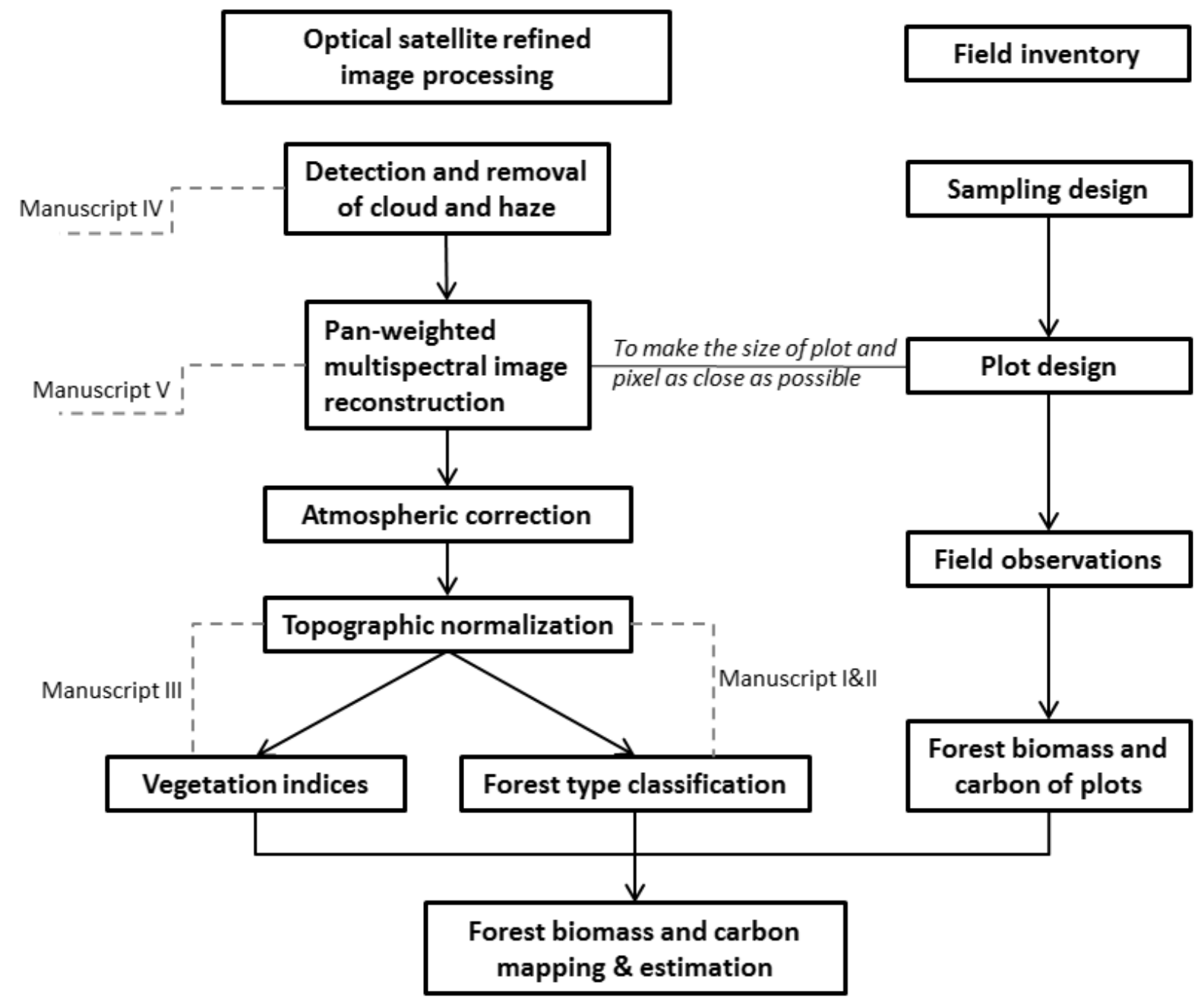

Figure 1. The framework of forest carbon monitoring techniques based on optical satellite imagery and field observations, and the five selected manuscripts that attempt to solve the challenges encountered during the implementation of the Lin4Carbon project. 
To avoid our studies being limited to just case studies, we expect that all innovative and optimized image processing techniques should be versatile, operational, and deployable in any forest monitoring systems. The connected manuscripts constitute almost a complete optical satellite image processing system. Problems related to the atmosphere, rough mountains, multitemporal images, as well as the scale-matching issue between field plot and pixel (see Figure 1) are all covered. Therefore, we can integrate all manuscripts into this synthetic thesis (see Synthesis section). In addition, these proposed approaches can be applied to more general forest monitoring and are not limited to forest carbon monitoring only. 


\subsection{A brief introduction of forest monitoring}

\subsection{Clarification of forest monitoring or forest inventory}

It is worth first clarifying what is forest monitoring before introducing it. Activities that systematically collect forest information are often referred to as "forest inventory" (Bechtold and Patterson, 2005; Hyyppä et al., 2008) or "forest monitoring” (Banskota et al., 2014; Jucker et al., 2017). Forest inventory is usually organized by project and is a data collection activity that helps generate the required forest information within a given area and duration. Forest monitoring is typically used in a broader sense, and refers to forest inventories that can observe and assess the status quo and changes of forests over the long-term and in a repeated manner (Kleinn, 2015). In addition, in most cases, when all or part of the data source is remote sensing data, the forest inventory is called "forest monitoring" (QuangBao and Hoa, 2016; Romijn et al., 2015). Thus, in this thesis, "forest inventory" refers specifically to "traditional or terrestrial forest inventory" that rely on field surveys and statistics (sampling and modeling) to collect and estimate forest information. Moreover, "forest monitoring" refers specifically to those forest monitoring and mapping activities that integrate remote sensing data.

For decades, remote sensing data has been used to inventory forest resources. In fact, except in military sectors, forestry departments were among the first to use remote sensing data across large spatial extents in order to support their forest monitoring tasks (Koch, 2013). With the development of remote sensing techniques, forest monitoring projects widely use various remote sensing data collected from a variety of active and passive sensors on terrestrial, aircraft or satellite platforms.

\subsection{Exploring forest monitoring applications using remote sensing data}

To illustrate the role of remote sensing data in forest monitoring, a brief review of recent studies may be helpful. Here, forest mapping and estimation of forest aboveground biomass and carbon are of interest.

\section{Mapping of forest and forest change}

Quantifying the distribution and changes of forests in spatial extent is considered to be virtually the most direct and essential application of remote sensing data in forestry. Potapov et 
al. (2008) used Landsat Thematic Mapper (TM) and Enhanced Thematic Mapper Plus (ETM+) images to produce the world's forest landscape maps, which support the development of a general global strategy for nature conservation and serve as a baseline for monitoring deforestation and forest degradation. Hansen et al. (2013) produced the Global Forest Change dataset, in which they analyzed 654,178 growing seasons Landsat 7 ETM+ scenes under a cloudbased platform called Google Earth Engine and created global forest loss and gain maps with a spatial resolution of 30 meters. Although some scientists have commented that the Global Forest Change dataset cannot distinguish between tropical forests and plantations, or even herb crops, which has caused severe underestimation of forest loss (Tropek et al., 2014), it still demonstrates the advantages of remote sensing data in forest mapping and change monitoring. Besides the above mentioned regional or global scale studies, more studies have focused on algorithmic or methodological optimizations to improve their performance in forest mapping using small-scale data from optical data or hybrid data (integration of optical data and radar or LiDAR data) (Hakkenberg et al., 2018; Joshi et al., 2016; Zylshal et al., 2016). Optical remote sensing data is indispensable for forest mapping and change monitoring at local, regional and global scales.

\section{Estimating forest aboveground biomass and carbon}

Remote sensing-based methods for estimating forest aboveground biomass (AGB) and aboveground carbon (AGC) have expanded in recent decades to include a variety of modern methods. Many of these methods that estimate AGB can be used for AGC estimation, particularly in forests where AGB is often used as a proxy for AGC (Hulvey et al., 2013a). Therefore, we do not explicitly emphasize the difference in methods of AGB and AGC estimation using remote sensing throughout this section.

Detailed and accurate spatial biomass reference data is required for AGB estimation. In order to construct reference data, species-specific allometric models based on direct field measurements of tree attributes such as $\mathrm{DBH}$, tree height and wood density need to be developed. The second methods is to use large plot-level datasets from regional and national forest inventories to build biomass conversion models (Lu et al., 2016). Although time-consuming and laborious, collecting vast AGB reference data at the plot level is indispensable, and allometric models and biomass conversion models established from these data should be evaluated. 
AGB estimation based on remote sensing also needs to establish models (bridges) between AGB estimates from plot surveys and derived pixel variables from the remote sensing data. Before building these models, plots should be co-registered with resolution and location of the pixels from given remote sensing data. Using such established models and derived variables images from remote sensing data, pixel-wise AGB maps can predict the type of covering of an area for an entire remote sensing dataset.

Recently, several studies have focused on selecting derived variables from different remote sensing data sources and optimizing modeling, provided case studies for estimating forest AGB or AGC using optical, active remote sensing data, or integrated multisource data. Han et al. (2013) utilized multi-temporal Landsat images to estimate AGC of the Moso bamboo forest between 1986 and 2008 in Anji County, China. Fuchs et al. (2009) and Lumbres and Lee (2014) used the k-nearest neighbor (k-NN) technique to estimate AGC using combined multi-spectral satellite images and sample-based field inventory data. Asner et al. (2012) studied the use of minimal field data to facilitate rapid LiDAR calibration to generate carbon maps in tropical forests. Morel et al. (2011) used a data combination of field and ALOS PALSAR to quantify the carbon stock and geographical distribution of forest and timber plantations in Sabah, Malaysia. Tian et al. (2012) used two different methods (nonparametric and parametric) to explore the AGB estimation in the cold and arid regions of northwestern China, relying on field data and three different remote sensing data (dual-polarized ALOS PALSAR, airborne LiDAR data, and SPOT-5 images). Saatchi et al. (2011) suggested that the fusion of LiDAR or InSAR derived forest height with the polarimetric backscatter can provide an improved estimation of AGB. In addition, due to constraints such as cost and large area operability, studies on terrestrial laser scanning (TLS) in forest AGB and AGC estimation are not discussed.

\subsection{Challenges}

Although significant progress has been made in the use of remote sensing data in earth observations over the past several decades, advances in remote sensing technologies also require

time to reach a operational capacity and accuracy for monitoring and reporting in the case of forest cover and related biomass (carbon stocks) and their changes over time. 
Efficient collection of ground truth data is the first challenge to overcome, especially for large-scale forest monitoring. It is almost impossible to collect ground-survey data concurrently with remote-sensing data acquisition over a large area. Another option would be to use airborne or satellite LiDAR to measure and estimate forest information, such as tree height, stand density, volume, etc., to statistically or mechanically build various forest biomass or carbon models, or to use on-site measurements and other optical remote sensing observations (Goetz and Dubayah, 2011). It is a challenge and almost impossible to use LiDAR in large-scale (thousands to millions of square kilometers), full coverage forest monitoring due to cost, logistics and enormous data volume. It is considered to be cost-effective and operational when using footprints of airborne and spaceborne LiDAR as stratified sampling tools to measure and monitor forest resources (Wulder et al., 2012). However, it is still worth determining whether or not LiDAR can achieve the accuracy of field inventory and replace traditional field surveys. Richardson and Moskal (2011) found that the density of shorter trees (tree height less than 20 meters) was significantly underestimated when using airborne LiDAR, and suggested continuing to study alternative remote sensing techniques improve aerial LiDAR for assessing the understory structure. In the coming years, efficient ground truth data acquisition will continue to be a challenge, and it is expected that advances in remote sensing technologies will make it possible to replace traditional methods.

Another major challenge is remote sensing data processing technologies to enable the operation and implementation of forest monitoring. A comprehensive review of all aspects involved in data processing techniques from various sensors is beyond the scope of this thesis, and we are only concerned with optical satellite data processing techniques here. Many features of optical satellite imagery such as high spatial resolution, high revisit frequency, global coverage, data availability and cost-effectiveness make them an indispensable component in forest monitoring systems. More importantly, optical satellite imagery can be used to extrapolate to larger spatial domains from measurements or estimates of field inventory and LiDAR (Guo et al., 2010).

Before the use of optical satellite data, various considerations related to the quality and integrity must be taken into account, including the geometric and radiation characteristics of the image. Various geometric correction techniques based on specific sensors, digital elevation models and high-precision ground control points have been standardized and integrated into 
various remote sensing image processing systems. Geometric correction is a relatively mature technology and there have been few relevant, innovative studies in the past decade. Image radiation is easily affected by the atmosphere between the ground and sensor. Because atmospheric scattering, absorption and emission effects on the received signal of the optical sensors vary with the wavelength, wavelength-specific radiation correction procedures are required (Wulder, 1998). The removal of atmospheric influences is a crucial pre-processing step for analyzing surface reflectance images. Compared to topographic corrections, atmospheric correction techniques are more difficult than topographic corrections, possibly because the variations are more severe and complicated to directly quantify in the atmosphere than that with topography. When optical images are obscured by thick clouds, thin clouds or haze, thick cloud detection and removal are first needed, and then if necessary, degraded images should be restored through detection, removal and compensation of thin clouds and haze. The high frequency and broad coverage of clouds and haze result in degraded optical images and low availability. The effects of clouds and haze in different optical bands are band-specific, and they are easily confused with large bright ground objects and are not readily distinguishable in highspatial resolution images. Thus, the development of a series of standardized techniques to handle the degradation of optical images from atmospheric and severe weather conditions remains a challenge of the field.

In mountain forests, it is also difficult to eliminate the effects of terrain and illumination conditions on the spectral and radiation characteristics of optical images. Spectral and radiation distortions reduce the accuracy of forest mapping and forest variables estimation, and to a certain extent lead to the need for more field plots. Also, because a large number of forests are located in mountainous areas, it is necessary to use topographic normalization to eliminate distortions caused by topography and illumination on the optical images of mountain forests. Due to the different angles of incident illumination, the difference in terrain orientation often results in variations in signal values between pixels with similar forest types and stand structure characteristics (Soenen et al., 2005). Also, the degree of variability of these signal values is band-specific and related to the type and structural characteristics of the forest. Therefore, in order to eliminate spectral and radiative distortions from optical images, the illumination image and map of forest type need to be accurately simulated (although often impossible to accurately obtain in advance) to build hierarchical models between pixel values and illumination conditions 
on different wavelength bands. The lack of a priori forest type data and accurate illumination images with the same spatial resolution as the remote sensing image constrains the quality of the constructed model. Moreover, several topographic normalization models based on global optimization parameters are prone to overcorrection, which may be due to high variations in forest landscapes and illuminations at large scales. Therefore, efficient and adaptive topographic normalization preprocessing is crucial although difficult for mountain forest monitoring.

Further, various vegetation indices, such as the proxy of plant activity, biomass, and cover, can be used to assess forest dynamics (Verbesselt et al., 2016) and their uncertainty analysis limits the accuracy of forest monitoring. For example, forest stands of the same type and structural characteristics have different vegetation index values under different illumination conditions, which results in inaccurate estimates of forest biomass or carbon. Moreover, it is difficult to answer which image should be selected from a series of vegetation index images to produce forest biomass or carbon maps, and provide objective criteria for this selection. Relevant studies have produced contradictory conclusions (Adhikari et al., 2016; Galvão et al., 2016; Valeriano et al., 2016). Also, there is a lack of vegetation index products that take into account the effects of illumination conditions in public. Uncertainty analysis of the illumination conditions for the estimation of forest variables derived from vegetation indices is very important for accurate forest monitoring in mountain forests and should be emphasized and further studied.

Finally, field plots and coregistered pixels are combined to establish and validate forest biomass models. Once the target variables (regarding forest biomass) from field observations of plots are estimated from field observations, potential variables of pixels that matched the field plots both spatially and dimensionally are selected for the remote sensing-based forest biomass models. There are many types of potential variables, such as spectral images, transformed images, vegetation indices, and textures. However, It is still difficult to determine the variables that are most appropriate for modeling biomass estimation ( $\mathrm{Lu}$ et al., 2016). Meanwhile, the issues that underly matching pixels and plots are often overlooked, but they are also the source of errors within forest biomass modeling, especially when the plot is smaller than the pixel in size or when the plots fall in fragmented forests. When the plot is smaller than the pixel in size, it is a forest biomass modeling problem based on sub-pixel variables, or a problem of mixed pixel decomposition. Thus, in constructing forest biomass models based on remote sensing data, the 
selection of potential variables from remote sensing data and scale matching of field plots and pixels should be analyzed and thoroughly processed.

In summary, the above topics are the real challenges that may be encountered in an operational forest monitoring program using optical satellite images, including effective ground truth acquisition, atmospheric correction, terrain correction and scale matching issues. Once "operational" in the forest monitoring system is emphasized, it is natural to consider costs and conquer the technical problems encountered. These challenges cannot be treated individually, and we should take into account the interplay between the proposed solutions to ensure that these technologies can be seamlessly integrated into various operational forest monitoring systems. Of course, issues are not limited to the above technical challenges, but also include forest monitoring under various definitions of "Forest", multi-source data fusion, uncertainty analysis, understanding of the capabilities and limitations of remote sensing-based forest monitoring, etc. Currently, the acquisition of various optical satellite data becomes accessible and affordable. It is expected that innovative and advanced image processing technologies will help the vast amount of archived optical satellite data be widely used for rapid and accurate forest monitoring. 


\section{Background of Forest Carbon Monitoring in China}

\subsection{Forest carbon and sustainable forest management}

An increase in forest carbon stocks has become an important research goal for government organizations and the scientific community to help mitigate global climate change. According to the Intergovernmental Panel on Climate Change (IPCC), reducing deforestation is the forestry mitigation measure with the most significant and immediate effects on atmospheric $\mathrm{CO}_{2}$ concentration (Metz and Davidson, 2007). The Reducing Emissions from Deforestation and Forest Degradation (REDD) program was launched to create a financial value for storing carbon in forests and encourage developing countries to reduce deforestation and investing in lowcarbon pathways for sustainable development (http://www.un-redd.org). Initially, the nature of REDD rejected requests for participation from countries that already had either high or low deforestation rates (Birdsey et al., 2013). Then, "REDD+", as a climate change mitigation mechanism, was developed by Parties to the United Nations Framework Convention on Climate Change (UNFCCC). The "+" in REDD + refers to the conservation and enhancement of forest carbon through sustainable management of forests. Thus, the mechanism of REDD+ is to encourage developing countries to maintain their forests status and enhance forest carbon stocks of participating countries through improved sustainable forest management and conservation. Those participating countries would receive payments for their results-based actions of reducing forest carbon emissions.

\subsection{Forest biomass/carbon monitoring in China}

In order to implement REDD+ in China, like in any participating countries, the baselines for long-term forest monitoring at regional or national levels must be established. Surveying and reporting of accurate and reliable forest carbon information are critical to the implementation of project REDD+. Thus an efficient forest carbon monitoring system needs to be developed.

In China, where forests are widely distributed and diverse, it is a challenge to obtain comprehensive and accurate data on forest biomass and carbon. The three-level forest inventory system in China consists of NFI (level I), the forest management planning inventory (FMPI, level II) and the forest operational design inventory (level III) (Lei et al., 2009). However, these 
forest inventory systems are not specifically designed for REDD +, which is also typical in other regions. and it is difficult to fully meet REDD +'s forest monitoring requirements due to their different inventory objectives, technical standards and outputs. While these forest inventory data can be used as ancillary data for forest carbon monitoring, their data quality and conversion models need to be validated in advance. In addition, a third-party independent forest carbon monitoring and evaluation system needs to be developed for REDD+ because of its international project status. Because carbon stock models rely on data from terrestrial inventories and remote sensing can cover large geographical areas and also remote and hard-to-reach areas, an efficient forest carbon monitoring requires the integration of field surveys and remote sensing. In China, forest carbon monitoring of the project REDD+ is mainly aimed at plantation forests.

\subsection{Status of plantation forests in China}

With vast afforestation activities in the past decades, China has the largest afforested area in the world, expanding from 62 million hectares in 2008 to 69.33 million hectares in 2014 . Plantation forest occupies $36 \%$ of the forest land area, from which $46 \%$ timber volume was harvested (data reported in Chinese from 8th NFI (2009-2013), available at national and provincial levels: http://211.167.243.162:8085/8/index.html).

The Chinese government has launched several projects to convert croplands and marginal lands into forests, to reduce soil erosion and water quality degradation (Peng et al., 2014). One successful project is the Sloping Lands Conversion Program (SLCP), where farmers are provided with grain and cash subsidies in exchange for converting farmland on steep slopes into forests and grasslands (Li et al., 2011). During 2001-2010, SLCP led to the conversion of millions of hectares of marginal croplands to forests across 25 provinces; meanwhile, it involved 124 million people and cost the government 233 billion Chinese Yuan. Plantation area of China has been continually growing under such country-driven afforestation projects.

However, the quality of these plantations did not synchronously improve with quantity. Most of the plantations in China are monocultures and short-rotation timber-oriented forests. These forests are more fragile under threats such as forest fires, pests and extreme weather events. Extreme ice storms destroyed 20 million hectares of forest in Central and South China in 2008, most of which were pure forest plantations and bamboo forests which have been intensively 
reforested since the 1980s (Zhou et al. (2011). The ice storm also caused secondary damage, such as soil erosion, landslides, insects and disease attacks and more frequent forest fires.

In general, mixed forests have increased forest biomass and forest carbon stocks compared with monocultures. He et al. (2013) and Hulvey et al. (2013b) found that mixed-species stands obtain higher forest biomass and carbon than monoculture stands. Fan et al. (2013) suggested that the transformation of conifer monoculture into mixed conifer-hardwood plantations would be one forest management strategy that would increase ecosystem carbon sequestration. There is a huge potential for carbon forestry in China if we switch to more appropriate forest management strategies in forest plantations. 


\section{Overview of the Lin4Carbon project}

China's plantation forests are the largest in the world in terms of area (Carle et al., 2002) and their area has continued to increase under the China's afforestation program in the past 30 years (Shilong et al., 2015). It is a challenge to monitor such a large area of afforestation and assess the potential of converting them into sustainable carbon forests.

Reliable estimates of forest carbon stocks are the basis for implementing market mechanisms within the framework of actual climate policies, such as REDD + . The fact that specific forest carbon monitoring planning and implementation standards have not yet been established may be mainly due to different national conditions, so there is no forest carbon monitoring guidance applicable to all countries. Mountain forests are widely distributed and scattered, with various age classes and stand structures, which make forest carbon monitoring difficult in China.

China intends to introduce the concept and operation of multi-functional "near nature forestry" from Germany to improve the quality of plantation forests regarding tree species complexity, stock volume, and natural disturbance resistance, and realize the transformation of plantation forests into sustainable carbon forests. Germany has a long history and rich experience in the sustainable management of forests. Since the 1980s, the concept of "near nature forestry" has been widely recognized by the public in Germany (Bauhus et al., 2013). It is the original intention of the Lin4Carbon project to achieve the sustainability of China's plantation development by combining the actual needs of China and the knowledge of sustainable forest management from Germany.

The Lin4Carbon project focuses on forest carbon monitoring and optimizes forest management strategies to achieve sustainable forests and increased carbon sequestration in forests. Lin4Carbon is also committed to promoting the sustainable development of the SinoGerman forest science and technology cooperation and dialogue. The study site is a typical mountainous forest area in southern China, characterized by steep slopes (see Figure 2) and high forest coverage, and dominated by monoculture plantation and species-rich natural forest (see Figure 3). 
The overall objectives of the Lin4Carbon project are to develop innovative and practical techniques and methodologies for improved forest monitoring and forest land use. Specifically, the project wants to optimize forest structure and management strategies of monoculture plantations in China by introducing the concept of advanced "multifunctional forests" or "nearnatural forests" and silvicultural technologies to achieve increased timber productivity, carbon sequestration and long-term sustainability of forests. This part of the task is mainly implemented by Dr. Xiaolu Tang from the project team (see his thesis for details (Tang, 2015)). The project also strives to develop operational forest carbon monitoring systems using remote sensing imagery and fieldwork data, which is the primary goal of this thesis.

Within the framework of the Lin4Carbon project, a forest carbon monitoring system which integrates field inventories and optical remote sensing has been already established. Field inventories were carried out to provide the ground truth of land cover and plot-level forest estimates of volume, biomass and carbon stocks. During the inventories, in addition to collecting core variables such as tree species, tree height, $\mathrm{DBH}$, and stand density, additional information on biodiversity and site conditions was recorded. Field inventories of three different scales were established as follows:

(1) Land use inventory (systematic grid $3 \times 3 \mathrm{~km}$, cover the whole extent of the study site) collected the ground truth of land cover, which was used to establish the interpretation key of remote sensing data for land use type classification;

(2) Forest management inventory (systematic grid of $500 \times 500 \mathrm{~m}$ ) selected from given forest sub-compartments;

(3) Stand inventory (systematic grid of $100 \times 100 \mathrm{~m}$ ) from selected forest stand.

In the two years of fieldwork, in addition to completing the above-mentioned inventory tasks, we also produced discs of the main coniferous and broad-leaved species in the study area and conducted laboratory analysis. These data were used to construct growth models and estimate biomass and carbon for each tree species.

This study was funded mainly by the German Federal Ministry of Education and Research (Bundesministerium für Bildung und Forschung, BMBF) (Lin4Carbon project, Grant: 033L049C) and the Chinese Academy of Forestry (Grant No. Lin2Value-CAFYBB2012013). These projects 
were implemented in Shitai County, Anhui Province, the People's Republic of China. It is worth mentioning that the Deutsche Forschungsgemeinschaft (DFG) project (Research Group FOR2432, implemented in India) also provided Worldview 3 test images for one of the manuscripts focusing on haze removal of very high-resolution optical satellite images.

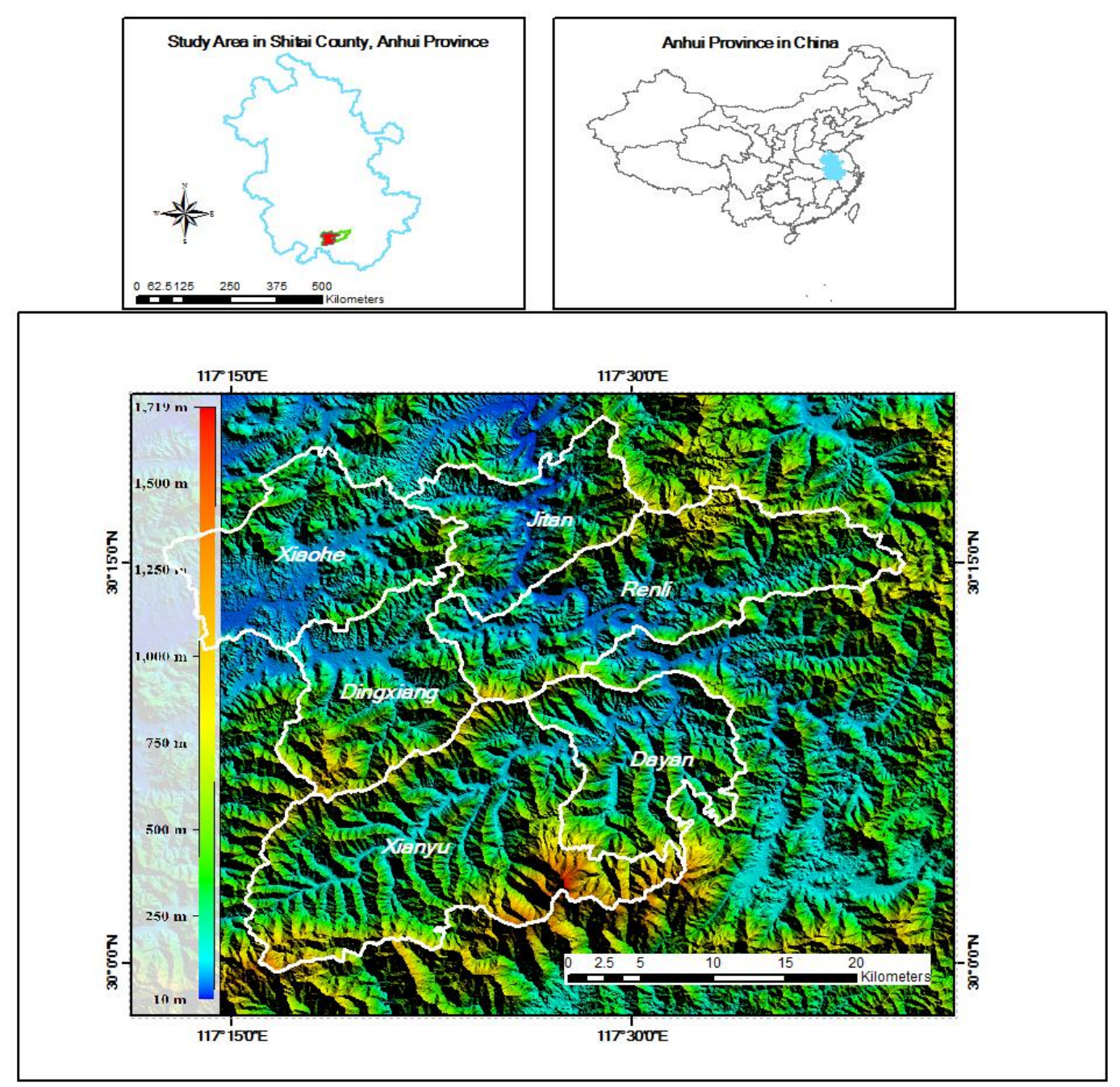

Figure 2. Location of study site and illustration of terrain conditions produced from SRTM 1 Arc-Second Global simulated as shaded relief with sun elevation $=60^{\circ}$, and sun azimuth $=45^{\circ}$. 


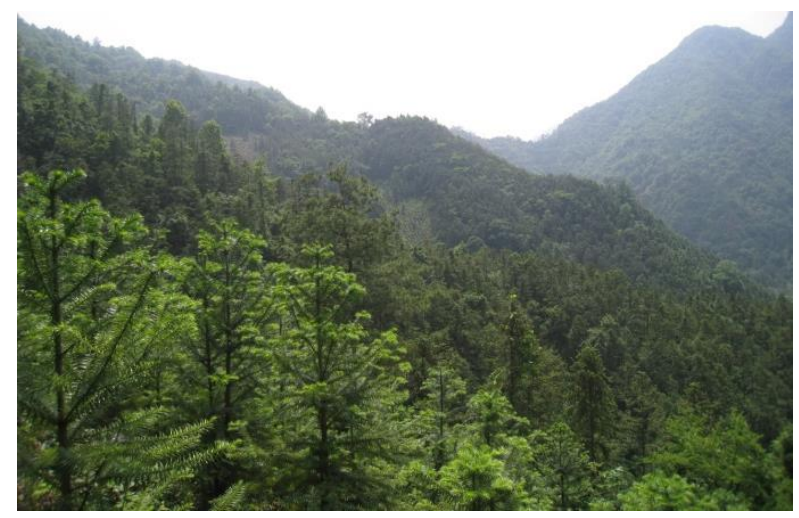

(1)

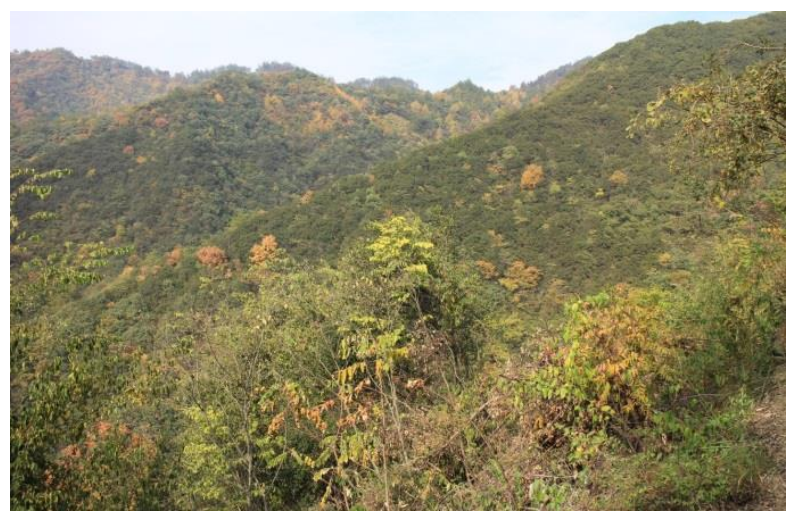

(2)

Figure 3. (1) Chinese fir (Cunninghamia lanceolate) plantation and (2) mixed natural forest from the study site. 


\section{Research Questions}

\subsection{Question-oriented research}

In order to develop highly operational forest carbon monitoring systems for mountain forests, this research is question/challenge oriented. This thesis confronts a series of difficulties encountered during the implementation of Lin4Carbon project and attempts to develop corresponding techniques for optical satellite image processing.

\subsection{How to eliminate the spectral distortion caused by the variability of illumination conditions in mountain forests?}

Research related to this topic can be found in Manuscripts I and II

Reducing or eliminating spectral or radiation distortions has always been a challenge for the classification of forest types or extraction of forest information from optical satellite imagery (Avitabile et al., 2012; Vanonckelen et al., 2015). In mountain forests, even the same forest cover shows spectral variability under different illumination conditions (Figure 4). Topography and instantaneous solar parameters determine illumination conditions. Once the optical satellite imagery has been acquired, the image is highly visually consistent with the corresponding illumination conditions in terms of light-dark variation (Figure 5). Illumination conditions can, therefore, be applied to the topographic correction or normalization of optical remote sensing images.

Most wavelength-dependent topographic normalization models and their variants such as C-Correction (Hantson and Chuvieco, 2011; Riaño et al., 2003), Minnaert (Ge et al., 2008; Richter et al., 2009) and Rotation correction models (Tan et al., 2013) outperform wavelengthindependent ones such as the Cosine model (Civco, 1989). The performance of such models heavily depends on the quality of the estimation of empirical parameters between spectral data and illumination data. Because global estimation methods show clear overcorrection in practice, we attempted to estimate local parameters from local samples for topographic normalization. The following sub-questions should be addressed:

(1) Which wavelength-dependent topographic normalization model should be selected? 
(2) How to determine the size of the local window?

(3) Does the window size affect the heterogeneity of intra-class spectra?

(4) Does the proposed method improve classification accuracy with a small number of training samples?

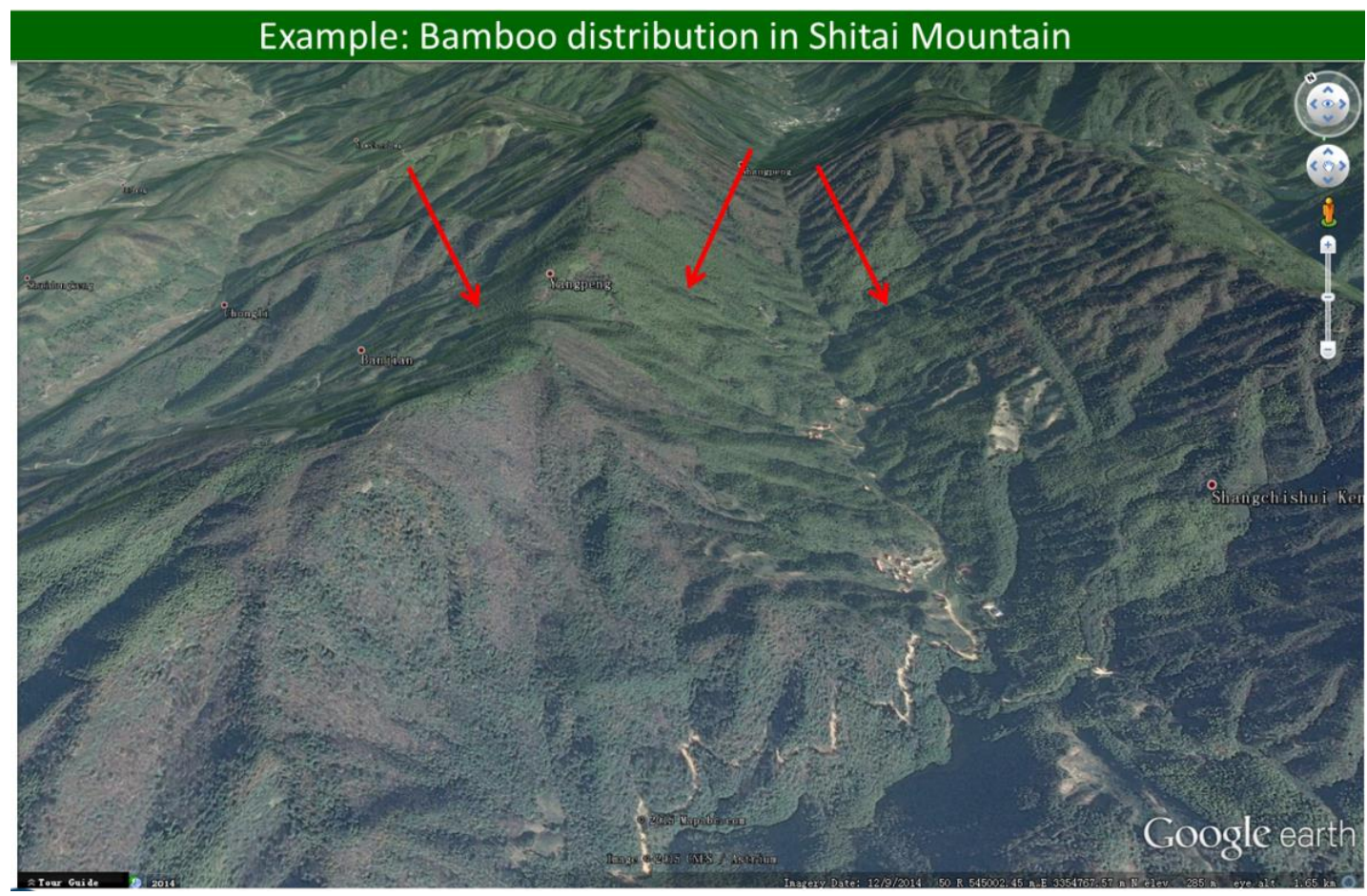

Figure 4. Due to the variation of topography and illumination conditions, even the same type of land use has a variety of spectral characteristics. The pixels pointed by the red arrows are pure bamboo forests under different terrain and illumination conditions (image from Google Earth). 


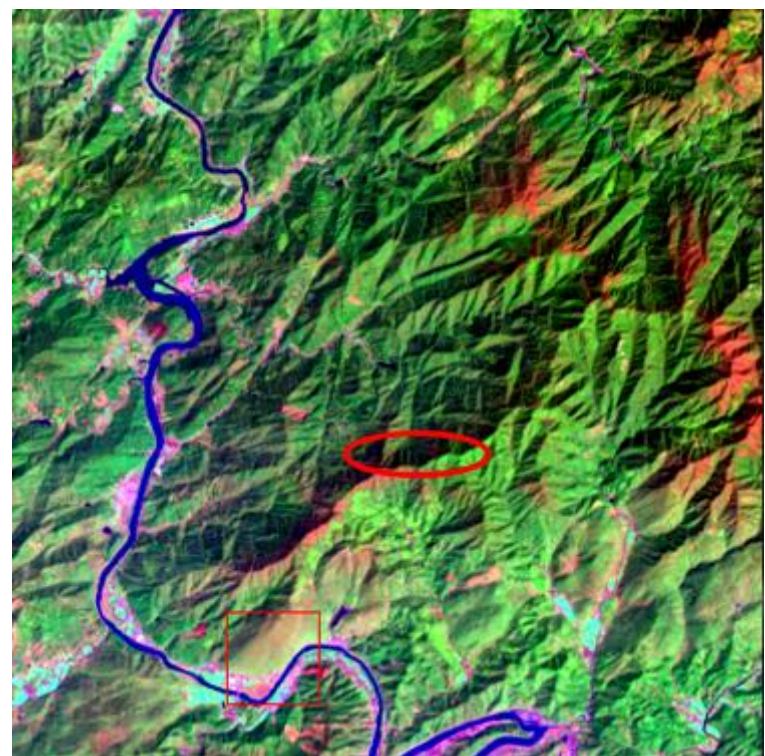

(1)

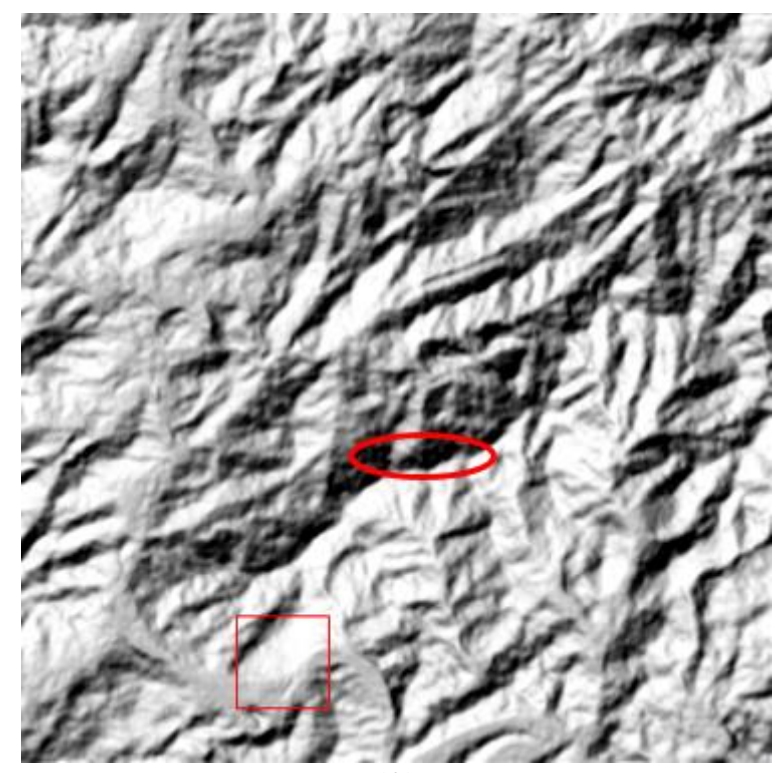

(2)

Figure 5. The image is highly visually consistent with the corresponding illumination conditions regarding light-dark variation. (1) Original Landsat 8 (Band composition $=$ Band 7, 5, 3, using 2\% linear stretch), (2) Illumination condition (IL). Note: Date acquired =2014-03-30, Sun azimuth = $135.84^{\circ}$, Sun elevation $=55.65^{\circ}$ and DEM using SRTM 1 Arc-Second Global elevation data.

\subsection{What is the NDVI response mechanism during illumination conditions during the year?}

Research related to this topic can be found in Manuscript III

NDVI is undoubtedly the most widely used optical satellite imagery products for forest and vegetation monitoring (Ke et al., 2015). The NDVI products can be considered as core variables for assessing forest-related target variables such as forest carbon stocks (Tan et al., 2007), forest biomass (Riihimäki et al., 2017), and forest type mapping. However, it is not yet fully understood which NDVI product periods are best for estimating forest-related target variables and how illumination conditions affect the accuracy of these mountain forest estimates. We assume that, besides being affected by the phenological characteristics of vegetation itself, NDVI may also be affected by illumination conditions, especially in mountain forests. In order to understand the effects mechanism of the illumination conditions on NDVIs, the NDVI time 
series throughout the year were collected, and synchronized illumination conditions were simulated. The following sub-questions are of interest and should be solved.

(1) What are the effects of illumination conditions within the NDVI time series during the year for evergreen or deciduous forests?

(2) Does the heterogeneity of illumination conditions affect the variability of NDVI?

(3) For a given illumination condition, can the heterogeneity of illumination conditions be used in advance to assess the variability of NDVI or NDVI-related forest target variables?

\subsection{How to remove haze from optical satellite images to enhance data availability?}

Research related to this topic can be found in Manuscript IV

Cloud and haze coverage severely limits the availability of optical satellite imagery. For cloud-covered optical satellite imagery, cloud pixels can be detected and then masked (Zhu and Woodcock, 2012, 2014), and there is little room to recover the cloud-covered pixels. However, for haze-covered optical satellite imagery, there is still potential to recover the haze-affected pixels. Haze removal helps to improve visual interpretability and allow for further image analysis for optical satellite image preprocessing. We have implemented an algorithm for removing haze from Landsat 8 images based on the literature (Makarau et al., 2014b; Makarau et al., 2016) and the results are excellent as shown in Figure 5. However, performance is limited when applied to a variety of VHR (very high spatial resolution) optical satellite images with complex haze cover over densely built-up regions.

Thus, it is naturally of interest to develop a general haze removal method for optical satellite images with various spatial resolutions. As a way of distinguishing and creating an innovative method from the methods mentioned above, we are more concerned with the development of haze removal methods for VHR optical satellite imagery. 


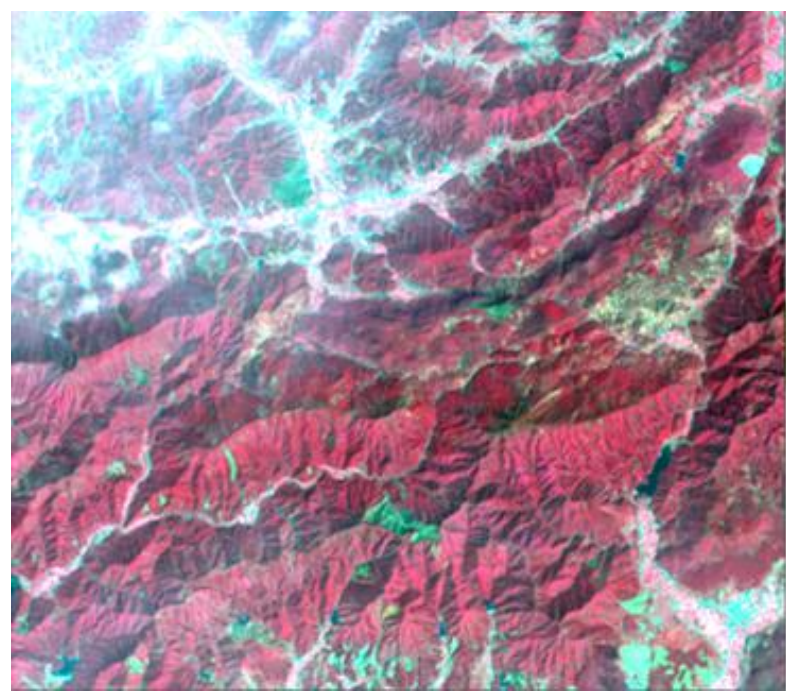

(1)

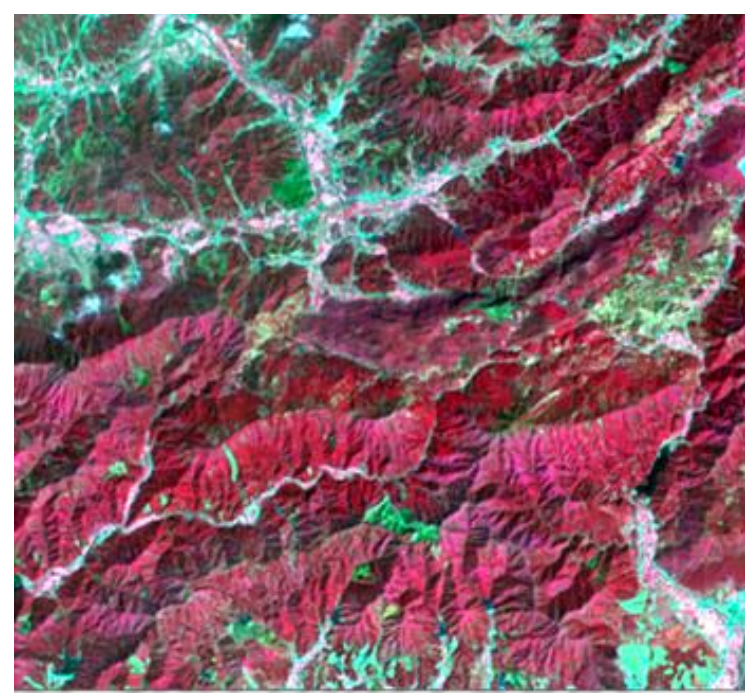

(2)

Figure 6. Example of before and after haze removal of optical satellite images (selected area of study site). (1) Original Landsat 8 (band composition (NIR, red, green), using 2\% linear stretch, (2) haze removed image by our implementation using an algorithm from (Makarau et al., 2014b; Makarau et al., 2016).

\subsection{How to reconstruct high-spatial-resolution multispectral images using the panchromatic image as a reference making the size of field plot and pixel closer?}

Research related to this topic can be found in Manuscript $\mathbf{V}$

It is common in practice that the pixel area of a multispectral optical satellite image is not equal to the area of an inventory plot (as shown in Figure 7). An alternative approach is to resample multispectral image using one of three well-known interpolation methods, such as nearest neighbor, bilinear interpolation or bicubic convolution so that the pixel size equals the size of the inventory plot. However, these interpolation methods cannot help multispectral images to inherit finer spatial details from the ground truth when needing a finer scale resolution. Thus, there is a need for a method to manage the heterogeneity of forests in the inventory plots, which is common when plots are located on land cover borders or in fragmented forests. 

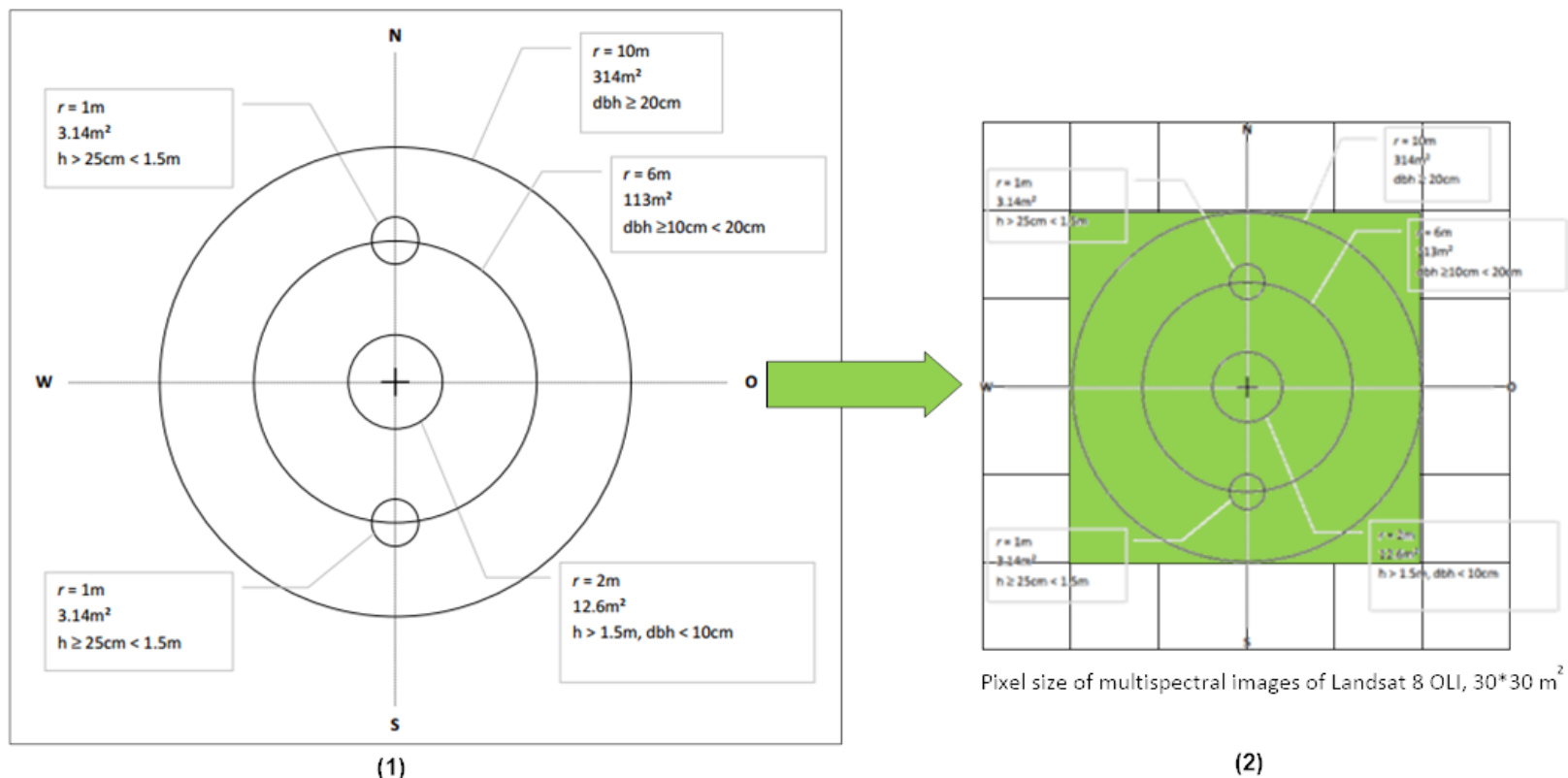

(2)

Figure 7. Inventory plot size does not match the pixel size of the multispectral image. (1) Inventory plot design from Lin4Carbon Forest Inventory Guidelines Field Protocol, Dr. Lutz Fehrmann, August 2013, (2) pixel size of multispectral images of Landsat 8 OLI, $30 * 30 \mathrm{~m}^{2}$.

Because a panchromatic image itself has higher spatial resolution and more spatial details than that of the corresponding multispectral images, the following scientific questions have been proposed naturally:

(1) How to reconstruct multispectral images with higher spatial resolution and inherit the spatial details from the corresponding panchromatic images?

(2) Does the proposed approach have a broader application scenario than its original ground cover? 


\section{Synthesis: Further Developing Processing Techniques of Optical Satellite Images in the Context of Forest Monitoring}

Forests are the most widely distributed types of terrestrial vegetation and play a key role in providing valuable timber products and environmental services to humankind. They also shape and directly affect the dynamic processes of regional and global ecosystems (Banskota et al., 2014; Wulder, 1998). However, due to human and natural disturbances, the distribution, structure, biodiversity, and biomass of forests are being dynamically affected and changed. The status and dynamics of forests and scientific decision-making with regard to forest management depend on accurate and efficient forest monitoring.

Terrestrial forest monitoring systems, which rely solely on sampling and field observations, are able to collect detailed plot-level forest ground truths and to estimate forest target variables. However, these systems also have limitations, such as being time-consuming, costly and with a lack of capacity to accurately regionalize forest data. Various optical satellites have been continuously observing the earth's surface and collecting wide-area forest images with rich spectral information. These medium and high spatial resolution optical satellite data include open-source data, such as Landsat (Griffiths et al., 2014; Zhu et al., 2012), Sentinel 2 series data (Drusch et al., 2012), and commercial data, such as SPOT, Pléiades and Worldview series data (Poli et al., 2015; Souza Jr et al., 2003). Without relying on detailed forest plot observations, monitoring forests by means of optical remote sensing has been widely used to observe forest cover changes (Hansen et al., 2013) and for rough forest type mapping. However, this method is ineffective in estimating forest biomass and forest carbon. Similarly, obtaining more detailed forest variables (such as tree height, $\mathrm{DBH}$, tree density, and biodiversity) by optical remote sensing alone is difficult. Thus, it is necessary to develop monitoring designs that combine field observations and remote sensing (Tomppo et al., 2008).

Integrated optical satellite imagery and field observation techniques are considered to be operational and have been widely used for forest monitoring on local, regional and even global scales (Boisvenue et al., 2016; Chen et al., 2017; Chirici et al., 2016; Reese et al., 2003). However, forest monitoring techniques have not yet been standardized and are not uniformly accepted, mainly due to complex or uncontrollable conditions, such as the atmosphere (Mannschatz et al., 2014), topography, illumination (Tokola et al., 2001), data availability and 
scale-matching issues (Magdon and Kleinn, 2013; Marceau and Hay, 1999). For these so-called disturbances or uncertainties, more refined and specific image processing techniques must be developed for optical satellite images.

\subsection{Refined image processing techniques for severe atmospheric conditions}

Severe atmospheric variations (such as clouds and haze) are common challenges to effective optical satellite image processing, especially with regard to monitoring terrestrial resources. In practice, complete clear-sky optical satellite data is relatively scarce. The availability of optical satellite data is undoubtedly affected by certain atmospheric conditions (such as clouds, cloud shadow, and haze), which also naturally limit the timeliness of remote sensing applications. Meanwhile, the coverage of both cloud and haze will reduce the robustness of the estimation of geophysical properties (Hong and Zhang, 2018), will cause errors in land cover classification (Ying and Guindon, 2003) and will also change detection capabilities (Qiu et al., 2017).

Recently, many cloud detection approaches have been developed for optical satellite images (Hagolle et al., 2010; Latry et al., 2007; Panem et al., 2005; Zhu et al., 2015; Zhu and Woodcock, 2014). Among these approaches, the Fmask (Function of mask) algorithm and its variants have exhibited moderately better performance in terms of operational cloud detection than have other algorithms. The Fmask is an open-source algorithm and has been successfully integrated into the processing of Landsat Level-1 data products (Foga et al., 2017). Typically, the pixels covered by clouds and cloud shadows are detected first and then removed by a mask, but recovering these pixels are difficult. In practice, although large-area haze-covered pixels can easily be mistakenly detected as cloud pixels, haze-contaminated pixels have the potential to be restored (see Figure 8).

Haze not only affects the blue band or shorter wavelength band but also further affects the longer wavelength band. Haze can lead to various difficulties, such as visual interpretation, image classification, and the calculation of vegetation indices and estimation of forest variables. However, most state-of-the-art approaches focus on de-hazing methods using terrestrial outdoor photos (He et al., 2011) or Google Earth RGB screenshots (Long et al., 2014). These methods 
cannot be directly applied to the haze removal of raw data from optical satellite images, due to their differing assumptions. Some studies on the removal of haze from optical satellite images have been proposed. These studies have been based on different perspectives, such as wavelet analysis (Du et al., 2002), haze optimized transformation (HOT) (Jiang et al., 2016), haze thickness map (HTM) (Makarau et al., 2014a; Makarau et al., 2016), and other approaches (Liu et al., 2017). Haze removal methods based on HTM were developed and integrated into commercial remote sensing software, such as ACTOR 2/3 (Makarau et al., 2014b; Makarau et al., 2016), PCI Geomatics and ERDAS IMAGINE. These are considered to be state-of-the-art haze removal modules for optical satellite images. After testing various data, we found that HTM methods perform well on most optical satellite data. However, these methods face challenges in cases of very high spatial resolution (VHR) satellite images, particularly in cases where large bright objects can be confused with haze.

Manuscript IV presents a robust and straightforward haze removal method for VHR satellite imagery. The method is based on the assumption that haze in satellite images is locally uniform and can be detected from the background value and large bright objects. We proposed the L2-norm HTM (see Manuscript IV for more details) of the blue band, in order to avoid under or overestimation of HTM. In addition, new compensation strategies for haze pixels have been developed. Experiments on several VHR optical satellite images at different sites, including Worldview 3, Pléiades and Gaofen-2 images, validated the effectiveness of the proposed method in terms of haze removal rate, haze removal before and after the spectral consistency, etc. The method can also be applied to medium and low spatial resolution optical satellite images. The refined haze removal method helps to enhance the method's adaptability to various optical satellite data. Nevertheless, we can hardly conclude that the proposed method has absolute advantages over other methods, due to the lack of widely accepted evaluation indicators. Rather, we just use this method as an alternative haze removal method for VHR optical satellite images. In the future, we recommend developing novel haze removal evaluation indicators and to apply the proposed method to various applications, in order to indirectly evaluate the method's performance. 


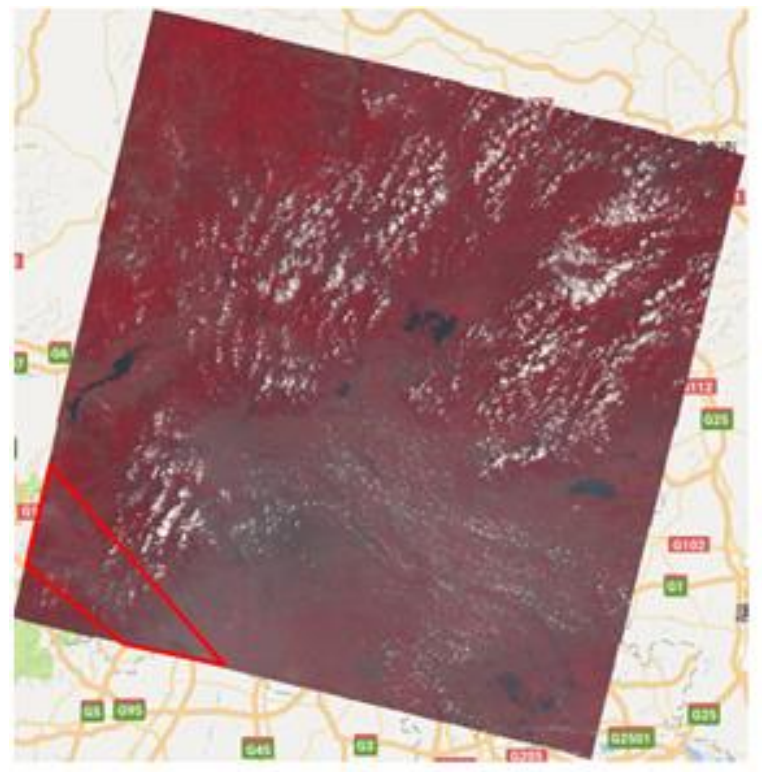

(1)

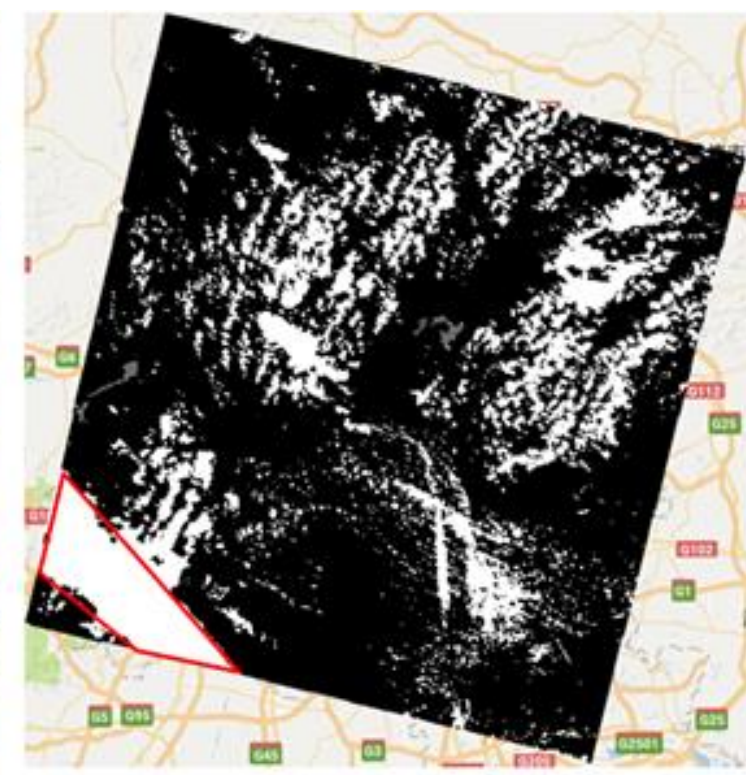

(2)

Figure 8. The availability of optical satellite images is restricted by strong atmospheric variability, such as clouds, haze, etc. Compared to the difficulty of restoring cloud pixels, hazecovered pixels have the potential to be restored. In practice, haze-covered pixels are sometimes incorrectly detected as cloud pixels (see the area marked by the red polyline). (1) Original Landsat 8 false-color combination, (2) cloud detection for Landsat 8 is based on Fmask (Zhu et al., 2015). LANDSAT_SCENE_ID = "LC81230322016189LGN00".

\subsection{Refined image processing techniques for rugged}

\section{mountainous terrain}

In rugged mountainous terrain, in addition to severe atmospheric conditions (such as clouds and haze), the effects of illumination, which are determined by the combination of topography and solar radiation, have a significant impact on optical satellite images. Also, it is worth emphasizing that most forests in mountainous areas are covered by different atmospheric conditions. Therefore, it is crucial to eliminate the effects of illumination conditions on optical images used for mountain forest monitoring. Topographic normalization (or correction) models have been recognized as having the ability to compensate for the high variability in pixels caused by different illumination conditions (Riaño et al., 2003). 
Spectral variation or distortion due to the variability of illumination conditions naturally leads to difficulties and inaccuracies in applications, such as image classification, change detection, and inversion of biophysical parameters. Rugged terrain and solar irradiation parameters jointly determine illumination conditions. In mountain forests, differences in illumination conditions result in significant differences in the radiation or reflectance of the pixels between the shaded and illuminated areas, even for the same forest types (as shown in Figure 9). These conditions cause further difficulties in forest monitoring applications, such as the classification of forest types, calculation of vegetation indices, and estimation of forest biomass.

Of all the various proposed topographic normalization models, wavelength-dependent models and their variants (such as C-Correction (Hantson and Chuvieco, 2011; Riaño et al., 2003), Minnaert (Ge et al., 2008; Richter et al., 2009) and rotation correction models (Tan et al., 2013)) outperform wavelength-independent models, such as the Cosine model (Civco, 1989). The performance of wavelength-dependent models depends heavily on the quality of the model's empirical parameter estimates between spectral data and illumination data. When using global parameter estimation methods (as most studies have already done), the performance of these models is acceptable, but only when applied to small pieces of data. However, in practice, when the global estimation methods are applied to large-area optical satellite data or data with more diverse types of land cover, their results usually show a clear tendency for overcorrection.

In order to avoid the "overcorrection" mentioned above, the concept of local parameter estimation was introduced. In addition, moving window or local parameter estimation-based rotation correction models were developed and tested for Landsat 8 OLI images in Manuscripts I and II. In terms of intra-class spectral consistency, forest type classification, and visual evaluation, the presented method performs better and is more robust for use with different window sizes, compared to the topographic normalization model based on global parameter estimation. In addition, the proposed method does not require any prior land cover information. The proposed method's simplicity and robustness demonstrate its potential as a standard preprocessing method for optical satellite imagery, especially for areas of rugged mountainous terrain. 


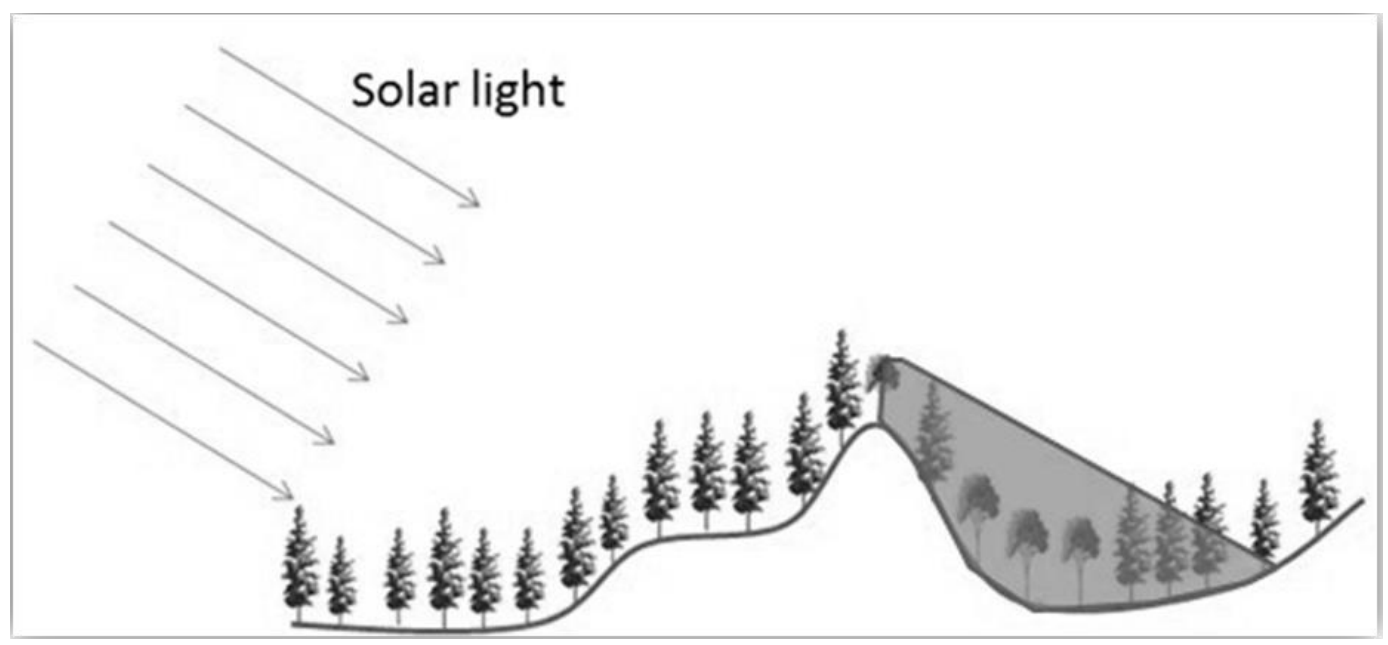

Figure 9. Effect of illumination conditions caused by topography and solar light on reflectance. The reflectance or radiation in the shaded areas of the mountain forest is relatively low, while the effect in the irradiated area is the opposite.

Usually, topographic normalization models refer to the topographic correction of a singlephase optical satellite image using corresponding instantaneous illumination conditions. However, a few studies have focused on the effects of topographic normalization on the time series of optical satellite images. Interestingly, when different models are applied to different study sites, their conclusions are contrary with regard to whether or not topographic normalization is really needed for optical remote sensing time series data. The case studies are as follows:

Adhikari et al. (2016) carried out C-Correction for Landsat seasonal time series between June 2012, and October 2013. The study concluded that NDVI and RSR (reduced simple ratio) were not affected by topographical effects in the tropical mountain landscapes throughout the year. Moreira et al. (2016) concluded that topographic correction was required for a consistent reduction of the topographic effect on the vegetation indices, including NDVI from rugged terrain when the C-Correction method (Moreira and Valeriano, 2014) was used. Chance et al. (2016) assessed the impact of topographic correction both with and without the sun-canopysensor correction algorithm (Fan et al., 2014). Chance indicated that topographic correction does not cause a substantial difference in the quality of forest change detection. In the review of forest monitoring using Landsat time series, Banskota et al. (2014) emphasized that topographic normalization is necessary for the inter-comparison of images in the time series. Having virtually 
no doubt regarding the role of topographic normalization, Mack et al. (2017) directly introduced topographic normalization into their workflow for producing land use and land cover maps for Germany, using Landsat time series and Lucas (Land Use/Cover Area frame Survey) in-situ data.

From the studies mentioned above, it is difficult to conclude whether or not topographic normalization is necessary for optical satellite image time series, and which model can be more widely accepted. It is possible to conclude that each topographic normalization model has its own specific assumption. Each model is also possible only applicable to specific site conditions. However, the effects of terrain illumination on spectral radiation and reflectivity are notable and cannot be ignored when monitoring mountain forests. Thus, Manuscript III attempts to directly study the interaction mechanism of NDVI and illumination conditions during the period of a year, while ignoring the specific topographic normalization model, using Landsat 8 OLI time series. Regardless of whether the area has an evergreen or deciduous forest, there is a significant linear positive correlation between each pair of illumination conditions and NDVI. Also, the slope coefficients of these linear functions exhibit a U-shaped feature over the period of a year. Meanwhile, the heterogeneity of illumination conditions and the variability of NDVI were also observed to be positively linear related. This, at least as a case study, highlights the effects of illumination conditions on NDVI or that NDVI-related estimations should be taken into account in mountain forests.

\subsection{Refined image processing techniques for scaling issues}

Almost all scientific observations or measurements are scale-related. Forest monitoring also needs to address scaling issues, specifically regarding temporal and spatial scales. Over the past few decades, these scale issues have increasingly attracted scientists' attention in different fields, such as spatial scale effects on landscape patterns (Turner et al., 1989), hydrological modeling (Blöschl, 1999; Blöschl and Sivapalan, 1995), and on remote sensing (Marceau and Hay, 1999). In forestry, (Hwang et al., 2011) produced real-time vegetation dynamics from downscaled multi-temporal satellite images, and Magdon and Kleinn (2013) studied the uncertainties of forest area estimates caused by the scale effects of the minimum crown cover criterion. 
In the context of forest monitoring combined with field observations and optical remote sensing data, scale issues may come into play in many forms, such as spatial resolution (pixel size) and spatial extent of remotely sensed data, the size of sampling plots, the frequency of data acquisition, and scale effects within sampling designs. Of all these, the most likely scale issue would be the mismatch in size between sample plots and image pixels, especially when using medium and coarse spatial resolution remote sensing data in forest monitoring on a regional or global scale (Lu et al., 2016; Wang and Gertner, 2013; Wang et al., 2009). Taking into account survey costs, total survey area, travel distance and accessibility, field plot may be designed to be smaller than the pixel of the remote sensing image in size. However, this can cause some plots to be located in mixed pixels or on pixel boundaries. This scale mismatch can cause further uncertainty in pixel-wise forest estimation and mapping. Even though some spectral unmixing techniques have been applied in enhanced forest cover mapping (Gudex-Cross et al., 2017) and forest aboveground biomass monitoring (Lu et al., 2005), uncertainties caused by scale mismatch have not been completely eliminated. Moreover, these new techniques can only produce forest inventory maps with the spatial resolution of multispectral or hyperspectral images.

In order to eliminate the above-mentioned effect of scale mismatch, we assume that the closer the size between sample plots and image pixels, the more the degree of uncertainty can be reduced for forest target variable estimates and mapping. Manuscript V presents a general approach to enhancing the spatial resolution of multispectral images using the panchromatic images captured synchronously. As a result of the synchronous acquisition, panchromatic and multispectral images are very well, jointly registered, with uniform atmospheric and illumination conditions. This naturally makes the panchromatic images a source of high-quality reference data for the reconstruction of higher spatial resolution multispectral images. The proposed approach is inspired by the bilateral filter algorithm (Elad, 2002), which has been widely used in the fields of image processing and machine vision. However, few studies have been applied to the reconstruction or unmixing of satellite optical multi-spectral images. For each band of resized multispectral images, our approach replaces the intensity of each pixel with a weighted average of intensity value from adjacent pixels, within a given window. Each weighted matrix is derived from the element-wise multiplication between a panchromatic image intensity matrix and a given two-dimensional Gaussian matrix (for more details of the algorithm see Manuscript V). The experimental results show that the reconstructed multispectral images can preserve the spectral 
characteristics of the original multispectral images, as well as the spatial details of the panchromatic images. In addition, two applications are also tested (such as the refinement of image fusion and NDVI). The superior performance of our proposed method may indicate that it has the potential to address scale mismatch issues frequently encountered in forest monitoring, at least to some extent.

\subsection{Concluding remarks}

Forest monitoring requires the collection of rapid, accurate and spatially detailed forest information, with the aims of reporting forest statuses and trends, as well as supporting applications pertaining to scientific forest policy, planning, and sustainable management. Forest monitoring can be extended to a range of spatial extents, from small community forests to national and even global forests. Considering operability, cost, and efficiency issues, integrated field inventory and remote sensing forest monitoring is widely accepted and applied. When building a realistic forest monitoring system, in addition to the sampling design and modeling related to field inventory, a series of image processing techniques related to remote sensing should also be developed and optimized, in order to meet specific forest monitoring conditions. Although state-of-the-art instruments (such as airborne and terrestrial laser scanning equipment) have been successfully applied in the field of forest monitoring, their cost and scanning area are not suitable for the very large areas of forest monitoring required, especially when compared to optical satellite data. Therefore, in this thesis, which is only concerned with optical satellite images, we highlighted the effects of severe atmosphere conditions and the rugged terrain, and we developed refined image processing techniques to eliminate these effects.

First of all, there is a need to calibrate the spectral or radiative distortions caused by variations of atmospheric conditions. The downward and upward radiation from the earth's surface can be modified by the atmosphere, especially by severe atmospheric conditions, such as clouds and haze. As a consequence, the digital numbers recorded in the pixels of optical images may not truly represent the reflection characteristics of the observed ground objects. Even more significantly, optical images contaminated by clouds and haze may be thrown away and become useless. Some studies have been conducted with a view to removing the effects of these severe atmospheric conditions and improving the availability of remote sensing data. Compared to 
cloud-covered pixels, previous studies have shown that detecting and removing haze-covered pixels from optical satellite images is even more difficult. As shown in Manuscript IV, a welldesigned haze removal method includes the refinement of haze detection, haze removal and compensation. Such a method also helps to enhance the visual interpretation of and preserve the consistency of the spectral characteristics of the observed ground objects. In addition, the application of the proposed approach to various optical remote sensing data has been demonstrated.

Besides taking into account severe atmospheric conditions, the topographic effects caused by the variations in illumination conditions should not be neglected. The illumination condition image is jointly determined by the topography and the solar parameters (the same as instantaneous solar parameters when acquiring a remote sensing image). This means that a remote sensing image has a unique corresponding illumination condition image. The time series of remote sensing images also have corresponding time series of illumination condition images. Thus, for mountain forests, selecting an appropriate topographic normalization model to eliminate the spectral or radiative distortion caused by the variation of illumination conditions is indispensable (see Manuscripts I and II). Meanwhile, it is also necessary to understand the intraannual interaction mechanism between spectral images/vegetation indices and illumination conditions. This is vital for uncertainty analysis in forest monitoring (see Manuscript III).

Also, before regionalizing the results of a field inventory, the mismatch issue between field plot size and pixel size should be considered. This mismatch issue may lead to inaccurate modeling between field inventory data and remote sensing data, and may further lead to inaccurate maps, especially in fragmented forests. In fact, not only the size, but also the shape, orientation, and type of the field plot should be well designed in advance, so that the field plots can be matched with the corresponding remote sensing image pixels. For the size mismatch issue, Manuscript V presents a generalized technique that can be used to reconstruct a higher spatial resolution of multispectral images by using simultaneously acquired panchromatic images as a reference.

This thesis (under the general, technical framework of integrated field inventory and optical remote sensing for forest monitoring) has developed and optimized several key image processing techniques. The aim of these techniques is to mitigate the effects of severe 
atmospheric conditions, illumination conditions and the size of field plots. Combined with other effective and widely accepted image processing techniques, the approaches presented and investigated in this thesis may help to build a complete chain of optical satellite image processing techniques for the monitoring of mountain forests. All the proposed techniques are demonstrated, and their potential applications should not only be limited to forest monitoring. Although the performance of the proposed techniques is positive and convincing from the perspective of our study, the performance of the synthesis of these proposed techniques for forest monitoring should be further tested and evaluated.

Looking ahead, forest monitoring based on field inventory and optical remote sensing will continue to play an essential role in assessing the detailed status and dynamics of forests. However, this monitoring should be conducted in a more efficient, accurate and informative manner. More refined image processing techniques (as described in this thesis) need to be developed or introduced and validated for use in complex forest monitoring conditions. It is also worth emphasizing that many image processing techniques are interrelated in the forest monitoring technical framework and that their synergies should also be considered and evaluated.

\section{References}

Adhikari, H., Heiskanen, J., Maeda, E.E., \& Pellikka, P.K. (2016). The effect of topographic normalization on fractional tree cover mapping in tropical mountains: An assessment based on seasonal Landsat time series. International Journal of Applied Earth Observation and Geoinformation, 52, 20-31

Asner, G.P., Mascaro, J., Anderson, C., Knapp, D.E., Martin, R.E., Kennedy-Bowdoin, T., van Breugel, M., Davies, S., Hall, J.S., Muller-Landau, H.C., Potvin, C., Sousa, W., Wright, J., \& Bermingham, E. (2013). High-fidelity national carbon mapping for resource management and REDD+. Carbon Balance and Management, 8, 7

Asner, G.P., Mascaro, J., Muller-Landau, H.C., Vieilledent, G., Vaudry, R., Rasamoelina, M., Hall, J.S., \& van Breugel, M. (2012). A universal airborne LiDAR approach for tropical forest carbon mapping. Oecologia, 168, 1147-1160

Avitabile, V., Baccini, A., Friedl, M.A., \& Schmullius, C. (2012). Capabilities and limitations of Landsat and land cover data for aboveground woody biomass estimation of Uganda. Remote Sensing of Environment, 117, 366-380

Banskota, A., Kayastha, N., Falkowski, M.J., Wulder, M.A., Froese, R.E., \& White, J.C. (2014). Forest monitoring using Landsat time series data: A review. Canadian Journal of Remote Sensing, 40, 362-384 
Bauhus, J., Puettmann, K., \& Kühne, C. (2013). Close-to-nature forest management in Europe: does it support complexity and adaptability of forest ecosystems. In (pp. 187-213): Routledge, The Earthscan Forest Library

Bechtold, W.A., \& Patterson, P.L. (2005). The enhanced forest inventory and analysis program: national sampling design and estimation procedures. US Department of Agriculture Forest Service, Southern Research Station Asheville, North Carolina

Birdsey, R., Angeles-Perez, G., Kurz, W.A., Lister, A., Olguin, M., Pan, Y.D., Wayson, C., Wilson, B., \& Johnson, K. (2013). Approaches to monitoring changes in carbon stocks for REDD. Carbon Management, 4, 519-537

Blöschl, G. (1999). Scaling issues in snow hydrology. Hydrological Processes, 13, 2149-2175

Blöschl, G., \& Sivapalan, M. (1995). Scale issues in hydrological modelling: a review. Hydrological Processes, 9, 251-290

Boisvenue, C., Smiley, B.P., White, J.C., Kurz, W.A., \& Wulder, M.A. (2016). Integration of Landsat time series and field plots for forest productivity estimates in decision support models. Forest Ecology and Management, 376, 284-297

Carle, J., Vuorinen, P., \& Del Lungo, A. (2002). Status and trends in global forest plantation development. Forest Products Journal, 52, 12-23

Chance, C.M., Hermosilla, T., Coops, N.C., Wulder, M.A., \& White, J.C. (2016). Effect of topographic correction on forest change detection using spectral trend analysis of Landsat pixelbased composites. International Journal of Applied Earth Observation and Geoinformation, 44, 186-194

Chen, B., Xiao, X., Li, X., Pan, L., Doughty, R., Ma, J., Dong, J., Qin, Y., Zhao, B., \& Wu, Z. (2017). A mangrove forest map of China in 2015: Analysis of time series Landsat 7/8 and Sentinel-1A imagery in Google Earth Engine cloud computing platform. Isprs Journal Of Photogrammetry and Remote Sensing, 131, 104-120

Chirici, G., Mura, M., McInerney, D., Py, N., Tomppo, E.O., Waser, L.T., Travaglini, D., \& McRoberts, R.E. (2016). A meta-analysis and review of the literature on the k-Nearest Neighbors technique for forestry applications that use remotely sensed data. Remote Sensing of Environment, 176, 282-294

Civco, D.L. (1989). Topographic normalization of Landsat Thematic Mapper digital imagery. Photogrammetric Engineering and Remote Sensing, 55, 1303-1309

Drusch, M., Del Bello, U., Carlier, S., Colin, O., Fernandez, V., Gascon, F., Hoersch, B., Isola, C., Laberinti, P., \& Martimort, P. (2012). Sentinel-2: ESA's optical high-resolution mission for GMES operational services. Remote Sensing of Environment, 120, 25-36

Du, Y., Guindon, B., \& Cihlar, J. (2002). Haze detection and removal in high resolution satellite image with wavelet analysis. IEEE Transactions On Geoscience and Remote Sensing, 40, 210217

Elad, M. (2002). On the origin of the bilateral filter and ways to improve it. IEEE Transactions On Image Processing, 11, 1141-1151

Fan, H.B., Liu, W.F., Wu, J.P., Li, Y.Y., Yuan, Y.H., Liao, Y.C., Huang, R.Z., \& Su, X.Q. (2013). Ecosystem Carbon Pools in Mixed Stands of Hardwood Species and Masson Pine. Journal of Tropical Forest Science, 25, 154-165

Fan, Y., Koukal, T., \& Weisberg, P.J. (2014). A sun-crown-sensor model and adapted Ccorrection logic for topographic correction of high resolution forest imagery. Isprs Journal Of Photogrammetry and Remote Sensing, 96, 94-105

FAO. (2010). Global Forest Resources Assessment 2010 Main Report. FAO 
FAO. (2013). Climate change guidelines for forest managers. FAO

Foga, S., Scaramuzza, P.L., Guo, S., Zhu, Z., Dilley, R.D., Beckmann, T., Schmidt, G.L., Dwyer, J.L., Joseph Hughes, M., \& Laue, B. (2017). Cloud detection algorithm comparison and validation for operational Landsat data products. Remote Sensing of Environment, 194, 379-390 Fuchs, H., Magdon, P., Kleinn, C., \& Flessa, H. (2009). Estimating aboveground carbon in a catchment of the Siberian forest tundra: Combining satellite imagery and field inventory. Remote Sensing of Environment, 113, 518-531

Galvão, L.S., Breunig, F.M., Teles, T.S., Gaida, W., \& Balbinot, R. (2016). Investigation of terrain illumination effects on vegetation indices and VI-derived phenological metrics in subtropical deciduous forests. GIScience \& Remote Sensing, 53, 360-381

Ge, H., Lu, D., He, S., Xu, A., Zhou, G., \& Du, H. (2008). Pixel-based Minnaert correction method for reducing topographic effects on a Landsat 7 ETM+ image. Photogrammetric Engineering \& Remote Sensing, 74, 1343-1350

Goetz, S., \& Dubayah, R. (2011). Advances in remote sensing technology and implications for measuring and monitoring forest carbon stocks and change. Carbon Management, 2, 231-244

Griffiths, P., Kuemmerle, T., Baumann, M., Radeloff, V.C., Abrudan, I.V., Lieskovsky, J., Munteanu, C., Ostapowicz, K., \& Hostert, P. (2014). Forest disturbances, forest recovery, and changes in forest types across the Carpathian ecoregion from 1985 to 2010 based on Landsat image composites. Remote Sensing of Environment, 151, 72-88

Gudex-Cross, D., Pontius, J., \& Adams, A. (2017). Enhanced forest cover mapping using spectral unmixing and object-based classification of multi-temporal Landsat imagery. Remote Sensing of Environment, 196, 193-204

Guo, Z., Chi, H., \& Sun, G. (2010). Estimating forest aboveground biomass using HJ-1 Satellite CCD and ICESat GLAS waveform data. Science China Earth Sciences, 53, 16-25

Hagolle, O., Huc, M., Pascual, D.V., \& Dedieu, G. (2010). A multi-temporal method for cloud detection, applied to FORMOSAT-2, VEN $\mu$ S, LANDSAT and SENTINEL-2 images. Remote Sensing of Environment, 114, 1747-1755

Hakkenberg, C., Peet, R., Urban, D., \& Song, C. (2018). Modeling plant composition as community continua in a forest landscape with LiDAR and hyperspectral remote sensing. Ecological Applications, 28, 177-190

Han, N., Du, H.Q., Zhou, G.M., Xu, X.J., Cui, R.R., \& Gu, C.Y. (2013). Spatiotemporal heterogeneity of Moso bamboo aboveground carbon storage with Landsat Thematic Mapper images: a case study from Anji County, China. International Journal of Remote Sensing, 34, 4917-4932

Hansen, M.C., Potapov, P.V., Moore, R., Hancher, M., Turubanova, S., Tyukavina, A., Thau, D., Stehman, S., Goetz, S., \& Loveland, T. (2013). High-resolution global maps of 21st-century forest cover change. science, 342, 850-853

Hantson, S., \& Chuvieco, E. (2011). Evaluation of different topographic correction methods for Landsat imagery. International Journal of Applied Earth Observation and Geoinformation, 13, 691-700

He, K., Sun, J., \& Tang, X. (2011). Single image haze removal using dark channel prior. IEEE Transactions On Pattern Analysis and Machine Intelligence, 33, 2341-2353

He, Y.J., Qin, L., Li, Z.Y., Liang, X.Y., Shao, M.X., \& Tan, L. (2013). Carbon storage capacity of monoculture and mixed-species plantations in subtropical China. Forest Ecology and Management, 295, 193-198 
Hong, G., \& Zhang, Y. (2018). Haze removal for new generation optical sensors. International Journal of Remote Sensing, 39, 1491-1509

Hulvey, K.B., Hobbs, R.J., Standish, R.J., Lindenmayer, D.B., Lach, L., \& Perring, M.P. (2013a). Benefits of tree mixes in carbon plantings. Nature climate change, 3, 869

Hulvey, K.B., Hobbs, R.J., Standish, R.J., Lindenmayer, D.B., Lach, L., \& Perring, M.P. (2013b). Benefits of tree mixes in carbon plantings. Nature Climate Change, 3, 869-874

Hwang, T., Song, C., Bolstad, P.V., \& Band, L.E. (2011). Downscaling real-time vegetation dynamics by fusing multi-temporal MODIS and Landsat NDVI in topographically complex terrain. Remote Sensing of Environment, 115, 2499-2512

Hyyppä, J., Hyyppä, H., Leckie, D., Gougeon, F., Yu, X., \& Maltamo, M. (2008). Review of methods of small-footprint airborne laser scanning for extracting forest inventory data in boreal forests. International Journal of Remote Sensing, 29, 1339-1366

Jiang, H., Lu, N., \& Yao, L. (2016). A high-fidelity haze removal method based on HOT for visible remote sensing images. Remote Sensing, 8, 844

Joshi, N., Baumann, M., Ehammer, A., Fensholt, R., Grogan, K., Hostert, P., Jepsen, M.R., Kuemmerle, T., Meyfroidt, P., \& Mitchard, E.T. (2016). A review of the application of optical and radar remote sensing data fusion to land use mapping and monitoring. Remote Sensing, 8, 70 Jucker, T., Caspersen, J., Chave, J., Antin, C., Barbier, N., Bongers, F., Dalponte, M., Ewijk, K.Y., Forrester, D.I., \& Haeni, M. (2017). Allometric equations for integrating remote sensing imagery into forest monitoring programmes. Global Change Biology, 23, 177-190

Ke, Y., Im, J., Lee, J., Gong, H., \& Ryu, Y. (2015). Characteristics of Landsat 8 OLI-derived NDVI by comparison with multiple satellite sensors and in-situ observations. Remote Sensing of Environment, 164, 298-313

Kleinn, C. (2015). Lecture Notes for the Module "Monitoring of Forest Resources", 5th revised edition. In. Göttingen: Georg-August-Universität Göttingen

Koch, B. (2013). Remote Sensing supporting national forest inventories NFA. FAO knowledge reference for national forest assessments, 15

Latry, C., Panem, C., \& Dejean, P. (2007). Cloud detection with SVM technique. In, Geoscience and Remote Sensing Symposium, 2007. IGARSS 2007. IEEE International (pp. 448-451): IEEE

Lei, X., Tang, M., Lu, Y., Hong, L., \& Tian, D. (2009). Forest inventory in China: status and challenges. International Forestry Review, 11, 52-63

Li, J., Feldman, M.W., Li, S.Z., \& Daily, G.C. (2011). Rural household income and inequality under the Sloping Land Conversion Program in western China. Proceedings of the National Academy of Sciences of the United States of America, 108, 7721-7726

Liu, Q., Gao, X., He, L., \& Lu, W. (2017). Haze removal for a single visible remote sensing image. Signal Processing, 137, 33-43

Long, J., Shi, Z., Tang, W., \& Zhang, C. (2014). Single remote sensing image dehazing. IEEE Geoscience and Remote Sensing Letters, 11, 59-63

Lu, D., Batistella, M., \& Moran, E. (2005). Satellite estimation of aboveground biomass and impacts of forest stand structure. Photogrammetric Engineering \& Remote Sensing, 71, 967-974 Lu, D., Chen, Q., Wang, G., Liu, L., Li, G., \& Moran, E. (2016). A survey of remote sensingbased aboveground biomass estimation methods in forest ecosystems. International Journal of Digital Earth, 9, 63-105

Lumbres, R.I.C., \& Lee, Y.J. (2014). Aboveground biomass mapping of La Trinidad forests in Benguet, Philippines, using Landsat Thematic Mapper data and k-nearest neighbor method. Forest Science and Technology, 10, 104-111 
Mack, B., Leinenkugel, P., Kuenzer, C., \& Dech, S. (2017). A semi-automated approach for the generation of a new land use and land cover product for Germany based on Landsat time-series and Lucas in-situ data. Remote Sensing Letters, 8, 244-253

Magdon, P., \& Kleinn, C. (2013). Uncertainties of forest area estimates caused by the minimum crown cover criterion. Environmental Monitoring and Assessment, 185, 5345-5360

Makarau, A., Richter, R., Muller, R., \& Reinartz, P. (2014a). Haze detection and removal in remotely sensed multispectral imagery. IEEE Transactions On Geoscience and Remote Sensing, 52, 5895-5905

Makarau, A., Richter, R., Müller, R., \& Reinartz, P. (2014b). Haze Detection and Removal in Remotely Sensed Multispectral Imagery. IEEE Transactions On Geoscience and Remote Sensing, 52, 5895-5905

Makarau, A., Richter, R., Schläpfer, D., \& Reinartz, P. (2016). Combined haze and cirrus removal for multispectral imagery. IEEE Geoscience and Remote Sensing Letters, 13, 379-383

Mannschatz, T., Pflug, B., Borg, E., Feger, K.-H., \& Dietrich, P. (2014). Uncertainties of LAI estimation from satellite imaging due to atmospheric correction. Remote Sensing of Environment, 153, 24-39

Marceau, D.J., \& Hay, G.J. (1999). Remote Sensing Contributions to the Scale Issue. Canadian Journal of Remote Sensing, 25, 357-366

McRoberts, R.E., \& Tomppo, E.O. (2007). Remote sensing support for national forest inventories. Remote Sensing of Environment, 110, 412-419

Metz, B., \& Davidson, O.R. (2007). Climate change 2007: Mitigation: Contribution of working group iii to the fourth assessment report of the intergovernmental panel on climate change. Intergovernmental Panel on Climate Change

Moreira, E.P., Valeriano, M.d.M., Sanches, I.D.A., \& Formaggio, A.R. (2016). TOPOGRAPHIC EFFECT ON SPECTRAL VEGETATION INDICES FROM LANDSAT TM DATA: IS TOPOGRAPHIC CORRECTION NECESSARY? Boletim de Ciências Geodésicas, 22, 95-107

Moreira, E.P., \& Valeriano, M.M. (2014). Application and evaluation of topographic correction methods to improve land cover mapping using object-based classification. International Journal of Applied Earth Observation and Geoinformation, 32, 208-217

Morel, A.C., Saatchi, S.S., Malhi, Y., Berry, N.J., Banin, L., Burslem, D., Nilus, R., \& Ong, R.C. (2011). Estimating aboveground biomass in forest and oil palm plantation in Sabah, Malaysian Borneo using ALOS PALSAR data. Forest Ecology and Management, 262, 1786-1798

Pan, Y., Birdsey, R.A., Phillips, O.L., \& Jackson, R.B. (2013). The Structure, Distribution, and Biomass of the World's Forests. Annual Review of Ecology, Evolution, and Systematics, 44, 593622

Pan, Y.D., Birdsey, R.A., Fang, J.Y., Houghton, R., Kauppi, P.E., Kurz, W.A., Phillips, O.L., Shvidenko, A., Lewis, S.L., Canadell, J.G., Ciais, P., Jackson, R.B., Pacala, S.W., McGuire, A.D., Piao, S.L., Rautiainen, A., Sitch, S., \& Hayes, D. (2011). A Large and Persistent Carbon Sink in the World's Forests. Science, 333, 988-993

Panem, C., Baillarin, S., Latry, C., Vadon, H., \& Dejean, P. (2005). Automatic cloud detection on high resolution images. In, Geoscience and Remote Sensing Symposium, 2005. IGARSS'05. Proceedings. 2005 IEEE International (p. 4 pp.): IEEE

Peng, S.S., Piao, S.L., Zeng, Z.Z., Ciais, P., Zhou, L.M., Li, L.Z.X., Myneni, R.B., Yin, Y., \& Zeng, H. (2014). Afforestation in China cools local land surface temperature. Proceedings of the National Academy of Sciences of the United States of America, 111, 2915-2919 
Poli, D., Remondino, F., Angiuli, E., \& Agugiaro, G. (2015). Radiometric and geometric evaluation of GeoEye-1, WorldView-2 and Pléiades-1A stereo images for 3D information extraction. Isprs Journal Of Photogrammetry and Remote Sensing, 100, 35-47

Potapov, P., Yaroshenko, A., Turubanova, S., Dubinin, M., Laestadius, L., Thies, C., Aksenov, D., Egorov, A., Yesipova, Y., \& Glushkov, I. (2008). Mapping the world's intact forest landscapes by remote sensing. Ecology and Society, 13

Qiu, S., He, B., Zhu, Z., Liao, Z., \& Quan, X. (2017). Improving Fmask cloud and cloud shadow detection in mountainous area for Landsats 4-8 images. Remote Sensing of Environment, 199, 107-119

QuangBao, T., \& Hoa, N. (2016). Remote Sensing Application in Forest Monitoring: An Object Based Approach. International Journal of Remote Sensing Applications, 6, 127-135

Reese, H., Nilsson, M., Pahlén, T.G., Hagner, O., Joyce, S., Tingelöf, U., Egberth, M., \& Olsson, H. (2003). Countrywide estimates of forest variables using satellite data and field data from the national forest inventory. AMBIO: A Journal of the Human Environment, 32, 542-548

Riaño, D., Chuvieco, E., Salas, J., \& Aguado, I. (2003). Assessment of different topographic corrections in Landsat-TM data for mapping vegetation types (2003). IEEE Transactions On Geoscience and Remote Sensing, 41, 1056-1061

Richardson, J.J., \& Moskal, L.M. (2011). Strengths and limitations of assessing forest density and spatial configuration with aerial LiDAR. Remote Sensing of Environment, 115, 2640-2651

Richter, R., Kellenberger, T., \& Kaufmann, H. (2009). Comparison of topographic correction methods. Remote Sensing, 1, 184-196

Riihimäki, H., Heiskanen, J., \& Luoto, M. (2017). The effect of topography on arctic-alpine aboveground biomass and NDVI patterns. International Journal of Applied Earth Observation and Geoinformation, 56, 44-53

Romijn, E., Lantican, C.B., Herold, M., Lindquist, E., Ochieng, R., Wijaya, A., Murdiyarso, D., \& Verchot, L. (2015). Assessing change in national forest monitoring capacities of 99 tropical countries. Forest Ecology and Management, 352, 109-123

Saatchi, S., Marlier, M., Chazdon, R.L., Clark, D.B., \& Russell, A.E. (2011). Impact of spatial variability of tropical forest structure on radar estimation of aboveground biomass. Remote Sensing of Environment, 115, 2836-2849

Shilong, P., Guodong, Y., Jianguang, T., Lei, C., Mengtian, H., Yue, L., Ronggao, L., Jiafu, M., B., M.R., Shushi, P., Ben, P., Xiaoying, S., Zhiqiang, X., Ning, Z., ZhenZhong, Z., \& Yingping, W. (2015). Detection and attribution of vegetation greening trend in China over the last 30 years. Global Change Biology, 21, 1601-1609

Smith, W.B. (2002). Forest inventory and analysis: a national inventory and monitoring program. Environmental Pollution, 116, S233-S242

Soenen, S.A., Peddle, D.R., \& Coburn, C.A. (2005). SCS+ C: A modified sun-canopy-sensor topographic correction in forested terrain. IEEE Transactions On Geoscience and Remote Sensing, 43, 2148-2159

Souza Jr, C., Firestone, L., Silva, L.M., \& Roberts, D. (2003). Mapping forest degradation in the Eastern Amazon from SPOT 4 through spectral mixture models. Remote Sensing of Environment, 87, 494-506

Tan, B., Masek, J.G., Wolfe, R., Gao, F., Huang, C., Vermote, E.F., Sexton, J.O., \& Ederer, G. (2013). Improved forest change detection with terrain illumination corrected Landsat images. Remote Sensing of Environment, 136, 469-483 
Tan, K., Piao, S., Peng, C., \& Fang, J. (2007). Satellite-based estimation of biomass carbon stocks for northeast China's forests between 1982 and 1999. Forest Ecology and Management, 240, 114-121

Tang, X. (2015). Estimation of biomass, volume and growth of subtropical forests in Shitai County, China. In: Niedersächsische Staats-und Universitätsbibliothek Göttingen

Tian, X., Su, Z., Chen, E., Li, Z., van der Tol, C., Guo, J., \& He, Q. (2012). Estimation of forest above-ground biomass using multi-parameter remote sensing data over a cold and arid area. International Journal of Applied Earth Observation and Geoinformation, 14, 160-168

Tokola, T., Sarkeala, J., \& Van der Linden, M. (2001). Use of topographic correction in Landsat TM-based forest interpretation in Nepal. International Journal of Remote Sensing, 22, 551-563

Tomppo, E., Olsson, H., Ståhl, G., Nilsson, M., Hagner, O., \& Katila, M. (2008). Combining national forest inventory field plots and remote sensing data for forest databases. Remote Sensing of Environment, 112, 1982-1999

Tropek, R., Sedláček, O., Beck, J., Keil, P., Musilová, Z., Šímová, I., \& Storch, D. (2014). Comment on "High-resolution global maps of 21st-century forest cover change". science, 344, 981-981

Turner, M.G., O'Neill, R.V., Gardner, R.H., \& Milne, B.T. (1989). Effects of changing spatial scale on the analysis of landscape pattern. Landscape Ecology, 3, 153-162

Valeriano, M.d.M., Sanches, I.D.A., \& Formaggio, A.R. (2016). TOPOGRAPHIC EFFECT ON SPECTRAL VEGETATION INDICES FROM LANDSAT TM DATA: IS TOPOGRAPHIC CORRECTION NECESSARY? Boletim de Ciências Geodésicas, 22, 95-107

Vanonckelen, S., Lhermitte, S., \& Van Rompaey, A. (2015). The effect of atmospheric and topographic correction on pixel-based image composites: Improved forest cover detection in mountain environments. International Journal of Applied Earth Observation and Geoinformation, 35, 320-328

Verbesselt, J., Umlauf, N., Hirota, M., Holmgren, M., Van Nes, E.H., Herold, M., Zeileis, A., \& Scheffer, M. (2016). Remotely sensed resilience of tropical forests. Nature climate change, 6 , 1028

Wallner, A., Elatawneh, A., Schneider, T., Kindu, M., Ossig, B., \& Knoke, T. (2018). Remotely sensed data controlled forest inventory concept. European Journal of Remote Sensing, 51, 75-87 Wang, G., \& Gertner, G.Z. (2013). Spatial uncertainty analysis when mapping natural resources using remotely sensed data. Remote Sensing of Natural Resources, 9, 87

Wang, G., Oyana, T., Zhang, M., Adu-Prah, S., Zeng, S., Lin, H., \& Se, J. (2009). Mapping and spatial uncertainty analysis of forest vegetation carbon by combining national forest inventory data and satellite images. Forest Ecology and Management, 258, 1275-1283

Wulder, M. (1998). Optical remote-sensing techniques for the assessment of forest inventory and biophysical parameters. Progress In Physical Geography, 22, 449-476

Wulder, M.A., White, J.C., Nelson, R.F., Næsset, E., Ørka, H.O., Coops, N.C., Hilker, T., Bater, C.W., \& Gobakken, T. (2012). Lidar sampling for large-area forest characterization: A review. Remote Sensing of Environment, 121, 196-209

Ying, Z., \& Guindon, B. (2003). Quantitative assessment of a haze suppression methodology for satellite imagery: effect on land cover classification performance. IEEE Transactions On Geoscience and Remote Sensing, 41, 1082-1089

Zhou, B., Gu, L., Ding, Y., Shao, L., Wu, Z., Yang, X., Li, C., Li, Z., Wang, X., \& Cao, Y. (2011). The great 2008 Chinese ice storm: its socioeconomic-ecological impact and sustainability lessons learned. Bulletin Of The American Meteorological Society, 92, 47-60 
Zhu, Z., Wang, S., \& Woodcock, C.E. (2015). Improvement and expansion of the Fmask algorithm: Cloud, cloud shadow, and snow detection for Landsats 4-7, 8, and Sentinel 2 images. Remote Sensing of Environment, 159, 269-277

Zhu, Z., \& Woodcock, C.E. (2012). Object-based cloud and cloud shadow detection in Landsat imagery. Remote Sensing of Environment, 118, 83-94

Zhu, Z., \& Woodcock, C.E. (2014). Automated cloud, cloud shadow, and snow detection in multitemporal Landsat data: An algorithm designed specifically for monitoring land cover change. Remote Sensing of Environment, 152, 217-234

Zhu, Z., Woodcock, C.E., \& Olofsson, P. (2012). Continuous monitoring of forest disturbance using all available Landsat imagery. Remote Sensing of Environment, 122, 75-91

Zylshal, Sulma, S., Yulianto, F., Nugroho, J.T., \& Sofan, P. (2016). A support vector machine object based image analysis approach on urban green space extraction using Pleiades-1A imagery. Modeling Earth Systems and Environment, 2, 54 
Annex A: Manuscript I 


\section{Moving Window-based Topographic Normalization of Optical Satellite Imagery for Forest Mapping in Mountainous Terrain}

\author{
Dengkui Mo, Hans Fuchs, Lutz Fehrmann, Haijun \\ Yang, Christoph Kleinn \\ Chair of Forest Inventory and Remote Sensing \\ Burckhardt Institute, Georg-August-Universität Göttingen \\ Büsgenweg 5, 37077 Göttingen, Germany \\ dmo@gwdg.de
}

\begin{abstract}
Relief has a significant impact on image classification in mountain areas because slope and aspect of the terrain together with the illumination geometry (solar zenith, solar azimuth angle and sensor position) make that one and the same land cover class has markedly different spectral signatures within one satellite image. Topographic normalization models help reduce intra-class spectral variability. This study proposes and evaluates a moving window-based rotation-correction topographic normalization model. We tested the algorithm with the latest Landsat 8 imagery in a region with very high forest cover in Shitai County, Anhui Province, China, which is characterized by a rough terrain with very steep slopes. Visual comparison and statistical analysis showed that the proposed method yielded better performance at a range of window sizes compared to uncorrected data or global correction methods. The heterogeneity of spectral signatures inside each land cover class could significantly be reduced, which may be partly due to the fact that a site-specific parameterization was used. Model performance was relatively stable over the tested range of window sizes. This new method for parameter estimation for topographic normalization is simple and straightforward, making this technique a suitable option for standard pre-processing of optical satellite imagery.
\end{abstract}

Keywords-Rotation-correction model; empirical parameter estimation; Landsat 8; ASTER GDEM, moving window

\section{INTRODUCTION}

Optical satellite imagery provides large-area environmental monitoring data, but spectral distortion caused by terrain models and solar illumination geometry requires correction. Topographic normalization models have been widely studied for optical satellite imagery to correct the differences in illumination conditions (IC). These models aim to adjust the spectral reflectance values as a function of topographic and illumination characteristics so that image classification is facilitated, and thus more accurate.

Many topographic normalization models have been developed, and most wavelength-dependent models such as C-correction [1], Minnaert [2] and Rotation correction models [3] outperform wavelength-independent ones such as the Cosine model [4]. Recently, some modified models such as modified C-Correction [5] and modified Minnaert [6]

\author{
Yuanchang Lu \\ Research Institute of Forest Resource Information \\ Techniques, Chinese Academy of Forestry, \\ Beijing 100091, PR China \\ ylu@caf.ac.cn
}

have been developed and tested. The performance of such models heavily depends on the quality of empirical parameter estimation (Minnaert constant $k$ for Minnaert models, $c$ factor for C-correction models, and $b$ factor for Rotation-correction models, respectively). These empirical parameters are derived from the relationship between the spectral data and the topographic and illumination characteristics of the terrain.

Previous works have compared these models under different conditions $[1,6,7]$, but their performance is site-dependent, and currently no topographic normalization model is globally accepted. Good performance appear to occur only in relatively small study sites when using one global parameter for all pixels from one band image [1] or when complex pre-stratification approaches were used, under conditions where the relationship between spectral signature and IC can be assumed topography- or illumination-specific [8] or land cover-specific [9]. Sample selection is the key to an unbiased estimation of the empirical model parameters. We found that global parameter estimation methods are prone to over-correction, while land cover-specific methods are too complex for direct application in topographic normalization processing for large-area optical satellite imagery.

In this study, we aimed to develop a simple, generalized, standard topographic normalization model for large-area optical satellite imagery. We hypothesized that the relationship between spectral signature and IC is site-specific and we expected that the empirical parameters of each pixel could be calculated directly from a given window size.

\section{StUdy AREA AND DATASET}

\section{A. Study area}

This study was carried out in the mountainous forested study area of the research project Lin4Carbon in Shitai County, South-Eastern China. The study site has an area of $1412 \mathrm{~km}^{2}\left(29^{\circ} 59^{\prime} 34^{\prime \prime} \mathrm{N}-30^{\circ} 20^{\prime} 31^{\prime \prime} \mathrm{N}\right.$ and $117^{\circ} 12^{\prime} 23^{\prime \prime}$ $\left.\mathrm{E}-117^{\circ} 40^{\prime} 14^{\prime \prime} \mathrm{E}\right)$. Major parts of the study area are characterized by steep slopes and a rugged topography, with elevations ranging from about 4 to $1695 \mathrm{~m}$ (Figure 1). The 


\section{Third International Workshop on Earth Observation and Remote Sensing Applications}

area is covered by a variety of forest types, and due to the complex illumination conditions, similar forest type may exhibit vastly different spectral reflectance characteristics.

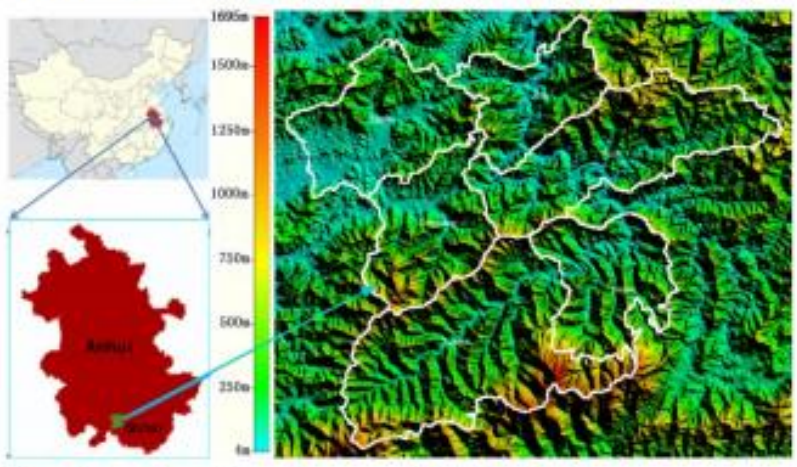

Figure 1. Location of the study area and ASTER GDEM

\section{B. Satellite imagery and DEM}

The Landsat 8 Operational Land Imager (OLI) produces 8 multispectral bands with a spatial resolution of 30 meters. In this study, one cloud free Landsat 8 scene (path/row: 121/39, acquired on 2013-11-22) was selected. In addition, the ASTER Global Digital Elevation Model Version 2 (ASTER GDEM V2) was used, because its spatial resolution is relatively close to that of Landsat 8 optical bands and it has been successfully applied in recent studies [9].

\section{METHODS}

\section{A. Image pre-processing}

The Landsat 8 image and the ASTER GDEM image were pre-processed. Firstly, band data were converted from digital numbers to top of atmosphere (TOA) reflectance using the radiance rescaling factors and solar parameters provided in the metadata file (available at http://landsat.usgs.gov/Landsat8_Using_Product.php). Secondly, the ASTER GDEM model was resampled to $30 \mathrm{~m}$. Finally, slope and aspect data were extracted using topographic modeling with a kernel size of 3 pixels.

\section{B. Illumination condition caculation}

This study focused on one of wavelength-specific or band-specific topographic normalization models-Rotation correction, the method was descripted in Table 1.The first step in this model is to calculate the IC image for each pixel [3], which is calculated from formula (1).

$$
\mathrm{IC}=\cos (Z) \cos (S)+\sin (Z) \sin (S) \cos \left(\varphi_{z}-\varphi_{S}\right)
$$

where $Z$ is the solar zenith angle and $\varphi_{z}$ is the solar azimuth angle. These two solar parameters are constant over the entire image and can directly be read from the metadata file of Landsat 8. $S$ is the topographic slope angle and $\varphi_{S}$ is the aspect angle of the topographic surface, and they are produced from the ASTER GDEM. The value of IC ranges from -1 to 1 . IC $=\cos (Z)$ for a horizontal surface.

Table 1. The general rotation-correction model

\begin{tabular}{l|l|l|l|l}
\hline Type & Formula & Reference & Linear regression & Parameters \\
\hline Rotation-correction & $L_{H}(\lambda)=L_{I}(\lambda)-a(\lambda) *(\mathrm{IC}-\cos (Z))$ & {$[3]$} & $L_{I}(\lambda)=a(\lambda) * \mathrm{IC}+b(\lambda)$ & $a(\lambda)$ \\
\hline
\end{tabular}

$L_{H}=$ Corrected reflectance (for a horizontal surface); $L_{I}=$ Observod reflectance on the incline surface; $\lambda=$ Specific wavelength band; $a(\lambda)=$ Slope of the linear regression for $\lambda ; b(\lambda)=$ Intercept of the linear regression for $\lambda$.

\section{Moving window-based topographic normalization}

Empirical parameter estimation is the critical step in the model mentioned in Table 1. Here, we demonstrate a moving window-based method derived from the Rotation-correction model. It is a pixel-based estimation method using a moving window dataset.

For each pixel, the parameter $a(\lambda)_{i, j}$ is estimated based on linear regression using dataset IC and $L_{I}(\lambda)$, located in $[i-k, i+k],[j-k, j+k]$

$$
\begin{aligned}
& L_{I}(\lambda)_{[i-k, i+k],[j-k, j+k]} \\
& =a(\lambda)_{i, j} * \mathrm{IC}_{[i-k, i+k],[j-k, j+k]}+b(\lambda)_{i, j}
\end{aligned}
$$

where cell $i, j$ (row $i$ and column $j$ ) is the center pixel of the moving window, and $k=$ kernel size for a window size of $(2 * k+1)^{2}$.
Here, $p \%$ of the pixels in window $i, j$ are reused in the next window of $i+1, j$ or $i, j+1$ as shown in formula (3). For example, when $k=25, p \% \approx 98 \%$.

$$
p=\frac{(2 * k+1)^{2}-(2 * k+1)}{(2 * k+1)^{2}} * 100
$$

The Pearson correlation coefficient $r_{i, j}$ was calculated for each moving window center pixel. In order to evaluate the quality of $a(\lambda)$ from separated bands, $r$ was computed for each band.

Finally, referring to Table 1:

$$
L_{H}(\lambda)=L_{I}(\lambda)-a(\lambda) *(\mathrm{IC}-\cos (Z))
$$

was used to produce the corrected imagery. 


\section{Third International Workshop on Earth Observation and Remote Sensing Applications}

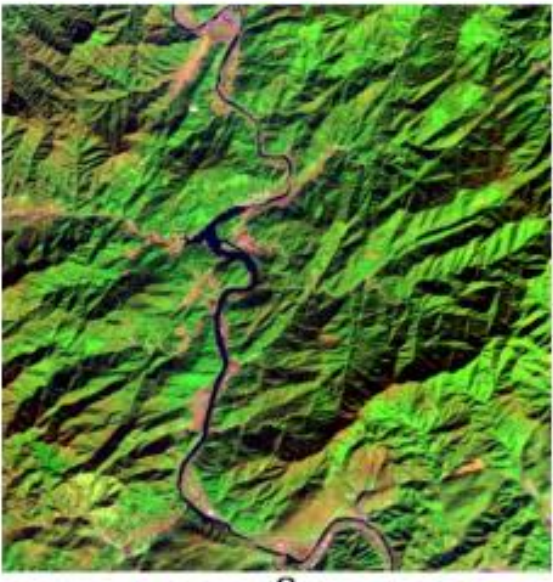

a

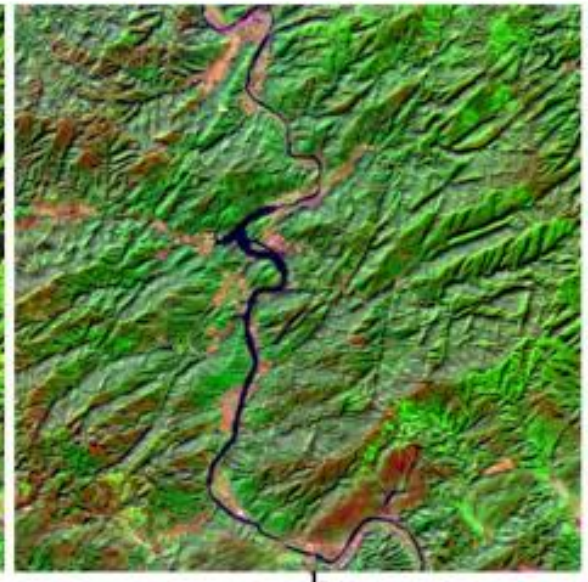

b

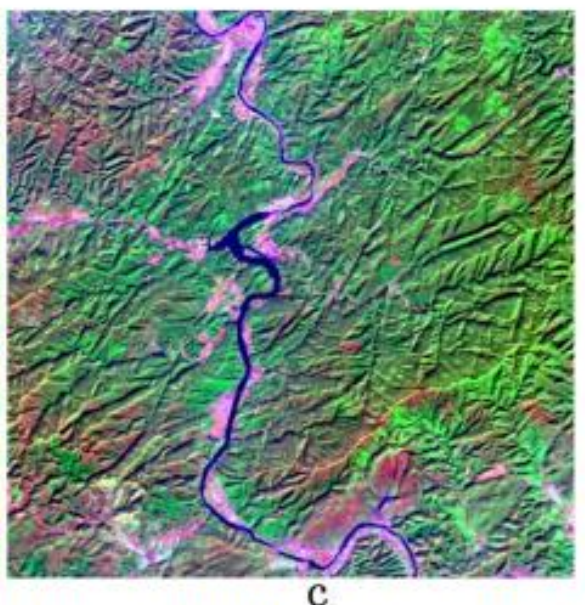

C

Figure 2. Visual assessment (RGB = 7, 5, 3)for (a) Original Landsat 8, (b) Rotation-correction based on a global parameter, and (c) Moving window-based method with kernel size $=50$.

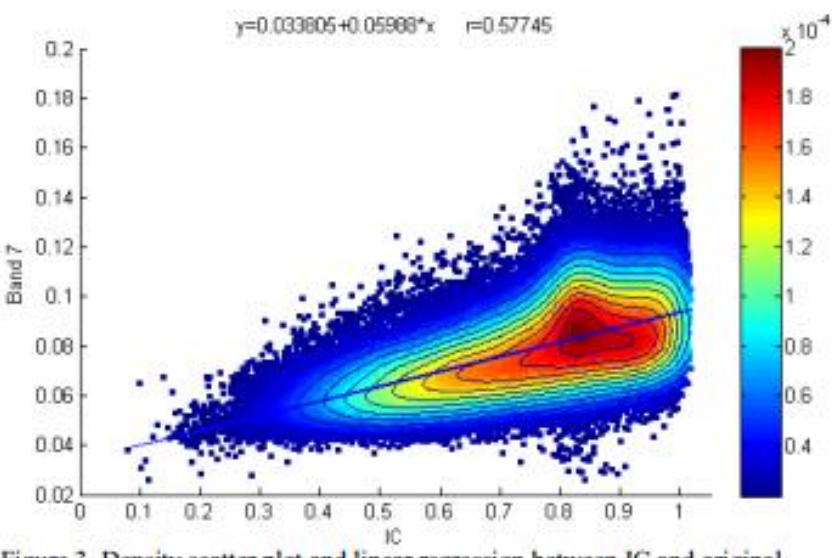

Figure 3. Density scatter plot and linear regression between IC and original Band 7. A global parameter $a($ band 7$)=0.05988$ was used in formula (5) to produce the corrected image in Figure 2b, with $r=0.57745$.

\section{RESULTS}

\section{A. Visual Assessment}

Visual comparison is one of most direct assessment methods. Figure 2 shows that the effect of the terrain on the spectral signature has been reduced when the moving window-based method was used. Compared to the global parameter estimation method we found that no obvious over-correction did occur. The boundaries of land cover types were preserved, even in rough terrain. This may be because $p \%$ of the pixels were reused in parameter estimation of adjacent pixels, which can have a smoothing effect.

\section{B. Statistical Analysis}

Theoretically, the correlation between IC and pixel spectral values for each band may be low if similar IC regions are covered with different land types or if one land cover type is distributed over a wide range of IC. Figure 3 shows the density scatter plot and linear regression between IC and Band 7 from the study area, and the global
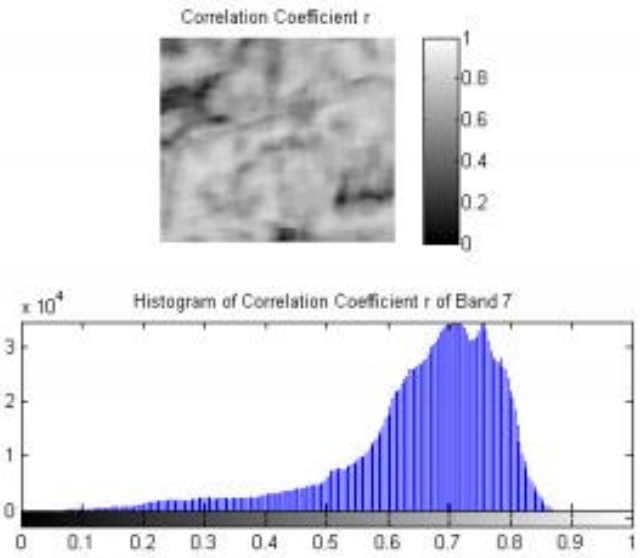

Figure 4. Pearson correlation coefficient $r$ spatial image and histogram of Band 7. Here, $r$ is estimated based on a moving window with $k=50$.

parameter $a($ band 7$)=0.05988$ was used to produce an over-corrected image (Figure 2b). In contrast, the moving window based method firstly produced the $a$ (band 7 ) image using formula (2), and then calculated the corrected image Figure 2c using formula (4). Furthermore, the Pearson correlation coefficient $r$ and the histogram of band 7 are shown in Figure 4 demonstrated that $81.38 \%$ pixels in band 7 had $r>0.57745$, and that Figure 2c indicates better results than Figure $2 b$. Our interpretation of these results is that the quality of topographic normalization is positively correlated with $r$ Therefore, the image map of $r$ and its histogram could be applied to evaluating the quality of the topographic normalization.

We analyzed intra-class variability using pairs of box plots. Box plots illustrate the spread and differences between samples using the 25th, 50th and 75th percentiles-also known as the lower quartile (Q1), median (Q2) and upper quartile $(\mathrm{Q} 3)$ - and the interquartile range $(\mathrm{IQR}=\mathrm{Q} 3-\mathrm{Q1})$, which covers the central $50 \%$ of the data [10]. Figure 5 exhibits the TOA distribution differences between original 


\section{Third International Workshop on Earth Observation and Remote Sensing Applications}

imagery and corrected imagery for six land cover types. In general, IQRs for the corrected imagery were narrower and located within the range of IQRs for the original imagery. The exception was the land cover type "buildings, which had IQRs of similar sizes for corrected and original imagery. This may be because the buildings were located in flat areas with similar illumination condition. These findings indicate that imagery for the majority of land covers becomes more homogeneous in corrected imagery based on the moving window method.

Intra-class heterogeneity or variability was expressed as the coefficient of variation. We expected that a good topographic normalization model could help reducing the spectral variability of each land cover type. Therefore, CV\% could be an index for evaluating the quality of topographic normalization models due to the fact that high $\mathrm{CV} \%$ means high heterogeneity. In this study, CV\% of four main forest types was calculated for different methods across different bands. Figure 6 shows that the $\mathrm{CV} \% \mathrm{~s}$ of the moving window based methods are much lower than those of the rotation correction model and of the original TOA images across all bands and forest types. This suggests that the performance of the proposed method is better than the others in this study. And $\mathrm{CV} \%$ of rotation correction model is obviously higher than those of original TOA images from band 1 to band 4 . This means that the general rotation correction model cannot yield good results from all the bands.

In addition, the influence of window size on topographic normalization was assessed by testing a range of window sizes. We assumed that no strong kernel size sensitivity exists between images produced using different kernel sizes if their $\mathrm{CV} \% \mathrm{~s}$ are stable. $\mathrm{CV} \%$ of moving window based methods with 3 kernel sizes were almost the same in all the bands and forest types in Figure 6.

\section{Discussion AND CONCLUSIONS}

\section{A. Novel topographic normalization model}

Based on the performance analysis between a traditional rotation-correction model and the new moving window-based topographic normalization algorithm, the proposed model is a simple, more robust, and more effective topographic normalization model.

The proposed method is a band-specific topographic normalization model and it was based on the hypothesis that the relationship between spectral features and illumination conditions is site-specific. Our results support this hypothesis and indicate that this approach is more accurate than those using global parameter estimation methods. More importantly, the proposed method does not require a priori parameter information; this indicates the generalizability of the algorithm.

\section{B. Opportunities for Development}

Some challenges remain unresolved. Firstly, although the demonstrated robustness of window size selection makes image quality controllable, optimization and evaluation of window size selection are still needed. Secondly, the algorithm's computational demand is very high, as the model executes all steps of global parameter estimation method pixel by pixel. Thus, the computing time increases linearly with the number of pixels in an image. Parallel computing design and further development could help solve this problem. Thirdly, to fully demonstrate generalizability, this site-specific approach needs further application to other models, such as the Minnaert or C-correction models. Finally, the performance of the proposed method for applications in vegetation classification in different study areas requires further testing.

\section{ACKNOWLEDGMENT}

This research was jointly supported by the German Federal Ministry of Education and Research (BMBF), (Project Lin4Carbon Grant No. 033L049C), the Chinese Academy of Forestry (CAF) (Grant No. Lin2Value -CAFYBB2012013) and the National Natural Science Foundation of China (Grant No. 31100412). We thank PhD student Xiaolu Tang from Georg-August-Universität Göttingen, for field work. We also thank the Master students Ms. Ping Wu and Ms. Ningxin Lu from Anhui Agriculture University, for field data collection in the very steep mountain forests of Shitai County. And finally our great thanks go to the Shitai Forest Bureau for a very efficient cooperation and a perfect logistical support of our research.

\section{REFERENCES}

[1] Riano, D., Chuvieco, E., Salas, J., and Aguado, I.: 'Assessment of different topographic corrections in Landsat-TM data for mapping vegetation types (2003)', Ieee Transactions on Geoscience and Remote Sensing, 2003, 41, (5), pp. 1056-1061

[2] Lu, D.S., Ge, H.L., He, S.Z., Xu, A.J., Zhou, G.M., and Du, H.Q.: 'Pixel-based Minnaert Correction Method for Reducing Topographic Effects on a Landsat 7 ETM+ Image', Photogrammetric Engineering and Remote Sensing, 2008, 74, (11), pp. 1343-1350

[3] Tan, B., Masek, J.G., Wolfe, R., Gao, F., Huang, C.Q., Vermote, E.F., Sexton, J.O., and Ederer, G.: 'Improved forest change detection with terrain illumination corrected Landsat images', Remote Sensing of Environment, 2013, 136, pp. 469-483

[4] Civoo, D.L.: 'Topographic Normalization of Landsat Thematic Mapper Digital Imagery', Photogrammetric Engineering and Remote Sensing, 1989, 55, (9), pp. 1303-1309

[5] Hantson, S., and Chuvieco, E.: 'Evaluation of different topographic correction methods for Landsat imagery', International Journal of Applied Earth Observation and Geoinformation, 2011, 13, (5), pp. 691-700

[6] Richter, R., Kellenberger, T., and Kaufmann, H.: 'Comparison of Topographic Correction Methods', Remote Sensing, 2009, 1, (3), pp. $184-196$

[7 Gao, M.-L., Zhao, W.-J., Gong, Z-N., Gong, H.-L., Chen, Z., and Tang, X.-M.: 'Topographic Correction of ZY-3 Satellite Images and Its Effects on Estimation of Shrub Leaf Biomass in Mountainous Areas', Remote Sensing, 2014, 6, (4), pp. 2745-2764

[8] Reese, H., and Olsson, H.: 'C-correction of optical satellite data over alpine vegetation areas: A comparison of sampling strategies for determining the empirical c-parameter', Remote Sensing of Environment, 2011, 115, (6), pp. 1387-1400

[9] Szantoi, Z., and Simonetti, D.: 'Fast and Robust Topographic Correction Method for Medium Resolution Satellite Imagery Using a Stratified Approach', leee Journal of Selected Topics in Applied Earth Observations and Remote Sensing, 2013, 6, (4), pp. 1921-1933

[10] Krzywinski, M., and Altman, N.: 'Visualizing samples with box plots', Nat Methods, 2014, 11, (2), pp. 119-120 
2014 Third International Workshop on Earth Observation and Remote Sensing Applications
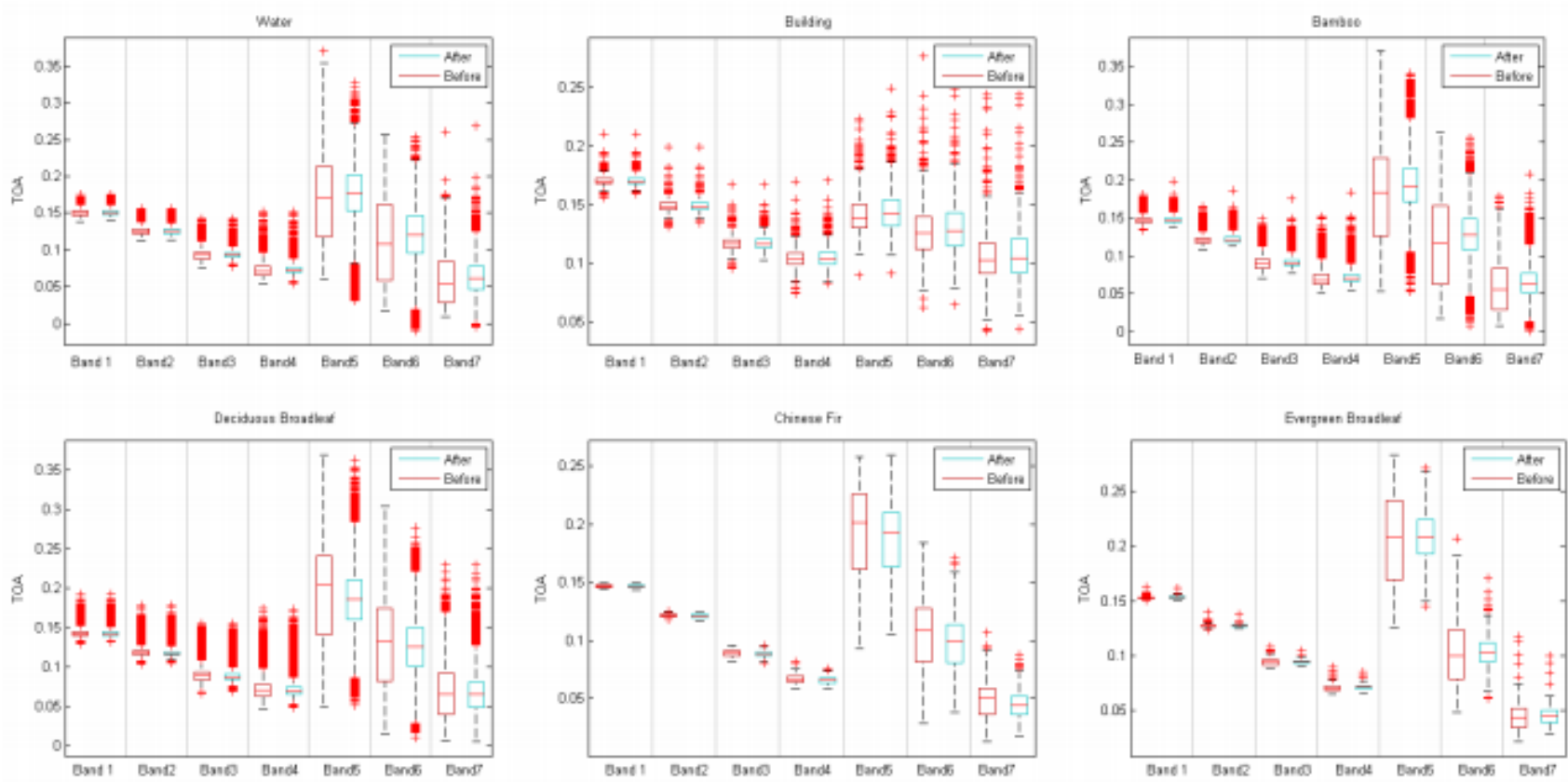

Figure 5. Box-plot comparisons of TOA for original and corrected imagery ( $k=50$, using moving window method) across different bands and land cover types. Ground truth samples were collected from the field. Sample size of each land cover type ranges from 223 to 1054 pixels.
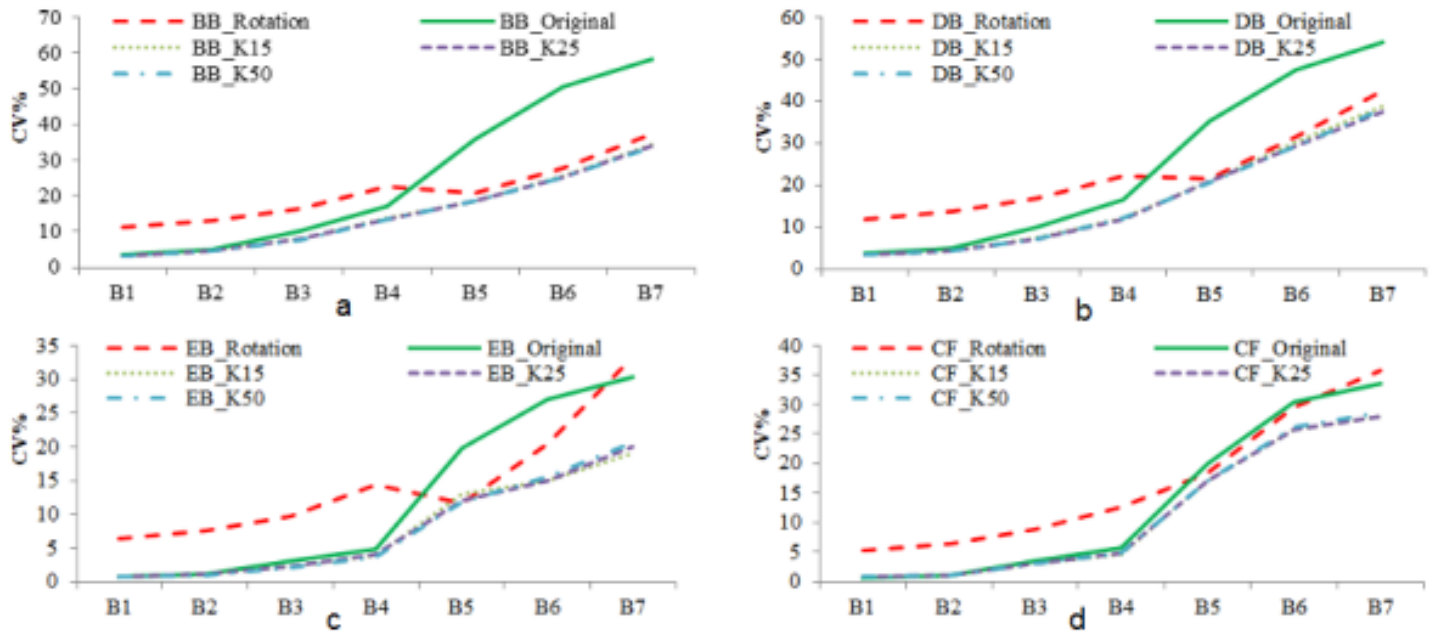

Figure 6. Coefficient of variation (CV\%) for samples of 4 main forest types from original, rotation-correction and moving window based on different methods across different bands. BB, DB, EB and CF: samples from bamboo, deciduous broadleaf, evergreen broadleaf and Chinese fir. Rotation, original, K15, K25, K50: samples of images based on rotation correction model, original TOA images and moving window based methods with kernel size $=15,25,50$ pixels respectively. Ground truth samples were collected from the field. Sample size of each land cover type ranges from 223 to 1054 pixels. $\mathrm{CV} \%=(\mathrm{SD} / \mathrm{mean} * 100 \%)$. 
Annex B: Manuscript II 


\title{
Local Parameter Estimation of Topographic Normalization for Forest Type Classification
}

\author{
Dengkui Mo, Hans Fuchs, Lutz Fehrmann, Haijun Yang, Yuanchang Lu, and Christoph Kleinn
}

\begin{abstract}
Radiometric distortions caused by rugged terrain make the classification of forest types from satellite imagery a challenge. Various band-specific topographic normalization models are expected to eliminate or reduce these effects. The quality of these models also depends on the approach to estimate empirical parameters. Generally, a global estimation of these parameters from a whole satellite image is simple, but it may tend to overcorrection, particularly for larger areas. A land-cover-specific method usually performs better, but it requires obtaining a priori land classification, which presents another challenge in many cases. Empirical parameters can be directly estimated from local pixels in a given window. In this letter, we propose and evaluate a central-pixel-based parameter estimation method for topographic normalization using local window pixels. We tested the method with Landsat 8 imagery and the Advanced Spaceborne Thermal Emission and Reflection Radiometer Global Digital Elevation Model (ASTER GDEM) in very rough terrain with diverse forest types. Visual comparison and statistical analyses showed that the proposed method performed better at a range of window sizes compared with an uncorrected image or with a global parameter estimation approach. The intraclass spectral variability of each forest type has been reduced significantly, and it can yield higher accuracy of forest type classification. The proposed method does not require the a priori knowledge of land covers. Its simplicity and robustness suggest that this method has the potential to be a standard preprocessing approach for optical satellite imagery, particularly for rough terrain.
\end{abstract}

Index Terms-Advanced Spaceborne Thermal Emission and Reflection Radiometer Global Digital Elevation Model (ASTER GDEM), empirical parameter estimation, Landsat 8, local window, topographic normalization.

\section{INTRODUCTION}

O PTICAL satellite imagery provides large-area environmental monitoring data, but a spectral distortion caused by terrain models and solar illumination geometry requires correction. Topographic normalization models have been widely studied for optical satellite imagery to correct the differences

Manuscript received January 30, 2015; revised May 27, 2015; accepted June 20, 2015. Date of publication July 14, 2015; date of current version August 7, 2015. This work was supported in part by the German Federal Ministry of Education and Research (BMBF) through the Lin4Carbon Project under Grant $033 \mathrm{~L} 049 \mathrm{C}$, by the Chinese Academy of Forestry under Grant Lin2Value-CAFYBB2012013, and by the National Natural Science Foundation of China under Grant 31470643 and Grant 31100412.

D. Mo, H. Fuchs, L. Fehrmann, H. Yang, and C. Kleinn are with the Chair of Forest Inventory and Remote Sensing, Burckhardt Institute, Faculty of Forest Sciences and Forest Ecology, University of Göttingen, 37077 Göttingen, Germany (e-mail: dmo@gwdg.de; ckleinn $\Theta$ gwdg.de).

Y. $\mathrm{Lu}$ is with the Research Institute of Forest Resource Information Techniques, Chinese Academy of Forestry, Beijing 100091, China (e-mail: ylu@caf.ac.cn).

Color versions of one or more of the figures in this paper are available online at http://ieeexplore.iece.org.

Digital Object Identifier 10.1109/LGRS.2015.2448937 in illumination conditions (ICs). These models aim to adjust the spectral reflectance values as a function of topographic and illumination characteristics so that image classification is facilitated and thus more accurate.

Many topographic normalization models have been developed, and most wavelength-dependent models such as C-correction [1], Minnaert [2], and rotation correction models [3] outperform wavelength-independent models such as the cosine model [4]. Recently, some modified models such as modified C-correction [5] and modified Minnaert [6] models have been developed and tested. The performance of such models heavily depends on the quality of empirical parameter estimation (Minnaert constant $k$ for Minnaert models, $c$ factor for C-correction models, and $b$ factor for rotation correction models). These empirical parameters are derived from the relationship between the spectral data and the topographic and illumination characteristics of the terrain.

Previous studies have compared these models under different conditions [1], [6], [7], but their performance is site dependent, and currently, no topographic normalization model is generally accepted. Good performance appears to only occur in relatively small study sites when using one global parameter for all pixels from one band image [1] or when complex prestratification approaches were used under conditions where the relationship between the spectral signature and an IC can be assumed topography specific or illumination specific [8], or land cover specific [9]. Sample selection is the key to an unbiased estimation of empirical model parameters. We found that global parameter estimation methods are prone to overcorrection, whereas landcover-specific methods are too complex to be directly applied in the topographic normalization of large-area optical satellite imagery.

In this letter, we aimed to develop a simple generalized standard topographic normalization model for large-area optical satellite imagery. We assumed that the relationship between the spectral signature and an IC is site specific, and the empirical parameters for each pixel could be directly calculated from a given window size.

\section{Study Area And Data Set}

\section{A. Study Area}

The study was carried out in the mountainous forest in Shitai County, Southeastern China. The study site has an area of $1412 \mathrm{~km}^{2}\left(29^{\circ} 59^{\prime} 34^{\prime \prime} \mathrm{N}-30^{\circ} 20^{\prime} 31^{\prime \prime} \mathrm{N}\right.$ and $117^{\circ} 12^{\prime} 23^{\prime \prime}$ $\left.\mathrm{E}-117^{\circ} 40^{\prime} 14^{\prime \prime} \mathrm{E}\right)$. Major parts of the study area are characterized by steep slopes and a rugged topography, with elevations ranging from about 0 to $1695 \mathrm{~m}$ (see Fig. 1). The area is covered by a variety of forest types, and due to complex ICs, similar forest 


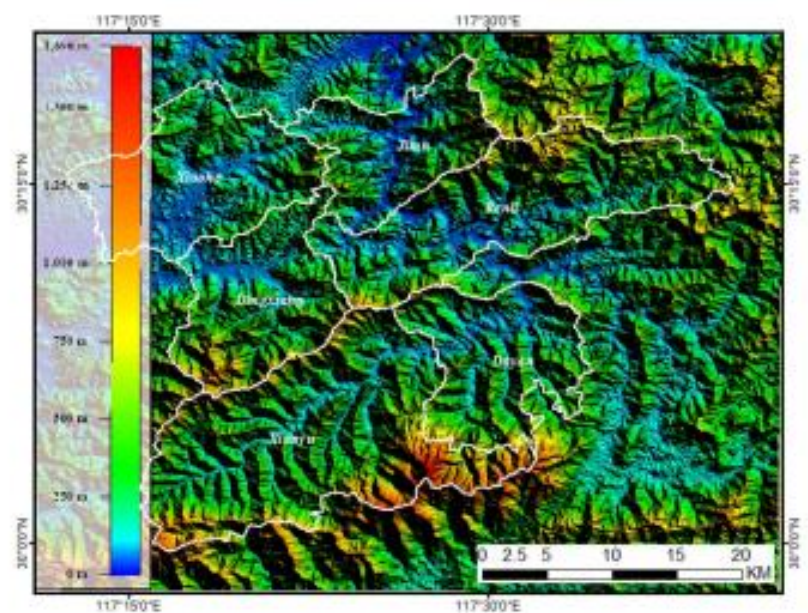

Fig. 1. Illustration of the terrain conditions of the study area produced from the ASTER GDEM.

types may exhibit vastly different characteristics of spectral reflectance.

\section{B. Satellite Imagery and DEM}

The Landsat 8 Operational Land Imager produces 8 multispectral bands with a spatial resolution of $30 \mathrm{~m}$. In this letter, one cloud-free Landsat 8 scene (path/row: 121/39, acquired on March 30,2014) was selected. In addition, the Advanced Spaceborne Thermal Emission and Reflection Radiometer Global Digital Elevation Model (ASTER GDEM) Version 2 (ASTER GDEM V2) was used because its spatial resolution is relatively close to that of Landsat 8 optical bands and because it has been successfully applied in recent studies [9].

\section{METHODS}

\section{A. Image Preprocessing}

The Landsat 8 image and the ASTER GDEM image were preprocessed. First, band data were converted from digital numbers to top of atmosphere (TOA) reflectance using the radiance rescaling factors and solar parameters provided in the metadata file (for conversion to TOA methods, see http://landsat. usgs.gov/Landsat8_Using_Product.php). Second, the ASTER GDEM was resampled to $30 \mathrm{~m}$ using a nearest neighbor sampling method. Finally, slope and aspect images were produced using topographic modeling with a kernel size of 5 pixels $x$ 5 pixels in practice.

\section{B. IC Calculation}

This letter focuses on one of the wavelength-specific or band-specific topographic normalization models, i.e., rotation correction, and the method was described in Table I. The first step in this model is to calculate the IC image for each pixel [3], which is calculated from the following formula:

$$
\mathrm{IC}=\cos (Z) \cos (S)+\sin (Z) \sin (S) \cos \left(\varphi_{z}-\varphi_{s}\right)
$$

where $Z$ is the solar zenith angle, and $\varphi_{z}$ is the solar azimuth angle. These two solar parameters are approximately constant
TABLE I

GENERAL ROTATION CORRECTION MODEL. MAINLY REFER TO THE WORK IN [3]

\begin{tabular}{l|l}
\hline Formula & $L_{H}(\lambda)=L_{I}(\lambda)-a(\lambda)+(\mathrm{IC}-\cos (z))$ \\
\hline Linear regression & $L_{I}(\lambda)=a(\lambda) * \mathrm{IC}+b(\lambda)$ \\
\hline Parameter & $a(\lambda)$ \\
\hline
\end{tabular}

Comelation Coefficient $r$ image

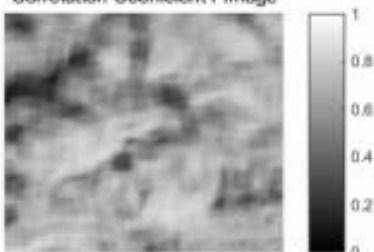

Histogram of Correlation Coefficient $r$ of Band 5

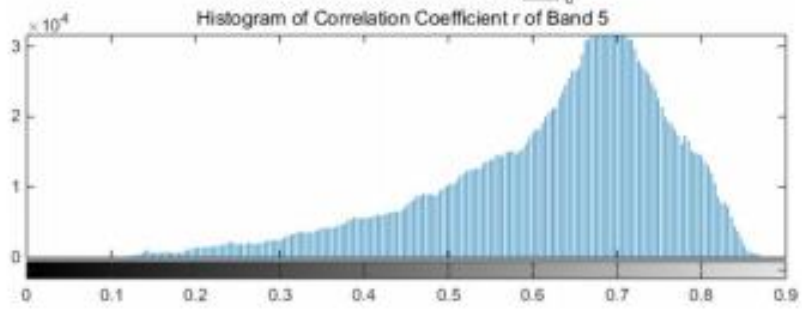

Fig. 2. Pearson correlation coefficient $r(\lambda)$ spatial image and histogram of Band 5. Here, the $r(\lambda)$ image is estimated based on a moving window with $k=50$.

over the entire image and can be directly read from the metadata file of Landsat 8. $S$ is the topographic slope angle, and $\varphi_{s}$ is the aspect angle of the topographic surface; they are produced from the ASTER GDEM. The value of the IC ranges from -1 to 1 . $\mathrm{IC}=\cos (Z)$ for a horizontal surface.

\section{Local Estimation of Parameters}

Empirical parameter estimation is the critical step in the rotation correction model. Here, we modify it to a movingwindow-based method. It is pixel based and uses local window data sets.

For each pixel, parameter $a(\lambda)_{i, j}$ is estimated based on linear regression using data set IC and $L_{I}(\lambda)$, which are located in $[i-k, i+k],[j-k, j+k]$ as follows:

$$
\begin{array}{r}
L_{I}(\lambda)_{[i-k, i+k],[j-k, j+k]} \\
=a(\lambda)_{i, j}{ }^{*} \operatorname{IC}_{[i-k, i+k],[j-k, j+k]}+b(\lambda)_{i, j}
\end{array}
$$

where cell $(i, j)$ (row $i$ and column $j$ ) is the center pixel of the moving window, and $k$ is the kernel size for a window size of $\left(2^{*} k+1\right)^{2}$. At the end, $a(\lambda)$ was produced for each pixel of the study area, and this is in contrast to other techniques (we named it the "global parameter estimation method") that use a fixed parameter for the entire study area.

Here, $p \%$ of the pixels in window $(i, j)$ are reused in the next window of $(i+1, j)$ or $(i, j+1)$, as shown in (3). For example, when $k=25, p \% \approx 98 \%$ as follows:

$$
p=\frac{\left(2^{*} k+1\right)^{2}-\left(2^{*} k+1\right)}{\left(2^{*} k+1\right)^{2}} * 100 .
$$




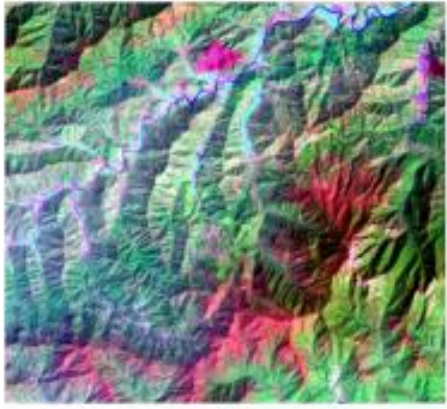

(a)

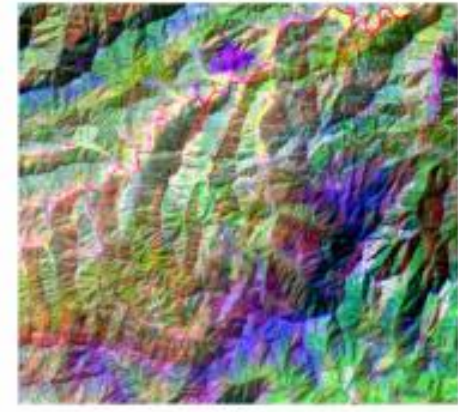

(b)

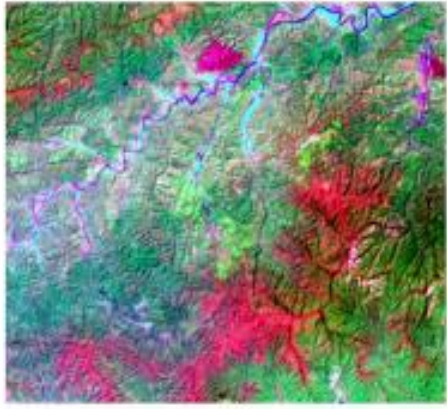

(c)

Fig. 3. Visual comparison (Band composition = Bands 7,5 , and 3 using $2 \%$ linear stretch) for (a) original Landsat 8 , (b) rotation correction based on a global parameter, and (c) moving-window-based method with the kernel size $=50$ pixels. Note that (b) was produced from the manually selected cloud-free part of the Landsat 8 scene.
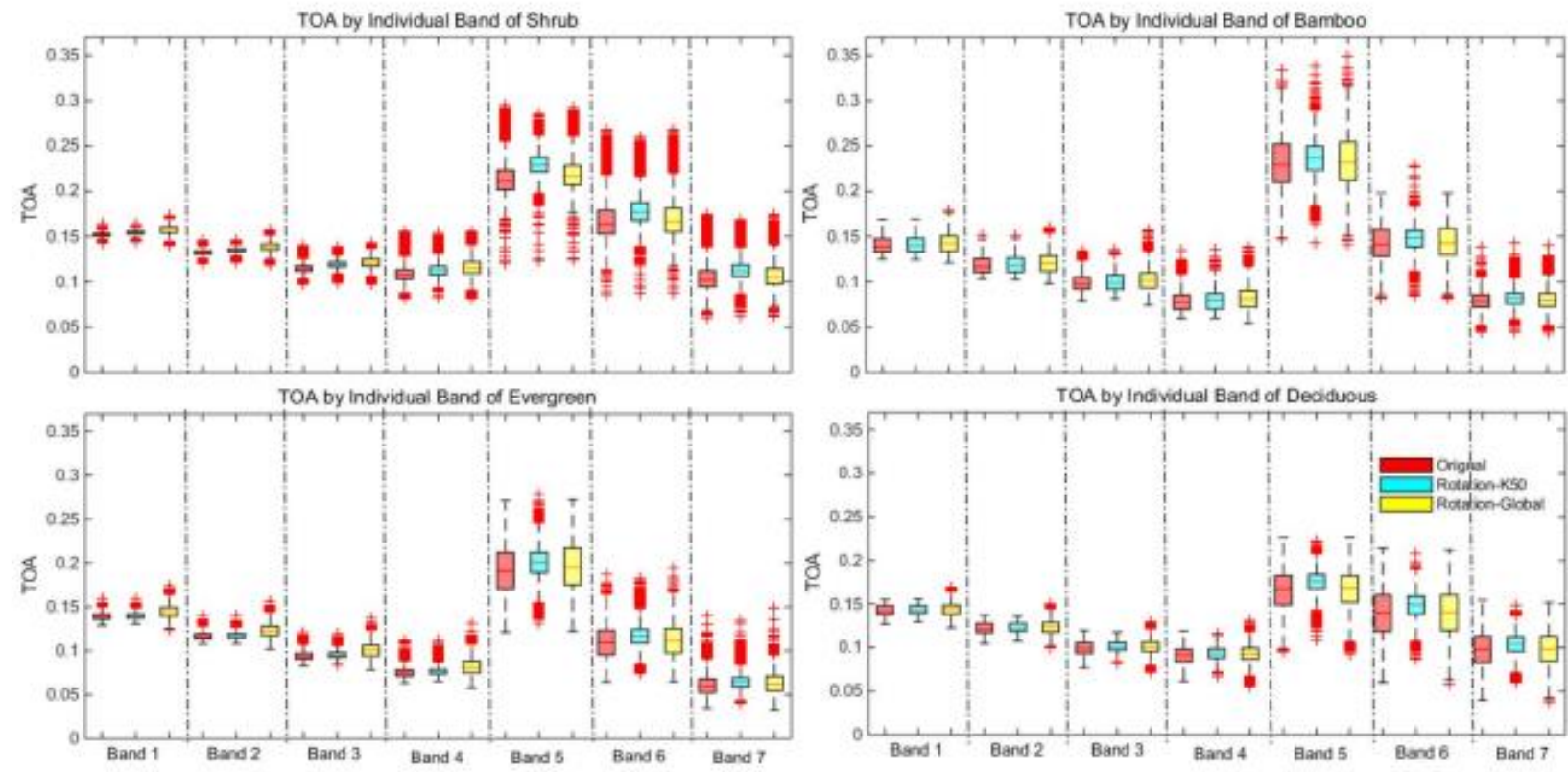

Fig. 4. Box-plot comparisons of the TOA for the original and corrected images from the rotation global and proposed methods $(k=50$ using the moving window method) across different bands and forest types. The ground-truth samples were collected from the field. The sample size of each land-cover type ranges from 3475 to 11945 pixels.

Theoretically, the correlation between the IC and pixel spectral values for each band may be low if similar IC regions are covered with different land covers or if one land-cover type is distributed over a wide range of ICs. Here, Pearson correlation coefficient $r_{i, j}$ was calculated for each center pixel of the moving window. In order to evaluate the quality of $a(\lambda)$ for different bands, an $r(\lambda)$ image was produced for each band. Similar to $a(\lambda), r(\lambda)$ was produced for each pixel of the study area.

Image $r(\lambda)$ and the histogram of image $r(\lambda)$ for Band 5 are shown in Fig. 2, demonstrating that $79.11 \%$ of the pixels in Band 5 had $r>0.5241$. Furthermore, Fig. 3(c) indicates better results than Fig. 3(b). Our interpretation of these results is that the quality of the topographic normalization is positively correlated with $r(\lambda)$. Therefore, the image of $r(\lambda)$ and its histogram could be applied to evaluate the quality of the topographic normalization.

\section{Pixelwise Topographic Normalization}

Finally, referring to Table I, the following was used to produce the corrected image [see Fig. 3(c)]:

$$
L_{H}(\lambda)=L_{I}(\lambda)-a(\lambda) *(\mathrm{IC}-\cos (Z)) .
$$

\section{RESULtS}

\section{A. Visual Assessment}

Visual comparison is one of the most direct assessment methods. Fig. 3 shows that the effect of the terrain on the spectral signature has been reduced with the moving-windowbased method. Compared with the global parameter estimation method, we found that no obvious overcorrection occurred and that the spectral characters of forest types were more consistent with the original image in the illuminated region. 

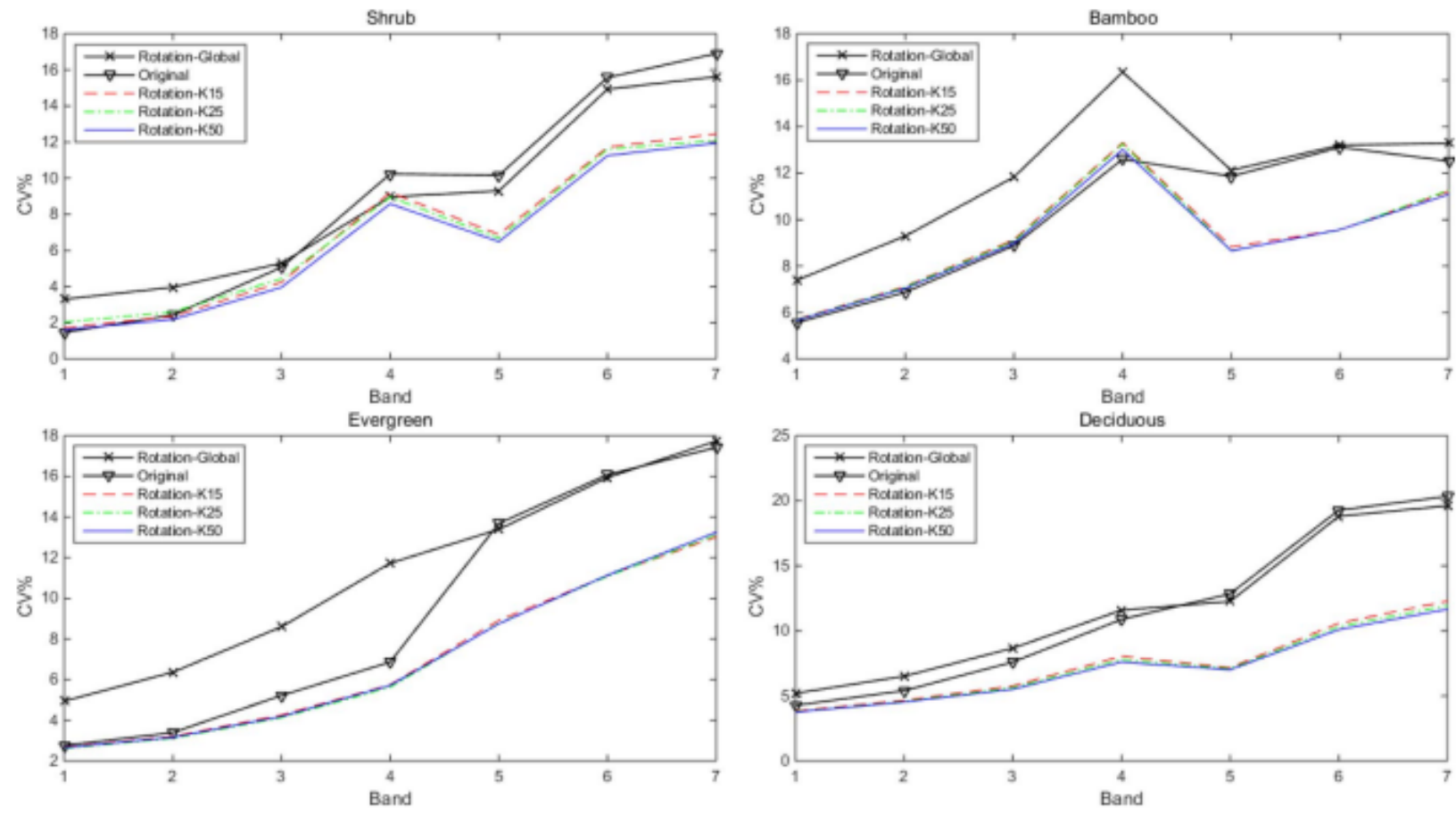

Fig. 5. CV\% for the samples of the four main forest types from images using different methods across Bands 1-7. The ground-truth samples were collected from the field. The sample size of each land-cover type ranges from 3475 to 11945 pixels. CV $=$ Standard Deviation/Mean.

The boundaries of the land-cover types were preserved, even in rough terrain. This may be due to the fact that $p \%$ pixels were reused in the parameter estimation of adjacent pixels, which can have a smoothing effect.

\section{B. Statistical Analysis}

We analyzed the intraclass variability using pairs of box plots. Box plots illustrate the spread and differences between samples using the 25th, 50th, and 75th percentiles, i.e., the interquartile range (IQR), which covers the central $50 \%$ of the data [10]. Fig. 4 exhibits the TOA distribution differences between the original imagery and the two corrected images for four forest types. In general, the IQRs of the four forest types from the rotation-K50 image were narrower than those from most of the bands (particularly for the vegetation-related bands: Band 5-near infrared and Band 4-red) of the original image and the rotation global image. These characteristics are not very obvious from Band 1-ultrablue. However, most of the IQRs from the rotation global image are much larger than those from the original image. These findings indicate that the imagery for majority of the forest types becomes more homogeneous after correction with the moving window method.

The intraclass variability was expressed based on the coefficient of variation (CV). We expected that a good topographic normalization model could help in reducing the spectral variability of each land-cover type. Therefore, CV\% could be an index for evaluating the quality of topographic normalization models due to the fact that a high $\mathrm{CV} \%$ means high variability. In this letter, the CV\% of the four main forest types was calculated for different methods across different bands. Fig. 5 shows

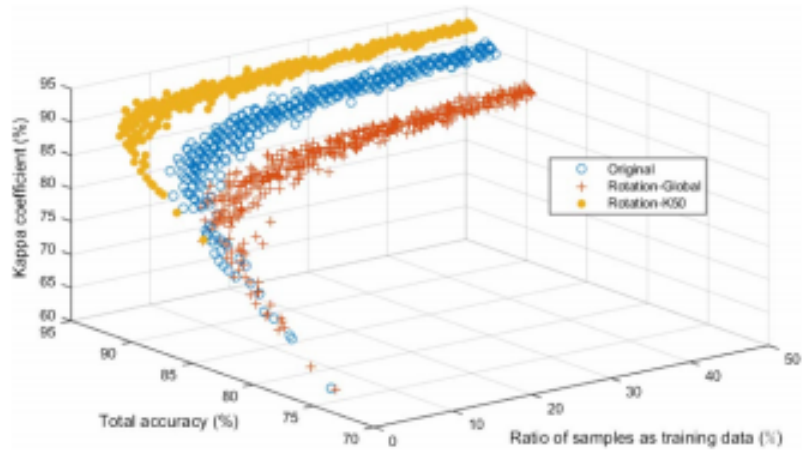

Fig. 6. Performance comparison of the forest type classification simulation (band compositions $7,5,4,3$, and 2 , and $k=5$ with the $k \mathrm{NN}$ classifier).

that the CV\% of the moving-window-based methods is much lower than those of the rotation global model and the original TOA images across Bands 1-7 and four forest types. This suggests that the performance of the proposed method is better compared with the others in this letter. The CV\% of the rotation global model is obviously higher than those of the original TOA images from Bands 1-4. This means that the global parameter estimation method cannot yield good results for all the bands.

In addition, the CV\% values of the moving-window-based methods with three kernel sizes were almost the same in all the bands and forest types. Their CV\% values are stable, i.e., no strong kernel size sensitivity exists between the images produced using different kernel sizes (see Fig. 5). 


\section{Classification Simulation of Forest Types}

We are interested in the performance in forest type classification, and we expected that the decrease in the intraclass spectral variability would help in improving the accuracy. In order to compare the performance of the proposed method with that of the global parameter estimation topographic normalization independently, a large number of forest type pixels (7698 pixels of bamboo, 11254 pixels of deciduous, 11945 pixels of evergreen, and 3475 pixels of shrubs) were selected for training and testing samples. These pixels were collected from four pure forest types in both illuminated terrain and shaded rough terrain, and their radiometric distortion was analyzed in the previous section. A $k$-nearest neighbor $(k N N)$ classifier was used for classification simulation as it is a nonparametric classifier without any assumption on statistical parameters, which labels an unclassified pixel according to its nearest neighboring training samples in the feature space, and it has been widely used in satellite image classification [11].

For every classification process, a given ratio of pixels from each forest type was randomly selected as training samples, and the remaining pixels were used as testing samples. The total accuracy and the kappa coefficient were calculated for accuracy assessment. The ratio ranges from $0.1 \%$ to $5 \%$ in increments of $0.1 \%$, and we simulated ten times for every ratio in the original, rotation global, and rotation-K50 images. Various band compositions and $k$ from 3 to 6 were simulated, and their performance is similar (data not shown).

Fig. 6 shows one of the simulations with band compositions $7,5,4,3$, and 2 , and $k=5$ with the $k \mathrm{NN}$ classifier. They all exhibit stable kappa coefficient and total accuracy from a specific sample size onward. The proposed topographic normalized image yields the highest total accuracy and kappa coefficient with the initial smallest sample size. The image from the rotation global topographic normalization gives the worst simulation results. This indicates that the proposed method may help in distinguishing the forest types within large ranges of ICs, and it does not require a larger training sample size as compared with the other methods.

\section{Discussion And Conclusion}

\section{A. Novel Topographic Normalization Model}

Based on the performance analysis between the traditional rotation correction model and the new moving-window-based topographic normalization algorithm, this letter has suggested that the proposed model is a simple, more robust, and effective topographic normalization model. The proposed method is a band-specific topographic normalization model, and it was based on the hypothesis that the relationship between spectral features and ICs is site specific. Our results support this hypothesis and indicate that this approach is more accurate than those using global parameter estimation methods. More importantly, the proposed method does not require a priori parameter information; this indicates the generalizability of the algorithm. Although the final forest type classification map has not been given in this letter, the classification simulation comparison has demonstrated the limit of the global parameter estimation method and the potential of the local parameter estimation method in forest type classification at least.

\section{B. Opportunities for Development}

Some challenges remain unresolved. First, although the demonstrated robustness of the window size selection makes the image quality controllable, the optimization and evaluation of the window size selection are still needed. Second, the algorithm's computational demand is very high as the model executes all the steps of the global parameter estimation method pixel by pixel. Thus, the computing time linearly increases with the number of pixels in an image. The processing time has been $952.64 \mathrm{~s} /$ band of 1436 pixels $\times 1659$ pixels with an Intel Core i7-3520M central processing unit at $2.90 \mathrm{GHz}, 16-\mathrm{GB}$ random access memory, and 64-bit Windows 7 Professional operating system in this letter. Parallel computing design and further development could help solve this problem, or a more efficient and adaptive local parameter estimation method should be developed and evaluated for topographic normalization models. Third, to fully demonstrate generalizability, this site-specific approach needs further application to other models, such as the Minnaert or C-correction models, etc.

\section{ACKNOWLEDGMENT}

The authors would like to thank the project secretary Ms. S. Schreiner for her great project coordination; the project Ph.D. student X. Tang for the field work; and the Master students Ms. P. Wu and Ms. N. Lu from Anhui Agriculture University, Hefei, China, and Ms. C. Wu from the Chinese Academy of Forestry, Beijing, China, for the data collection. The authors would also like to thank the Shitai Forest Bureau for their very efficient cooperation and perfect logistical support.

\section{REFERENCES}

[1] D. Riaño, E. Chuvieco, J. Salas, and I. Aguado, "Assessment of different topographic corrections in Landsat-TM data for mapping vegetation types (2003)," IEEE Trans. Geosci. Remote Sens., vol. 41, no. 5, pp. 1056-1061, May 2003.

[2] H. Ge et al., "Pixel-based Minnaert correction method for reducing topographic effects on a Landsat 7 ETM+ image," Photogramm. Eng. Remote Sens., vol. 74, no. 11, pp. 1343-1350, Nov. 2008.

[3] B. Tan et al., "Improved forest change detection with terrain illumination corrected Landsat images," Remote Sens. Emviron., vol. 136, pp. 469-483, Sep. 2013.

[4] D. L. Civco, "Topographic normalization of Landsat Thematic Mapper digital imagery," Photogramm. Eng. Remote Sens., vol. 55, no. 9, pp. 1303-1309, Sep. 1989.

[5] S. Hantson and E. Chuvieco, "Evaluation of different topographic correction methods for Landsat imagery," Int. J. Appl. Earth Observ. Geoinf., vol. 13, no. 5, pp. 691-700, Oct. 2011.

[6] R. Richter, T. Kellenberger, and H. Kaufmann, "Comparison of topographic correction methods," Remote Sens., vol. 1, no. 3, pp. 184-196, Jul. 2009.

[7] M.-L. Gao et al., "Topographic correction of ZY-3 satellite images and its effects on estimation of shrub leaf biomass in mountainous areas," Remote Sens., vol. 6, no. 4, pp. 2745-2764, Mar. 2014.

[8] H. Reese and H. Olsson, "C-correction of optical satellite data over alpine vegetation areas: A comparison of sampling strategies for determining the empirical c-parameter," Remote Sens. Envinon., vol. 115, no. 6, pp. 1387-1400, Jun. 2011.

[9] Z. Szantoi and D. Simonetti, "Fast and robust topographic correction method for medium resolution satellite imagery using a stratified approach," IEEE J. Sel. Topics Appl. Earth Observ. Remote Sens., vol. 6, no. 4, pp. 1921-1933, Aug. 2013.

[10] M. Krzywinski and N. Altman, "Points of significance: Visualizing samples with box plots," Nat. Methods, vol. 11, no. 2, pp. 119-120, Feb. 2014.

[11] Q. Yu et al., "Object-based detailed vegetation classification with airborne high spatial resolution remote sensing imagery," Photogramm. Eng. Remote Sens., vol. 72, no. 7, pp. 799-811, Jul. 2006. 
Annex C: Manuscript III 


\title{
Effects of Intra-annual Illumination Conditions on NDVI in Mountainous Forest
}

\author{
Dengkui Mo ${ }^{1}$, , Lutz Fehrmann ${ }^{1}$, Hans Fuchs ${ }^{1}$, Paul Magdon ${ }^{1}$, Christoph Kleinn ${ }^{1 *}$ \\ ${ }^{1}$ Forest Inventory and Remote Sensing, Burckhardt Institute, Faculty of Forest Sciences and Forest Ecology, University of \\ Göttingen, Büsgenweg 5, 37077 Göttingen, Germany,dmo@gwdg.de, ckleinn@gwdg.de
}

\begin{abstract}
The normalized difference vegetation index (NDVI) derived from satellite-data has widely been used to support the assessment of status and changes of forest cover and forest biomass, and also in research studies for example on tree phenology. Many characteristics of the NDVI have been investigated, but the variability of NDVI caused by topography in steep mountain forests appears not to have obtained much attention. In these regions of high topographic variability, the intra-annual illumination (IL) conditions do considerably vary with the dynamics of solar-terrain-sensor geometry, and this affects the spectral response and derived vegetation indices. This study investigates the seasonal variability of NDVI in rough terrain forest. We carried out the statistical analysis by random sampling for all available Landsat 8 OLI images between May 2013 and October 2014 for our study area and examined if IL affects intra-annual NDVI on deciduous and coniferous forests. The findings indicate that IL and NDVI have a significant positive linear correlation; the slope coefficient of the linear functions shows a clear U-shape characteristic during a year. Meanwhile, we found a positive linear correlation between IL heterogeneity and variability of NDVI. Thus, the effects of illumination condition on NDVI or NDVI-related estimation should be taken into account in mountainous forest areas.
\end{abstract}

Keywords-illumination condition; Landsat 8 OLI; vegetation index; intra-annual variability; mountainous forest

\section{INTRODUCTION}

Vegetation indices are valuable remote sensing products for ecosystem monitoring and surface processes assessment, while the NDVI is certainly the most widely used [1]. Over the last two decades, NDVI and NDVI time-series have been applied directly to vegetation-related monitoring and assessment from regional [2] to global [3] scale. NDVI products derived from satellite images have been examined in many contexts of forest monitoring and forest research, including the assessment of forest cover change [4], forest aboveground biomass [5] and vegetation leaf phenology [6]. The NDVI can actually be considered a core variable for large area assessments of forest carbon stocks [7], forest biomass [8], and forest type mapping and the precision of the results is largely a function of the strength of the relationship between NDVI and these target variables.

As NDVI is a normalized ratio, it is widely assumed that it is not directly affected by illumination (IL) conditions. Lillesand et al. (2014) suggest that the NDVI helps compensate for image variations that are caused by changing illumination conditions as a function of slope and aspect [9]. Myeong et al. (2001) conclude that NDVI helps to mitigate the shadow effect of high-spatial resolution imagery and helps to improve the classification of vegetated areas [10]. However, systematic scientific studies that analyze if the IL variability can really be ignored when the NDVI is employed in mountainous forest areas are missing so far.

It is natural to assume that spectral variation caused by differences in the image acquisition geometry causes variability in NDVI. In the mountains, the radiometric variability of satellite images varies due to changing illumination conditions caused by the interaction between terrain, solar and sensor geometries [11]. In a given study site, the terrain condition can be assumed fixed, while the solar conditions for a given satellite sensor depend on the day of the year and on the time of the day of the image acquisitions.

In recent years, a variety of topographic normalization (or correction) methods have been developed to reduce spectral variability caused by topography and to improve the accuracy of forest type classification[12], forest change detection [13], and fractional tree cover mapping in tropical mountains [14]. These studies have compared a variety of topographic normalization models under different conditions, but the performance of these models was found to be highly sitespecific. However, it is still unclear whether there is a universal and optimal topographic normalization model that can considerably reduce or even eliminate the variation caused by IL variability. Further, these studies did not completely address how IL affects NDVI.

Thus, the possible interactions between IL and NDVI time series and how these can be used to explain over- or underestimations of NDVI needs to be clarified; this will then also have an impact on related estimates of forest biomass or carbon stocks.

In this study, we developed such a generalized model where we assess the impact of intra-annual IL on NDVI time series directly in a mountain forest using stratified sampling and statistical analysis.

\section{MATERIAL AND METHODS}

\section{A. Study area}

The study area is a subtropical mountain forest area in midlatitudes, located in Shitai County, Anhui Province, in the Southeast of China and has an area of $1412 \mathrm{~km}^{2}$. Major parts of the study site are characterized by steep slopes and a rugged topography, with elevations ranging from about 10 to $1719 \mathrm{~m}$ (Figure 1). $81.39 \%$ of the area is covered by deciduous and evergreen forests, including large plantation areas (Latest data 
published in shitai County Forestry Bureau: http://st.ahlgw.cn/SortHtml/618/82501198637.html; Chinese, accessed 07/12/2017). Because of its high forest cover and low population density, Shitai County is mainly developing the tourism and tea industry. Forest management is at low intensity, and land use patterns are relatively stable.

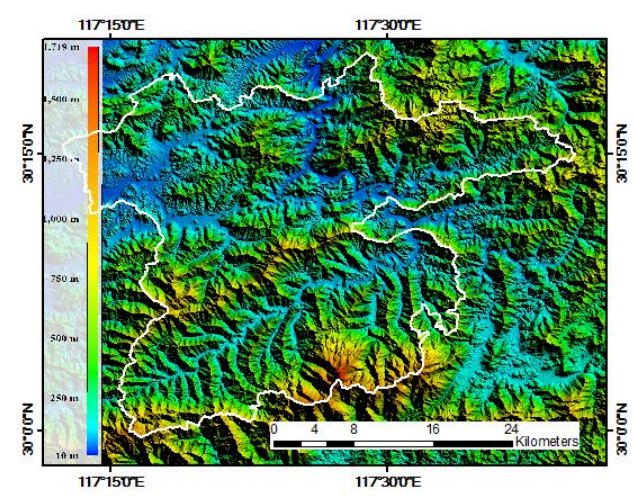

Figure 1. The study site and illustration of terrain conditions produced from SRTM 1 Arc-Second Global simulated as shaded relief with sun elevation $=60^{\circ}$, and sun azimuth $=45^{\circ}$.

\section{B. Data collection}

We assume that the heterogeneity of local terrain and illumination cannot be neglected for medium and high spatial resolution optical remotely sensed imagery such as Landsat and Sentinel-2. We used a total of 11 cloud-free Landsat 8 Operational Land Imager (OLI) images for this study, which are located in the Landsat Worldwide Reference System-2 (WRS2) scene path 121 and row 39. Due to the absence of cloud-free images, we needed to pool two consecutive years (2012-2013) of Landsat 8 OLI images to cover four seasons throughout the year assuming that changes in NDVI are negligible and seasonality is comparable in both years. Details of the Landsat 8 OLI images are given in Figure 2. Hereafter, the images are sorted by their Julian date (day of the year, DOY).

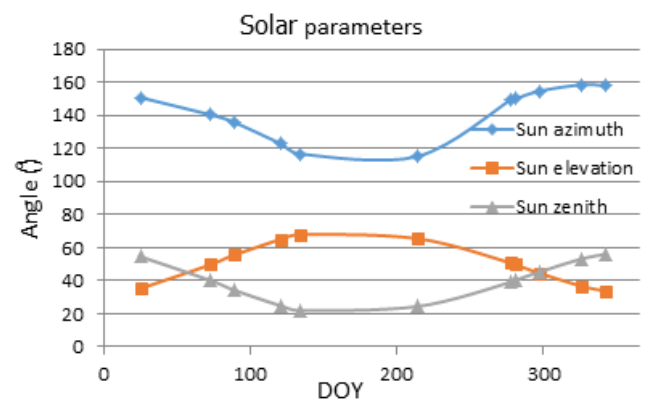

Figure 2. Variation in solar parameters of cloud-free Landsat 8 OLI time series.

Data of the Shuttle Radar Topography Mission (SRTM) 1 Arc-Second Global was used to create the terrain model of the study area because its spatial resolution is relatively close to that of Landsat 8 optical bands and it has been successfully applied in recent studies [14]. We downloaded Landsat 8 OLI Surface Reflectance (SR) data and SRTM 1 Arc-Second Global images from the United States Geological Survey
(USGS) Earth $\quad$ Explorer $\quad$ Platform (https://earthexplorer.usgs.gov; accessed 07/12/2017). This digital elevation model (DEM) was projected and resampled to the same projection and pixel size with Landsat 8 OLI images using bilinear interpolation.

We produced a forest type cover map from multi-temporal Landsat 8 OLI images, where three forest types were distinguished: deciduous (DCD), evergreen (EVG), and bamboo forest. Furthermore, we restricted the analysis to mountain forest by filtering according to slope conditions $\left(\geq 5^{\circ}\right)$. Bamboo forests were excluded from this study, because their coverage is relatively low and they occur in scattered small patches only. Random samples taken from DCD and EVG classes were visually verified using a combination of multitemporal RapidEye composites and Google Earth imagery.

\section{Illumination condition and NDVI time series}

It is well known that illumination condition can be derived from terrain and solar parameters [13, 15-18]. IL time-series can be simulated using a terrain model and the intra-annual solar parameters obtained from the metadata of satellite images. In this study, synchronized illumination condition data and NDVI time series were generated to explore the interaction of NDVI and IL during the year. We assume, of course, that the terrain conditions of the study site remain constant for two consecutive years. Here, the slope and aspect data were created from topographic modeling on SRTM 1 Arc-Second Global data with a kernel size of 5 by 5 pixels.

To simulate the illumination conditions for a given DOY, we used the satellite parameters at the point for the given acquisition time; from these we calculated the IL conditions for each pixel using the formula (1), following [13].

$$
\mathrm{IL}=\cos (Z) \cos (S)+\sin (Z) \sin (S) \cos \left(\varphi_{z}-\varphi_{S}\right)
$$

Here, $Z$ is the solar zenith angle and $\varphi_{Z}$ is the solar azimuth angle. These two solar parameters are approximately constant over the entire image and can directly be read from the metadata file of Landsat 8 OLI data. $S$ is the topographic slope angle and $\varphi_{S}$ is the aspect angle of the topographic surface. The value of IL ranges from -1 to 1 with $\mathrm{IL}=0$ for a horizontal surface.

We used pre-selected cloudless Landsat 8 OLI SR data to compute NDVI for a given DOY directly. For each Landsat 8 OLI SR data, NDVI was calculated from the red and nearinfrared surface reflectance [1] along

$$
\mathrm{NDVI}=\frac{\rho_{\text {nir }}-\rho_{\text {red }}}{\rho_{\text {nir }}+\rho_{\text {red }}}
$$

where $\rho_{\text {red }}$ and $\rho_{\text {nir }}$ are surface reflectance in the red and near-infrared band, respectively. Finally, both IL and NDVI time series are sorted in ascending order according to the DOY. 


\section{Sampling approach}

For each pixel, topographic features are stable over the period of this study, but the illumination conditions vary as a function of the point in time of image acquisition. For a given pixel, the forest type may change within the two years study period due to forest logging, forest fires or other land cover changes. We excluded these pixels from our analyses to reduce confounding effects introduced by land cover changes because we were merely interested in the effect of variation in IL. To obtain illumination conditions for deciduous and evergreen forests in a systematic manner, we used a sampling method to select a set of locations from different topographic characteristics groups. The groups are defined as follows.

Slope $\left({ }^{\circ}\right)$ : 5-10, 10-20, 20-30, 30-40, 40+ (5 groups)

Aspect $\left({ }^{\circ}\right): 0-45,45-90,90-135 \ldots$ 315-360 (8 groups)

Elevation (m): 0-300, 300-600,600-900, 900+ (4 groups)

This breakdown the three variables slope, aspect, and elevation with 5,8 and 4 levels respectively, which results in $5 * 8 * 4=160$ combinations in total. We first randomly selected 10 samples from each combination, a total of 1600 samples. For better visualization of trends in the subsequent figures, we took a random subsample of 250 points out of these 1600 . The coordinates of these 250 sample pixels were used for all 11 pairs of values of NDVI and IL. Finally, the NDVI and IL values were determined for the fixed coordinates selected above. In one of the random sampling cases, 100 DCD and 150 EVG locations were randomly selected. Hereafter, all statistical analyses are based on this sample.

\section{E. Analysis}

We explored the linear correlation between values of NDVI and of IL per DOY and their response patterns between intraannual NDVI and IL time series.

As the first step of the analysis, simple linear regression [19] was used to analyze the type of relationship between NDVI and IL for the given forest types separately, in which larger values of NDVI are associated with larger values of IL (increasing trend) or smaller values of IL (decreasing trend). The Goodness-of-Fit was assessed by comparing the $\mathrm{R}^{2}$ and significance of the linear regression between IL and NDVI from different DOYs.

Compared with linear regression, non-linear regression requires a larger sample size. As the second step of the analysis, a non-linear regression also analyzes the trend of the slope coefficients over the DOY for both forest types together. Therefore, we analyzed the non-linear regression between slope coefficients and DOY using forest samples.

Further, we analyzed correlation and linear regression between IL variability and NDVI variability. We used the coefficient of variation $(\mathrm{CV})$ as a relative measure of variability. $\mathrm{CV}$ is the ratio of the standard deviation to the mean. The mode fit was evaluated based on the coefficient of determination $\left(\mathrm{R}^{2}\right)$ and statistical significance (p-value). Although the linear model does not necessarily provide the optimal model fit for predicting $\mathrm{CV}$ of NDVI, it was considered appropriate for testing the effect of CV of IL.

\section{RESULTS}

Scatter plots and linear regression lines between IL and NDVI for each DOY are shown in Figure 3. In general, the NDVI of the evergreen forest is higher than that of the deciduous forest in early stages of leaf growth and leaf-off conditions. IL and NDVI are positively correlated for all the DOYs in both deciduous and evergreen forests, and these positive correlations are significant ( $\mathrm{p}$-value $<0.001$ ).

The linear regression between IL and NDVI shows a stronger relationship (higher $\mathrm{R}^{2}$ values) with NDVI at the beginning and end of the year than in the middle of the year for the evergreen forest with few phonological differences. The weakest relationships were observed in (DOY121, DOY134, and DOY214), although the relationships were still significant (p-value $<0.001$ ). When jointly analyzing Figures 2 and 3 , one sees that the three weakest relationships correspond directly to the three highest solar elevation angles. We, therefore, conclude that these acquisitions are least affected by topographic illumination effects.

For the deciduous forest with higher phenological variability, the linear regression between IL and NDVI shows a strong relationship (higher $\mathrm{R}^{2}$ values) between IL and NDVI for most DOYs (the periods with fully leaf-on or fully leafoff). The three minimum $\mathrm{R}^{2}$ values correspond to three DOYs (DOY73, DOY89, and DOY121, from 14-Mar-14 to 1-May14), which are in the leaf-off. Similar to evergreen forests, the stronger relationship occurred at the beginning and end of the year than that in the middle of the year.

We found an apparent characteristic that the slope coefficients of the linear regression are first decreasing and then increasing in the year for both forest types, as depicted in Figure 3. After having obtained these results, we modeled the relationship between the slope coefficients and DOYs using a second-degree polynomial function. A U-shaped curve was built in Figure 4 . The correlation coefficient $\left(R^{2}\right)$ for the regression curve for the slope coefficients vs. DOYs is 0.914 , which indicates a good fit of the curve. Similar to the slope coefficients, the ranges of IL conditions are first decreasing and then increasing in the year (see Figure 3). Thus, steeper slopes mean higher differences of NDVI at the beginning and end of the year than that in the middle of the year.

We used the coefficients of variation (CVs) to express the magnitude of intra-annual variability in NDVI and IL conditions. Figure 5 shows significant positive correlations between CVs of IL and CVs of NDVI in both deciduous and evergreen forests. 

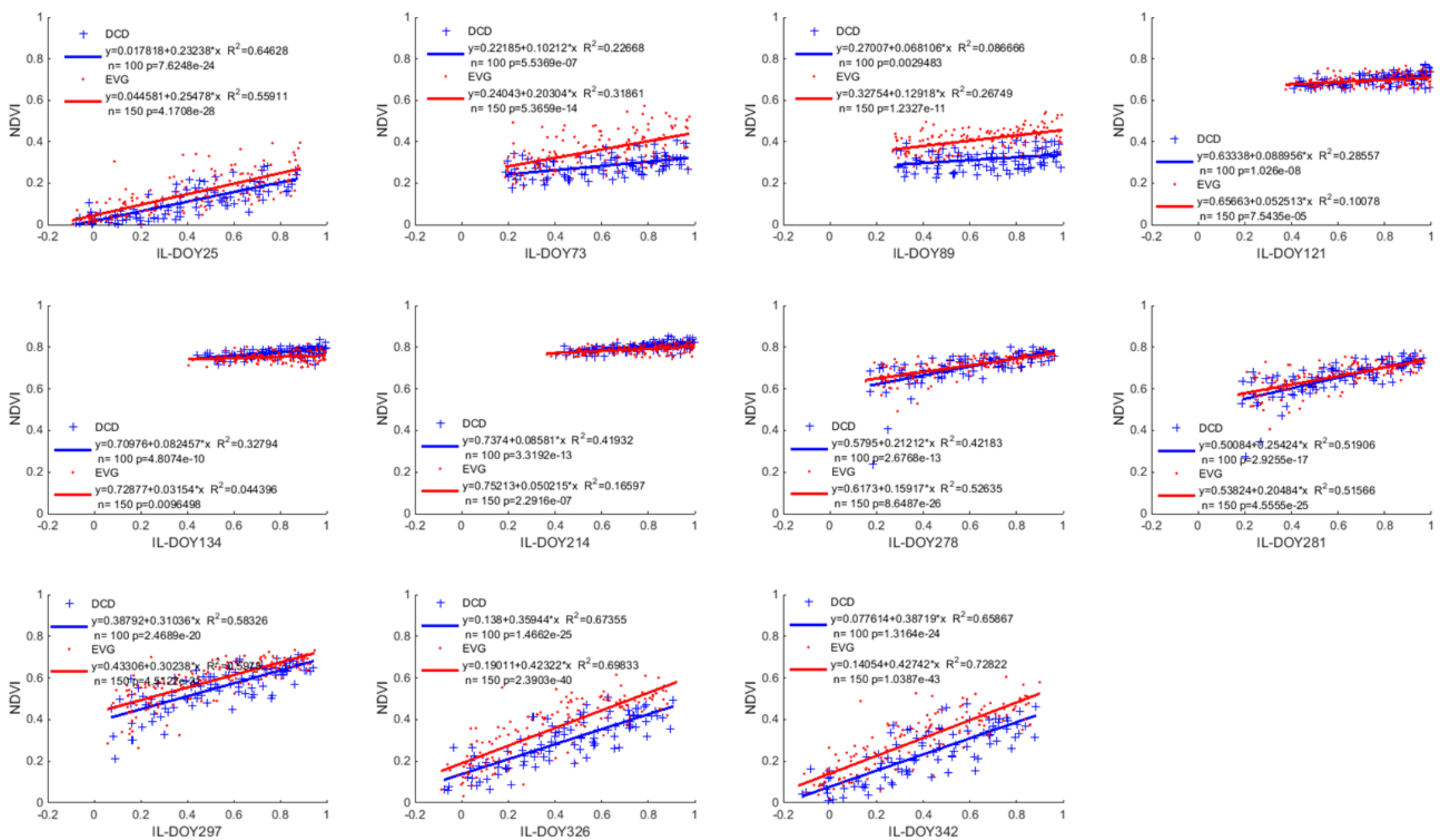

Figure 3. Scatter plots and linear regression lines of NDVI over IL per DOY during the study period from DOY 25 to DOY 342 . All p-value $<0.001$. DCD $=$ deciduous forest; $\mathrm{EVG}=$ evergreen forest; $\mathrm{DOY}=$ the day of the year. Sample size for $\mathrm{DCD}=100$, for $\mathrm{EVF}=150$ 


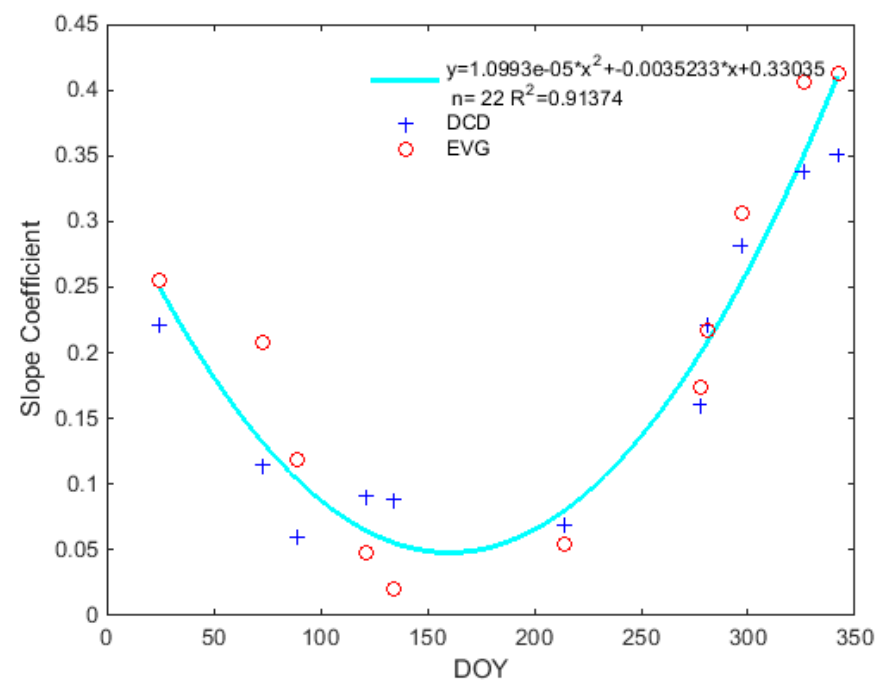

Figure 4. Polynomial curve fitting for the slope coefficients vs. DOYs, data from the deciduous and evergreen forest were jointly analyzed for this model adjustment. Sample size $=22$.

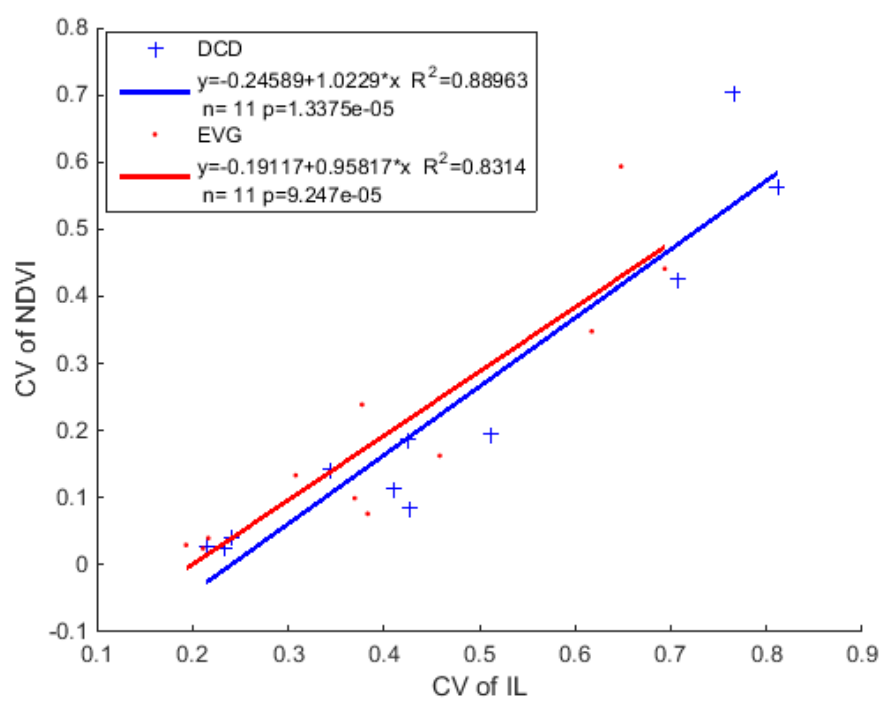

Figure 5. Scatter plots and linear regression showing the relationship between the coefficients of variation (CV) of IL and the CV of NDVI for deciduous forest and evergreen forest separately.

\section{DISCUSSION AND CONCLUSIONS}

In this case study, we directly analyzed the effects of intra-annual illumination conditions on NDVI using sampling statistics from a cloud-free Landsat 8 OLI time series in a mid-latitude mountain forest with steep slopes samples from deciduous and evergreen forests are employed for statistical analysis. We found that NDVI had a strong linear relationship with IL at the beginning and end of the year with low solar elevation angle. Three lowest correlation coefficients between IL and NDVI correspond to three DOYs (DOY73, DOY89, and DOY121, from 14Mar-14 to 1-May-14) for the deciduous forest. Probably because diverse deciduous species have a variety of leaf-off phenology characteristics, the heterogeneity of NDVI during this period is high resulting in a weak correlation between IL and NDVI.

Within a year, the slope coefficient of the linear regression between IL and NDVI has a clear "U" shape. According to Figure 4, we can conclude that the corresponding DOY of its lowest point of the curve would be the best acquisition date to reduce the topographic illumination effect, which corresponds to the highest sun elevation. 
The heterogeneity of NDVI has a significant linear positive correlation with IL variability in this study area. Since IL and its variability can be calculated in advance, the variability of NDVI can be predicted under the given terrain model and the solar parameters. Thus, illumination condition is a factor of NDVI variability in the seasonal analysis especially when the heterogeneity of IL is high, and the effects of illumination conditions on NDVI or NDVI related estimation should not be neglected in mountain forests.

It is worth mentioning that uncertainty may also come from the aggregated NDVI time series because NDVI varies with the growth and decline of the forest during two years. In order to get more NDVI time series in one year, satellite data with higher temporal resolution (such as Sentinel-2 or Planet) should be tested. Besides, more vegetation indices such as Enhanced Vegetation index (EVI), Soil-Adjusted Vegetation Index (SAVI), etc. should be studied with their intra-annual IL time series.

Some studies have tried to analyze and remove the effects of illumination condition on NDVI using some topographic correction models [14, 20,21], but the conclusions are quite different. Valerianoet al. (2016) concluded that the topographic correction was required for a consistent reduction of the topographic effect on the vegetation indices including NDVI from rugged terrain [20]. However, in other studies, NDVI was considered insensitive to the topography as it is a ratio of two bands [22] or NDVI was less sensitive to terrain illumination than the enhanced vegetation index (EVI) [21]. We cannot conclude that comparisons before and after topographic correction may be directly used to evaluate whether IL significantly affects NDVI or these studies are site-specific.

A recent literature [14] has pointed out that NDVI was not affected by topographical effects and related illumination conditions, and topographical normalization did not improve fractional tree cover mapping in tropical mountains. Maybe due to its relatively high solar elevation (from 50 to 64 degree) in this tropical region [14], the effect of IL on NDVI is relatively low. In our study site of the middle latitudes, IL effects on NDVI are strong especially when the solar elevation angle is relatively low. Therefore, we may have reason to conclude that the effect of IL on NDVI is stronger at higher altitudes in mountainous areas and further studies should be extended to larger geographical scales (such as regional and global scales).

\section{ACKNOWLEDGMENT}

This research was jointly supported by the German Federal Ministry of Education and Research (BMBF), (Project Lin4Carbon Grant No. 033L049C), the Chinese Academy of Forestry (CAF) (Grant No. Lin2Value CAFYBB2012013), and the National Natural Science Foundation of China (Grant No. 31470643 and 31100412).

\section{REFERENCES}

[1] Y. Ke, J. Im, J. Lee, H. Gong, and Y. Ryu, "Characteristics of Landsat 8 OLI-derived NDVI by comparison with multiple satellite sensors and in-situ observations," Remote Sensing of Environment, vol. 164, pp. 298$313,7 / / 2015$.

[2] N. Zeng, J. D. Neelin, K.-M. Lau, and C. J. Tucker, "Enhancement of interdecadal climate variability in the Sahel by vegetation interaction," Science, vol. 286, no. 5444, pp. 1537-1540, 1999.

[3] Z. Zhu et al., "Greening of the Earth and its drivers," Nature climate change, vol. 6, no. 8, pp. 791-795, 2016.

[4] M. C. Hansen et al., "High-Resolution Global Maps of 21st-Century Forest Cover Change," Science, vol. 342, no. 6160, pp. 850-853, 2013-11-15 00:00:00 2013.

[5] X. Zhu and D. Liu, "Improving forest aboveground biomass estimation using seasonal Landsat NDVI timeseries," ISPRS Journal of Photogrammetry and Remote Sensing, vol. 102, pp. 222-231, 2015.

[6] R. Buitenwerf, L. Rose, and S. I. Higgins, "Three decades of multi-dimensional change in global leaf phenology," Nature Clim. Change, Letter vol. 5, no. 4, pp. 364-368, 04//print 2015.

[7] K. Tan, S. Piao, C. Peng, and J. Fang, "Satellite-based estimation of biomass carbon stocks for northeast China's forests between 1982 and 1999," Forest ecology and management, vol. 240, no. 1, pp. 114-121, 2007.

[8] H. Riihimäki, J. Heiskanen, and M. Luoto, "The effect of topography on arctic-alpine aboveground biomass and NDVI patterns," International Journal of Applied Earth Observation and Geoinformation, vol. 56, pp. 44-53, 2017.

[9] T. Lillesand, R. W. Kiefer, and J. Chipman, Remote sensing and image interpretation. John Wiley \& Sons, 2014.

[10] S. Myeong, D. J. Nowak, P. F. Hopkins, and R. H. Brock, "Urban cover mapping using digital, high-spatial resolution aerial imagery," Urban Ecosystems, vol. 5, no. 4, pp. 243-256, 2001. 
[11] H. Li, L. Xu, H. Shen, and L. Zhang, "A general variational framework considering cast shadows for the topographic correction of remote sensing imagery," ISPRS Journal of Photogrammetry and Remote Sensing, vol. 117, pp. 161-171, 2016.

[12] D. Mo, H. Fuchs, L. Fehrmann, H. Yang, Y. Lu, and C. Kleinn, "Local Parameter Estimation of Topographic Normalization for Forest Type Classification," Geoscience and Remote Sensing Letters, IEEE, vol. PP, no. 99, pp. 1-5, 2015.

[13] B. Tan et al., "Improved forest change detection with terrain illumination corrected Landsat images," Remote Sensing of Environment, vol. 136, pp. 469-483, 2013.

[14] H. Adhikari, J. Heiskanen, E. E. Maeda, and P. K. Pellikka, "The effect of topographic normalization on fractional tree cover mapping in tropical mountains: An assessment based on seasonal Landsat time series," International Journal of Applied Earth Observation and Geoinformation, vol. 52, pp. 20-31, 2016.

[15] D. Riaño, E. Chuvieco, J. Salas, and I. Aguado, "Assessment of different topographic corrections in Landsat-TM data for mapping vegetation types (2003)," IEEE Transactions on geoscience and remote sensing, vol. 41, no. 5, pp. 1056-1061, 2003.

[16] H. Ge, D. Lu, S. He, A. Xu, G. Zhou, and H. Du, "Pixel-based Minnaert correction method for reducing topographic effects on a Landsat 7 ETM+ image," Photogrammetric Engineering \& Remote Sensing, vol. 74, no. 11, pp. 1343-1350, 2008.

[17] D. L. Civco, "Topographic normalization of Landsat Thematic Mapper digital imagery," Photogrammetric Engineering and Remote Sensing, vol. 55, no. 9, pp. 1303-1309, 1989.

[18] D. Mo, H. Fuchs, L. Fehrmann, H. Yang, C. Kleinn, and Y. Lu, "Moving window-based topographic normalization of optical satellite imagery for forest mapping in mountainous terrain," in Earth Observation and Remote Sensing Applications (EORSA), 2014 3rd International Workshop on, 2014, pp. 452-456: IEEE.

[19] N. Altman and M. Krzywinski, "Points of Significance: Simple linear regression," Nat Meth, This Month vol. 12, no. 11, pp. 999-1000, 11//print 2015.

[20] M. d. M. Valeriano, I. D. A. Sanches, and A. R. Formaggio, "TOPOGRAPHIC EFFECT ON SPECTRAL VEGETATION INDICES FROM LANDSAT TM DATA: IS TOPOGRAPHIC CORRECTION NECESSARY?," Boletim de Ciências Geodésicas, vol. 22, no. 1, pp. 95-107, 2016.

[21] L. S. Galvão, F. M. Breunig, T. S. Teles, W. Gaida, and R. Balbinot, "Investigation of terrain illumination effects on vegetation indices and VI-derived phenological metrics in subtropical deciduous forests," GIScience \& Remote Sensing, vol. 53, no. 3, pp. 360-381, 2016.

[22] A. Banskota, N. Kayastha, M. J. Falkowski, M. A. Wulder, R. E. Froese, and J. C. White, "Forest monitoring using Landsat time series data: a review," Canadian Journal of Remote Sensing, vol. 40, no. 5, pp. 362-384, 2014. 
Annex D: Manuscript IV 
Article

\title{
A Simple and Robust Haze Removal Method for VHR Optical Satellite Image Based on Mean Vector $L^{2}$-norm
}

\author{
Dengkui Mo ${ }^{1,2,3 *}$, Hans Fuchs' ${ }^{1}$ Lutz Fehrmann" ${ }^{1}$, Nils Nölke', Paul Magdon ${ }^{1}$, Christoph Kleinnn ${ }^{1, *}$ \\ 1 Forest Inventory and Remote Sensing, Faculty of Forest Sciences and Forest Ecology, Georg-August-Universität \\ Göttingen, Büsgenweg 5, 37077 Göttingen, Germany \\ 2 Research Center of Forestry Remote Sensing \& Information Engineering, Central South University of Forestry \\ and Technology, Changsha 410004, China \\ 3 Key Laboratory of Forestry Remote Sensing Based Big Data \& Ecological Security for Hunan Province, Changsha \\ 410004, China \\ * Correspondence: dmo@gwdg.de; CKleinn@gwdg.de; Tel.: +49 [0]551 39-33472 \\ Academic Editor: name
}

Received: date; Accepted: date; Published: date

\begin{abstract}
Multispectral remotely sensed images often suffer from haze degradation, which reduces visual interpretability and affects further image analysis processes. Hence, haze detection and removal techniques are essential for multispectral image pre-processing. Haze detection from very high spatial resolution (VHR) satellite images based on a haze thickness map (HTM) is challenging in cases where large bright objects can be confused with haze. This paper proposes a new simple and robust haze detection and removal method for VHR multispectral remotely sensed images. Different from existing HTM-based methods, the proposed method estimates the HTM from the mean vector $\mathrm{L}^{2}$-norm of the blue band in a given local sample window. Moreover, we improved the compensation strategy for both hazefree and haze pixels. The proposed method was successfully applied to a variety of VHR optical satellite images with complex haze cover over densely built-up regions.
\end{abstract}

Keywords: L2-norm; haze thickness map; haze detection; haze removal; Worldview 3 satellite image; very high-resolution satellite imagery

\section{Introduction}

While acquiring imagery from satellites or aircrafts, different characteristics of the atmosphere can lead to degradations of image quality and visual interpretability. Water vapor and water droplets, smoke, dust, and aerosols are all considered "haze" due to their similar effect in reducing the image quality by scattering and spectral distortion in the visible spectral bands [1] which significantly compromises useful analysis. Contrary to cloud cover in images, a 
haze affected image transparency can be compensated to a certain extent by image restoration techniques. Thus, there remains a practical need for a scene-based, simple-to-use and robust haze removal method for very high spatial resolution (VHR, ground sampling distance (GSD) $<=5 \mathrm{~m}$ ) optical satellite imagery to improve further image analyses including classification. As completely haze-free satellite images of the earth's surface are usually rare, some studies focus on haze removal based on single images, and we briefly summarize the corresponding state-ofthe-art approaches.

Most of the studies focus on de-hazing methods using terrestrial outdoor photos. Fattal [2] and Tan [3] have proposed the earliest algorithms for single image haze removal. Recently, the most applied method for single image haze removal is the dark channel prior (DCP) method developed by $\mathrm{He}$ et al. [4]. Some improved algorithms [5-8] were proposed to overcome weaknesses of the DCP method. These approaches are mainly inspired by the widely used dark-object subtraction (DOS) technique [9] and are based on the assumption that dark channel intensities of outdoor photos are very low and close to zero. The dark channel is calculated from the minimum value of the neighborhood pixels using a haze sensitive band from a given window.

Long, Shi et al. [10] and Pan, Xie et al. [11] manually selected 1000-5000 haze-free remote sensing images from Google Earth RGB screenshots. The statistics indicate that the average intensity of Google Earth images' dark channel is low, but not close to zero. Thus, Pan, Xie et al. [12] introduced a new translation term for the DCP method, as the difference in dark channels between Google Earth images and outdoor photos is significant and cannot be neglected. As Google Earth's tiles have been processed by image combinations and image enhancement, the pixel values are stretched to a range between 0 and 255. According to the statistical analysis, the translation term of digital numbers (DN), radiance or reflectance of satellite images cannot be defined in advance. In addition, the DCP method assumes that in atmosphere transmission models, the scene radiance is attenuated exponentially with the scene depth (the distance between an object and the observer) for terrestrial outdoor photos [1,4]. In a satellite image, the scene depth is almost constant, and maybe that is why this category of methods has not been widely proven effective.

As mentioned above, satellite images are different from terrestrial outdoor photos. Satellite image de-hazing methods aim to recover the surface reflectance by correcting haze pixels based on the imaging model. Dark-object subtraction (DOC) [9] and haze optimized transformation (HOT) [13] techniques are well-known de-hazing methods for visual satellite images. Chavez [9] proposed an approach called additive model or dark-object subtraction (DOS) technique in 1988 and Zhang et al. [13] proposed HOT to detect and characterize the spatial distribution of haze/cloud in Landsat scenes. Makarau et al. [14,15] present a further development of the DOS technique constructing a HTM based on a local sample window from the whole image and propose a subtraction of the HTM from a hazy image to restore the haze-free image. ATCOR 2/3 was initially developed by Schläpfer and Richter [14-18] and considered as the state-of-the-art atmospheric and topographic correction for optical satellite images. The major challenge is to 
construct a more precise HTM considering the disturbing influence of bright ground objects, especially for VHR satellite imagery with a complex haze over densely built-up areas.

Inspired by the idea proposed by Makarau et al. [14,15], we present a simple and robust haze removal method for VHR satellite imagery. We assume that haze in satellite images is locally uniform and can be distinguished from the background value and bright objects. We use a mean vector $\mathrm{L}^{2}$-norm of the blue band to estimate the HTM ( $\mathrm{L}^{2}$-norm HTM). The proposed $\mathrm{L}^{2}-$ norm HTM is the most important step and the main contribution of this method. The mean vector $\mathrm{L}^{2}$-norm of the blue band helps to stabilize noise, shadow, and dark object pixels while preserving edge structural features and enhancing the contrast of haze, bright objects, and background colors. We also improve HTM refinement and compensation strategy for both haze-free and haze pixels. The mean differences between the haze pixels and the haze-free pixels are compensated according to the relative brightness to the red band.

\section{Methodology}

\subsection{General haze imaging model}

Referring to $[9,14,15]$, a general haze imaging model for optical satellite data can be expressed as follows:

$$
D N_{i}(x, y)=D N_{i}^{\text {radiation }}(x, y)+H T M_{i}(x, y)
$$

Where $D N_{i}(x, y)$ is the acquired digital number $(\mathrm{DN})$ for band $i$ of a pixel with image

coordinates $(x, y), D N_{i}^{\text {radiation }}(x, y)$ is the DN without the influence of haze, $\operatorname{HTM}_{i}(x, y)$ is the estimated haze thickness of the pixel for the band $i$.

As the influence of haze decreases with increasing wavelength, $\mathrm{HTM}_{\mathrm{i}}$ can be derived from $\operatorname{HTM}(x, y)$ of the most haze-sensitive band. In general, most VHR optical satellite images include at least four bands (infrared, red, green, blue), where the blue band is most sensitive to haze. The haze thickness map $\operatorname{HTM}_{i}(x, y)$ can be estimated from the blue band by equation (2):

$$
\operatorname{HTM}_{i}(x, y)=a_{i} \times \operatorname{HTM}_{\text {blue }}(x, y)+b_{i}
$$

where $a_{i}$ is the band-dependent slope coefficient and $b_{i}$ is the band-dependent intercept of the linear regression of $\operatorname{HTM}_{i}(x, y)$ versus $H T M_{\text {blue }}(x, y)$. In general, $H_{T M}$ blue refers to HTM in other references.

We assume that $a_{i}$ and $b_{i}$ are constant for one image, and we get (3) from (1) and (2) for a VHR satellite image

$$
D N_{i}^{\text {radiation }}=D N_{i}-a_{i} \times H T M_{\text {blue }}-b_{i}
$$


where $D N_{i}^{\text {radiation }}$ is the haze-free image, DN is the band $i$ image with the haze effects, and HTM is the haze thickness map of the blue band image. The precision of the HTM directly influences the quality of the de-hazing model. In addition, a typical overcorrection of haze-free pixels (considering variables in the equation (2) and (3)) should be compensated.

\subsection{Established methods for HTM estimation}

According to Makarau et al. (2014) [14] estimating the HTM is done by searching the darkest pixels using a moving window in the whole haze-sensitive band (for the blue band using equation (4)) followed by resampling to the original image spatial resolution using a nearest neighbor interpolation.

$$
\operatorname{HTM}_{\text {blue }}(x, y)=\min _{\left(x^{\prime}, y^{\prime}\right) \in W(x, y)}\left(\operatorname{Blue}\left(x^{\prime}, y^{\prime}\right)\right)
$$

Where $W(x, y)$ is a moving window, centered at $(x, y), w$ is the size of the window, $\operatorname{Blue}(x, y)$ is the pixel value of blue band which is most affected by haze.

In theory, the standard HTM only includes background pixels and haze pixels. Thus dark objects lead to an underestimation in the HTM, and bright objects result in overestimation. To correct the HTM, refinement methods suggested by [14,15] use two strategies as follows:

To avoid underestimation in the HTM, the HTM according to Makarau et al. (2014) [14] is calculated using the synthetic and extrapolated band obtaining higher thickness values than the original band. However, the effect of this method is not obvious for VHR satellite images over dense built-up regions because of a large number of small shadow areas.

To avoid overestimation of the HTM, bright objects are masked using a threshold segmentation of the red band, and then these masked pixels are interpolated for HTM. In practice, parameters for image segmentation need to be determined in advance, and image segmentation is a process with large computation cost generally. Further, one single threshold might not be useful for both haze and haze-free regions.

Thus, we propose a new HTM estimation and refinement method, which we refer to as "mean vector L²-norm HTM method".

\subsection{Mean vector $L^{2}$-Norm HTM Method}

Under an extreme condition, a very dark pixel in the haze region causes a haze underestimation. Haze pixels that are close to a dark pixel in the window would be identified as non-haze area (see Figure 1.). Once these haze pixels cannot be detected, they naturally cannot be removed. Labeling these pixels and avoiding underestimation is a challenge in practice. We assume that replacing pixels with the mean vector $\mathrm{L}^{2}$-norm value can help solving the HTM underestimation problem and will not significantly affect the haze thickness estimation if the haze is locally homogeneous. In addition, the $\mathrm{L}^{2}$-norm based HTM may be more robust and free

of block-effects. Jha et al. (2016) proposed and validated the excellent characteristics of this 
technique for haze removal from single images [19]. Therefore, we calculate the HTM from the mean vector $L^{2}$ - norm of the haze-sensitive band instead of the original band.

\begin{tabular}{|l|l|l|l|l}
\hline 5 & 5 & 5 & 5 & 5 \\
\hline 5 & 5 & 5 & 5 & 5 \\
\hline 5 & 5 & 0 & 5 & 5 \\
\hline 5 & 5 & 5 & 5 & 5 \\
\hline 5 & 5 & 5 & 5 & 5 \\
\hline
\end{tabular}$\longrightarrow$\begin{tabular}{|l|l|l|l|l|}
\hline 0 & 0 & 0 & 0 & 0 \\
\hline 0 & 0 & 0 & 0 & 0 \\
\hline 0 & 0 & 0 & 0 & 0 \\
\hline 0 & 0 & 0 & 0 & 0 \\
\hline 0 & 0 & 0 & 0 & 0 \\
\hline
\end{tabular}

Figure 1. A schematic example of HTM underestimation. Left: Simulated haze-sensitive image, right: HTM of the left image. " 5 " represents haze, " 0 " represents the dark object, and the size of the window is 5 by 5 pixels. Shadow pixels are easily found around trees, buildings, etc., especially in VHR optical satellite data. Meanwhile, shadow pixels can cause all haze pixels in the window cannot be detected and HTM underestimation.

We calculate the $\mathrm{L}^{2}$-norm of the intensities of the pixels $x_{i} \in \Omega(x)$ and divide this L2-norm value by the number of pixels present in a window to get the mean vector intensity value for each window. This mean vector value is assigned to the pixel. If the blue band is used as the haze-sensitive image, the mean vector $\mathrm{L}^{2}$-norm blue image is calculated from the equation (5).

$$
\overline{\text { Blue_L } \mathrm{L}^{2}}:=\sqrt{\frac{1}{N} \sum_{i=1}^{N}{ }_{x_{i} \in \Omega(x)} x_{i}^{2}}
$$

Where $\mathrm{N}$ is the number of pixel in a given overlapping window $\Omega(x)$, the window size is $\mathrm{w}$, and $N=w^{*} w$. Since we have taken the $\mathrm{L}^{2}$-norm over the intensities of neighboring pixels in the local window, $\overline{B l u e_{-} L^{2}}$ depends equally on every pixel and is less affected by the intensity of any particular pixel $x_{i} \in \Omega(x)$. Thus, $\overline{B l u e_{-} \mathrm{L}^{2}}$ is more robust as compared to the original blue band. Moreover, for large window size - that is, for a sufficiently large $\mathrm{N}$

$$
\overline{\text { Blue_L } \mathrm{L}_{N \rightarrow \infty}^{2}} \rightarrow \text { background_value }
$$

The mean vector $\mathrm{L}^{2}$-norm image helps to distinguish background, haze veil and bright objects (see Figure 2, the location and data source refer to Figure 4). According to visual inspections, the haze thickness has the same spatial distribution and a stable range with increasing w. Meanwhile, the bright objects can be distinguished readily from the background value and haze using mean vector L2-norm of the blue band. As we increase window size w, $\overline{\text { Blue _ } \mathrm{L}^{2}}$ is very close to the background (all the pixels have the same value, can be used as background value). However, the computation costs significantly increase with larger $w$. Therefore, we use $w=8$ (GSD is 1.2 meters in Worldview 3 multispectral image, 8 pixels equal approximatively 10 meters on the ground) in this paper. 


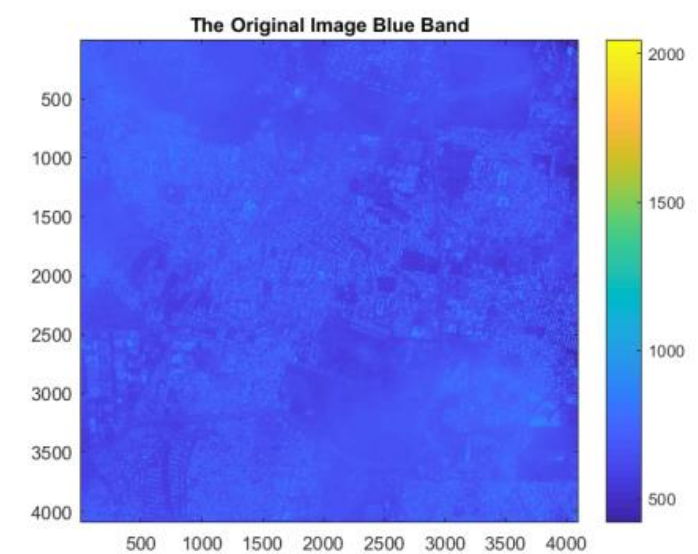

$\begin{array}{llllllll}500 & 1000 & 1500 & 2000 & 2500 & 3000 & 3500 & 4000\end{array}$

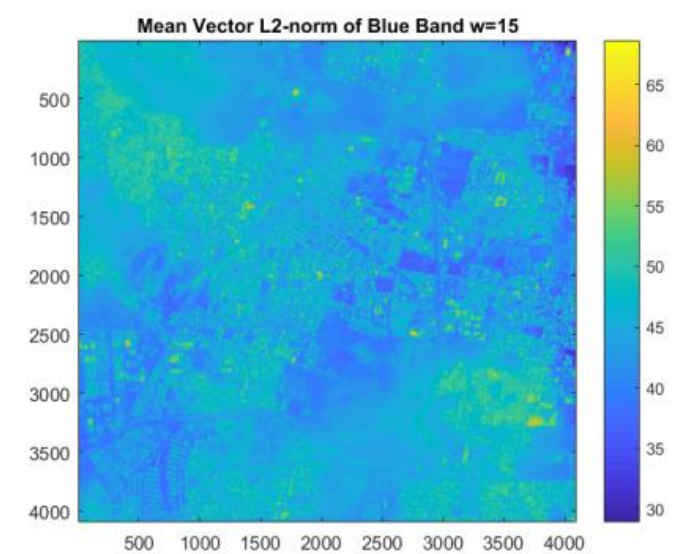

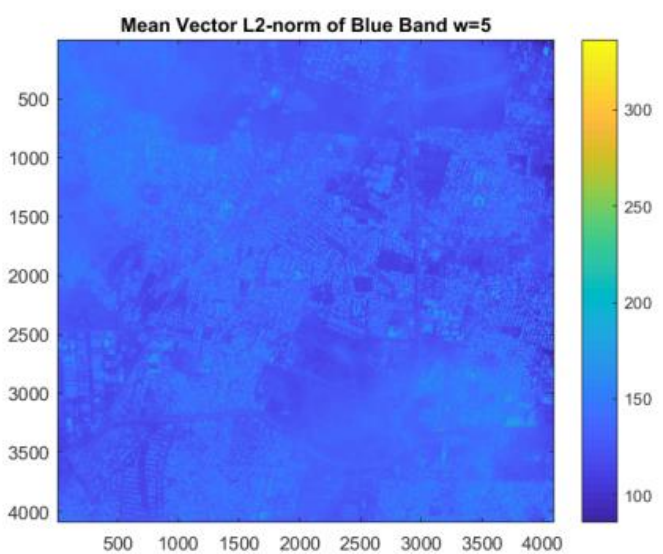

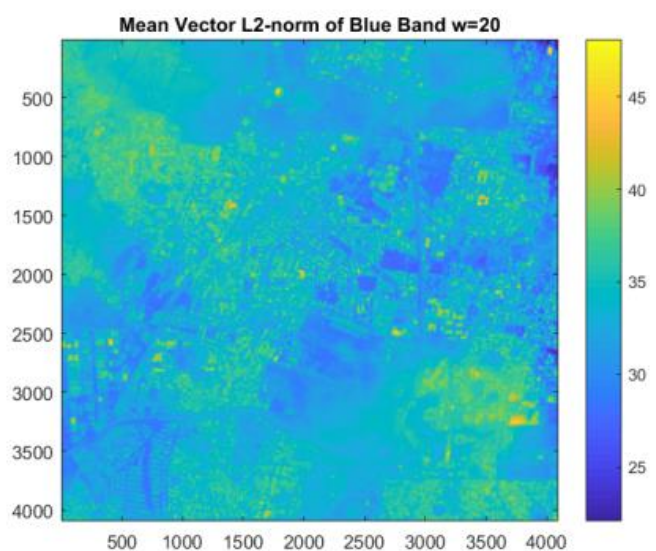

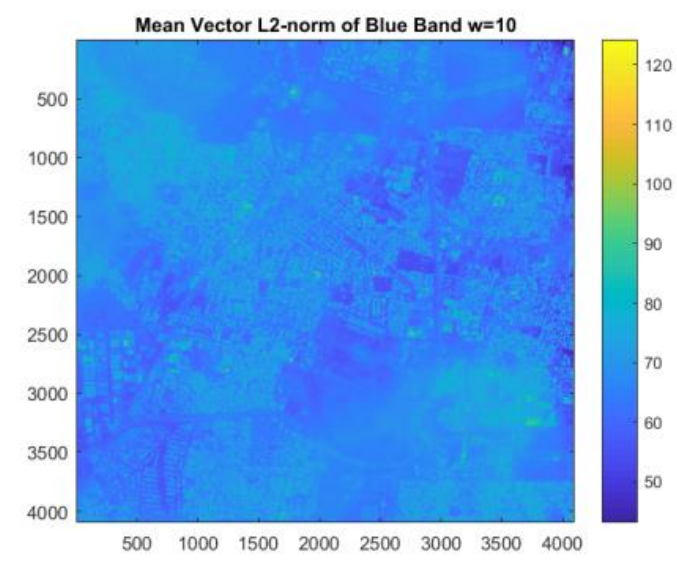

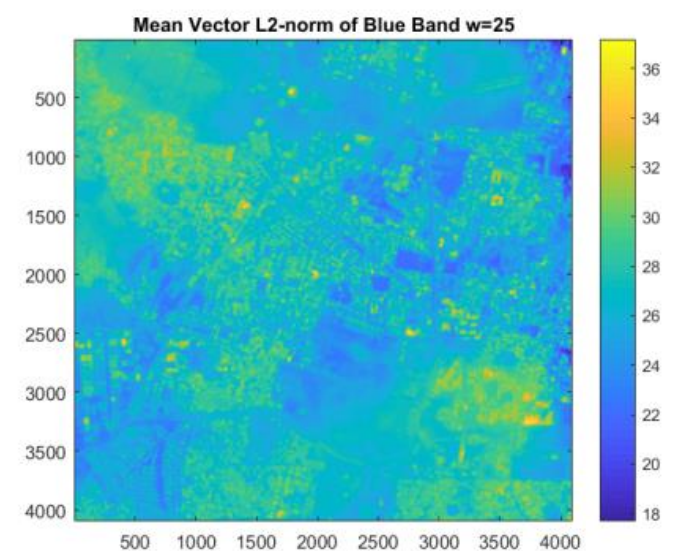

Figure 2. Mean vector $\mathrm{L}^{2}$-norm of the blue band for different window sizes $w$. With the increase of $w$, haze, bright objects and background can be distinguished easily in visual inspection. The location and data source refer to Figure 4. 
The HTM is calculated by searching the darkest pixels using the moving windows in the mean vector $\mathrm{L}^{2}$-norm $\overline{\text { Blue_L } \mathrm{L}^{2}}$ from equation (7) and then resampled to the original spatial resolution.

$$
\operatorname{HTM}(x, y)=\min _{\left(x^{\prime}, y^{\prime}\right) \in W(x, y)}\left(\overline{B l u e_{-} \mathrm{L}^{2}}\left(x^{\prime}, y^{\prime}\right)\right)
$$

where $W(x, y)$ is a moving window, centered at $(x, y), w$ is a bandwidth parameter determining the size of the window, $\overline{B l u e_{-} \mathrm{L}^{2}}(x, y)$ is the mean vector $\mathrm{L}^{2}$-norm of the blue band which is most affected by haze. Larger $w$ helps to eliminate HTM overestimation caused by large bright objects, but also leads to lower edge detection accuracy of the HTM. Smaller $w$ results in more bright ground objects misclassified and included in the HTM.

\subsection{HTM Refinement method}

It is still necessary to label and interpolate bright object pixels for the HTM when the size of those bright objects is larger than $w * w$. Makarau et al. [14] detect bright objects using single threshold segmentation from the red band and interpolate the HTM values in the regions of bright objects. However, the single threshold segmentation cannot distinguish the thick haze regions and the bright objects. We first make a rough distinction between bright and dark pixels according to the given proportion of bright pixels in the whole image from the haze-sensitive band without image segmentation. It is worth noting that the percentage threshold is insensitive to the estimated bright pixels. Thus, for more automated processing requirements, we use $50 \%$ as the default threshold. Secondly, we extract haze and haze-free pixels using the two mean thresholds from bright and dark pixels respectively, in order to mask the haze pixels (see Figure 3. masked HTM). Thirdly, the masked pixels are replaced with the nearest neighbor pixels (see Figure 3. refined HTM). Finally, haze pixels are extracted according to the given percentage (see Figure 3. refined haze map), and a majority filter is used for eliminating large bright objects according to the given minimum size threshold (see Figure 3. refined haze map majority). Compared with the method proposed by Makarau et al. [14], our haze refinement method may theoretically be more robust in dense haze and bright object region and faster because no image segmentation is required. 

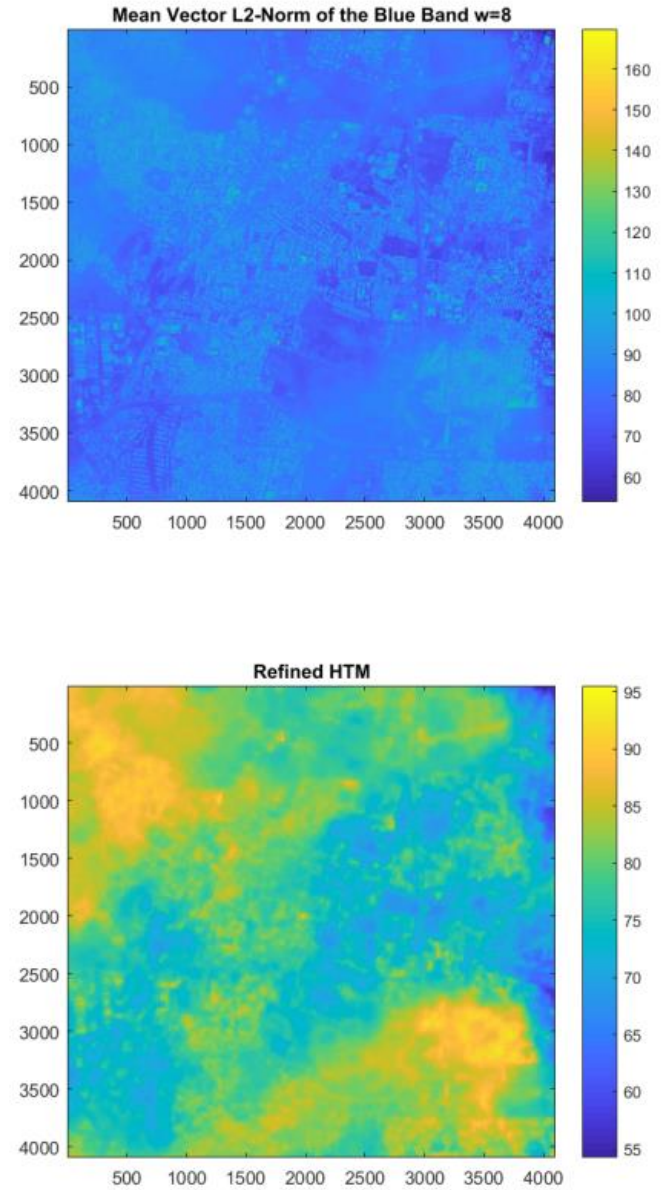

HTM
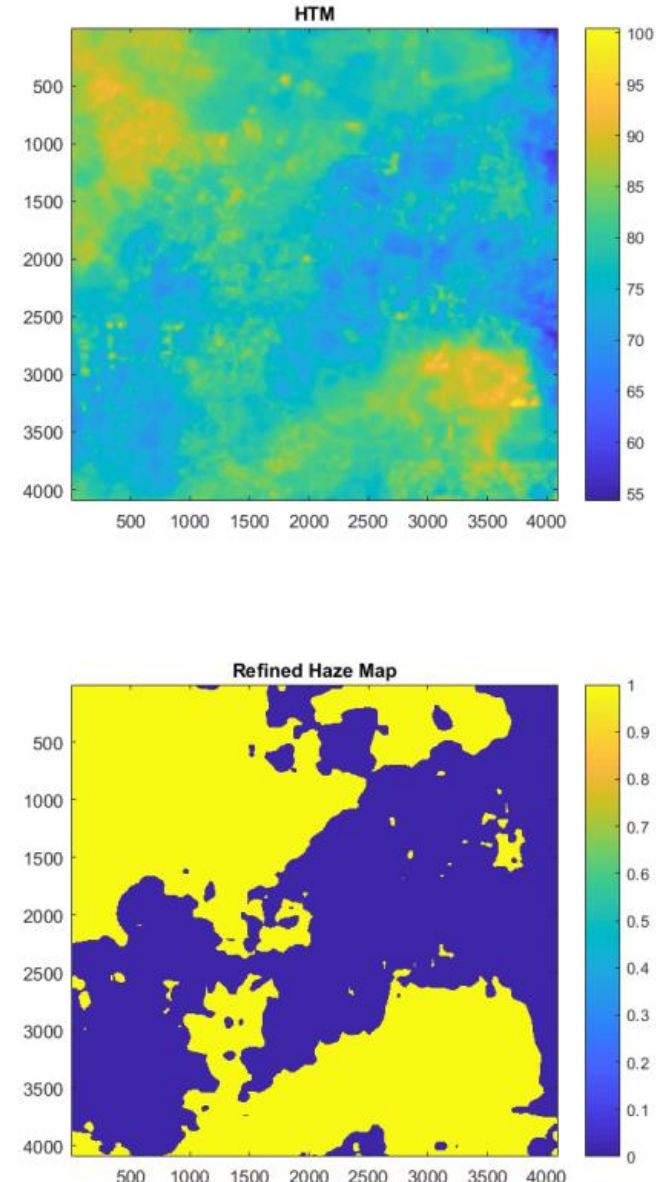
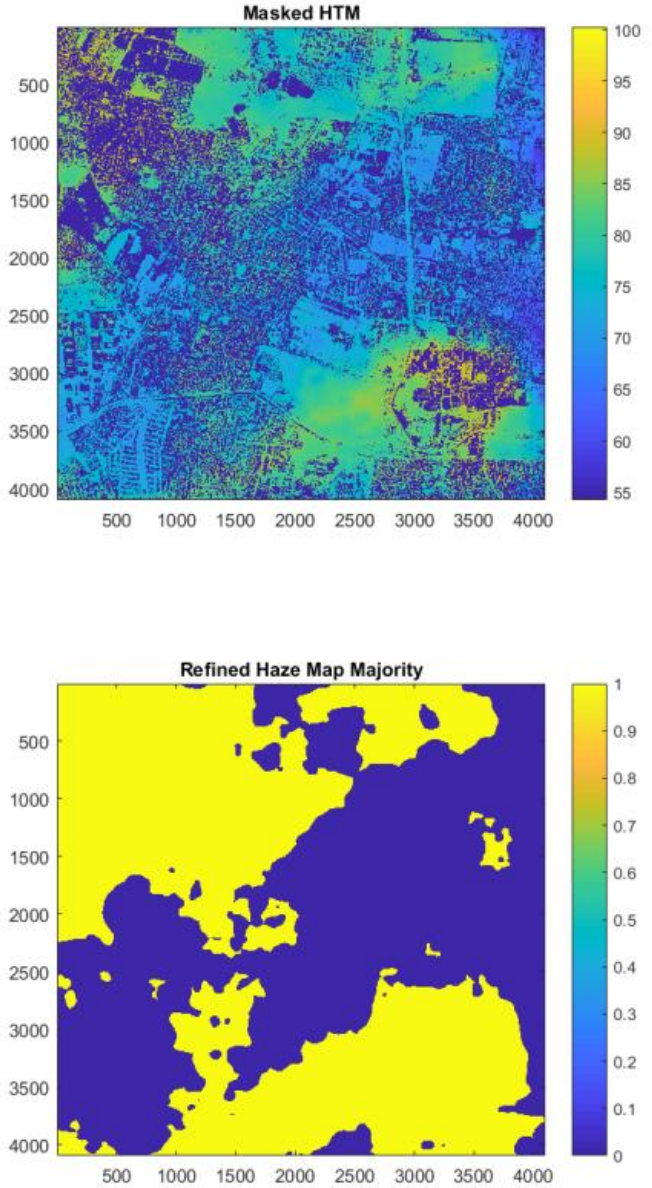

Figure 3. HTM and HTM refinement. The location and data source refer to Figure 4. 


\subsection{HTM calculation for each band}

According to the general haze imaging model described above, the effect of haze in optical satellite images is band-specific and can be subtracted from each band to recover the haze-free image. Longer wavelength radiation can easily penetrate through the haze region and is less affected by haze [14,15]. In general, an accurate HTM is estimated and refined from the most haze-sensitive band; other band-specific $H T M_{i}$ are predicted using linear regression with the dependent $\operatorname{HTM}_{i}(x, y)$ versus the independent $\operatorname{HTM}_{\text {blue }}(x, y)$ (refer equation (2)). All the $H T M_{i}$ can be calculated using a larger window size for accelerating the estimation of the slope and intercept coefficients. Intercept coefficients are close to 0. Compared with the method proposed by Makarau et al. [14,15], slope coefficients are not scaled to [0 1] in the proposed method.

\subsection{De-hazing and compensating}

Along equations (2) and (3), haze-free pixels will be overcorrected, as the subtracted $H_{T} M_{i}$ include haze and haze-free pixels. Thus the compensation should be performed using the mean difference of haze-free pixels between the original and dehazed bands following [14,15]. In practice, it is not easy to detect bright objects in dense haze region of VHR satellite images. Thus, we assume that bright objects will lead to HTM overestimation and dehazed image underestimation in dense haze region. The mean differences between HTM_haze and HTM_hazefree and the relative brightness of the red band are used for compensating the haze pixels as explained in the next section.

\subsection{Proposed method pseudocode}

The proposed method for VHR satellite image de-hazing can be described as pseudocode for better understanding:

${ }^{* *}$ Input: Multispectral data Band $_{i} i=1,2, \ldots, \operatorname{dim} ;$ Percentage threshold $=50 \%$, Minsize $=2500 \mathrm{~m}^{2}$ as the default parameters.

**Output: HTM_refined ; Haze_map; and Band ${ }_{i}^{\text {radiation }}$

${ }^{*}$ Calculate mean vector $\mathrm{L}^{2}$-norm of the blue band using equation (5). Save result as $\overline{B l u e \_L^{2}}$

${ }^{*}$ Calculate HTM using equation (7) and resample to the original size of the image. Save result into HTM.

*Extract Percentage pixels with larger value from HTM as haze pixels $\mathbf{H}$, other pixels as hazefree pixels H_free

*Calculate mean values $\mathbf{H} \_$mean and $\mathbf{H} \_$free_mean

*Mask pixels $\mathbf{H}>\mathbf{H} \_$mean and $\mathbf{H} \_$free $>$H_free_mean from $\mathbf{H T M}$ as HTM_masked 
*Interpolate HTM_masked using nearest neighbor interpolation method save as HTM_refined

*Extract Percentage pixels with larger value as haze pixels $\mathbf{H}$ from $\mathbf{H T M}$ _refined, other pixels as haze-free pixels $\mathbf{H} \_$free

*Obtain binary HTM_map with $\mathbf{H}=1$, and $\mathbf{H} \_$free $=0$

*Filter Haze_map using majority filter with Minsize as Haze_map_majority

*Extract Haze_list and Haze_free_list from Haze_map

${ }^{*}$ Calculate $H T M_{\text {blue }}$ using equation (4)

For $i=1$ to $\operatorname{dim}$

${ }^{*}$ Calculate $H T M_{i}$ using equation (4), using the $i$-th band instead of the blue band

*Estimate slope and intercept coefficients of a linear regression model using ordinary least square fitting

$\left[a_{i}, b_{i}\right]=$ linear regression $\left(H_{T} M_{i}[\right.$ Haze_list $], H T M_{\text {blue }}[$ Haze_list $\left.]\right)$

End

*Perform de-hazing and compensating

For $i=1$ to $\operatorname{dim}$

Band $_{i}^{\text {radiation }}=$ Band $_{i}-a_{i} \times H T M-b_{i}$

$\Delta m e a n_{-}$hazefree $=$mean $\left(\right.$Band $_{i}\left[\right.$ haze $\_$free_list $\left.]\right)-$mean $\left(\right.$Band $_{i}^{\text {radiation }}\left[\right.$ haze $\_$free_list $\left.]\right)$

$\Delta$ meanHTM $=$ mean $($ HTM $[$ haze_list $])-$ mean $(H T M[$ hazefree_list $])$

${ }^{*}$ Compensating all the pixels using the mean difference of radiation from haze-free pixels

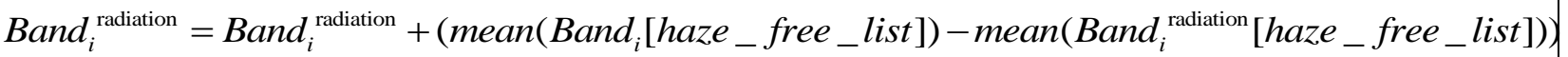

${ }^{*}$ Compensating haze pixels

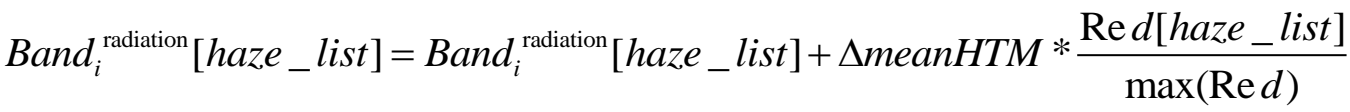

End

\section{Case study and analysis}




\subsection{Study area and dataset}

In order to test the proposed approach, a case study was conducted on one tile of VHR Worldview 3 satellite image captured under severe haze contamination (see Figure 4.). This study was carried out in the very dense built-up region in Bangalore, India covering an area of

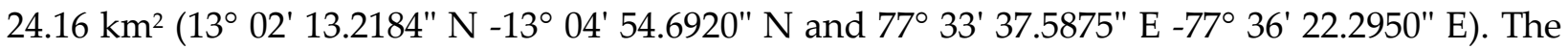
area is covered by a variety of land covers. Due to the spectral similarity between haze and large bright objects in visible bands, the extraction of an accurate HTM is a challenging task. The main parameters of Worldview 3 satellite imagery are as follows: sun elevation: $53.7^{\circ}$, sun azimuth: $159.6^{\circ}$, spatial resolution: $1.2 \mathrm{~m}$, cloud cover: $0 \%$, spatial size: $4096^{*} 4096$. Four bands (NIR-2 - 860nm to $1040 \mathrm{~nm}$, Red $-630 \mathrm{~nm}$ to $690 \mathrm{~nm}$, Green $-510 \mathrm{~nm}$ to $580 \mathrm{~nm}$, Blue $-450 \mathrm{~nm}$ to $510 \mathrm{~nm}$ ) are selected for testing the de-hazing, as these bands are most common in VHR optical satellite images. In this study, the raw digital numbers of Worldview 3 satellite imagery were used for image de-hazing experiments.
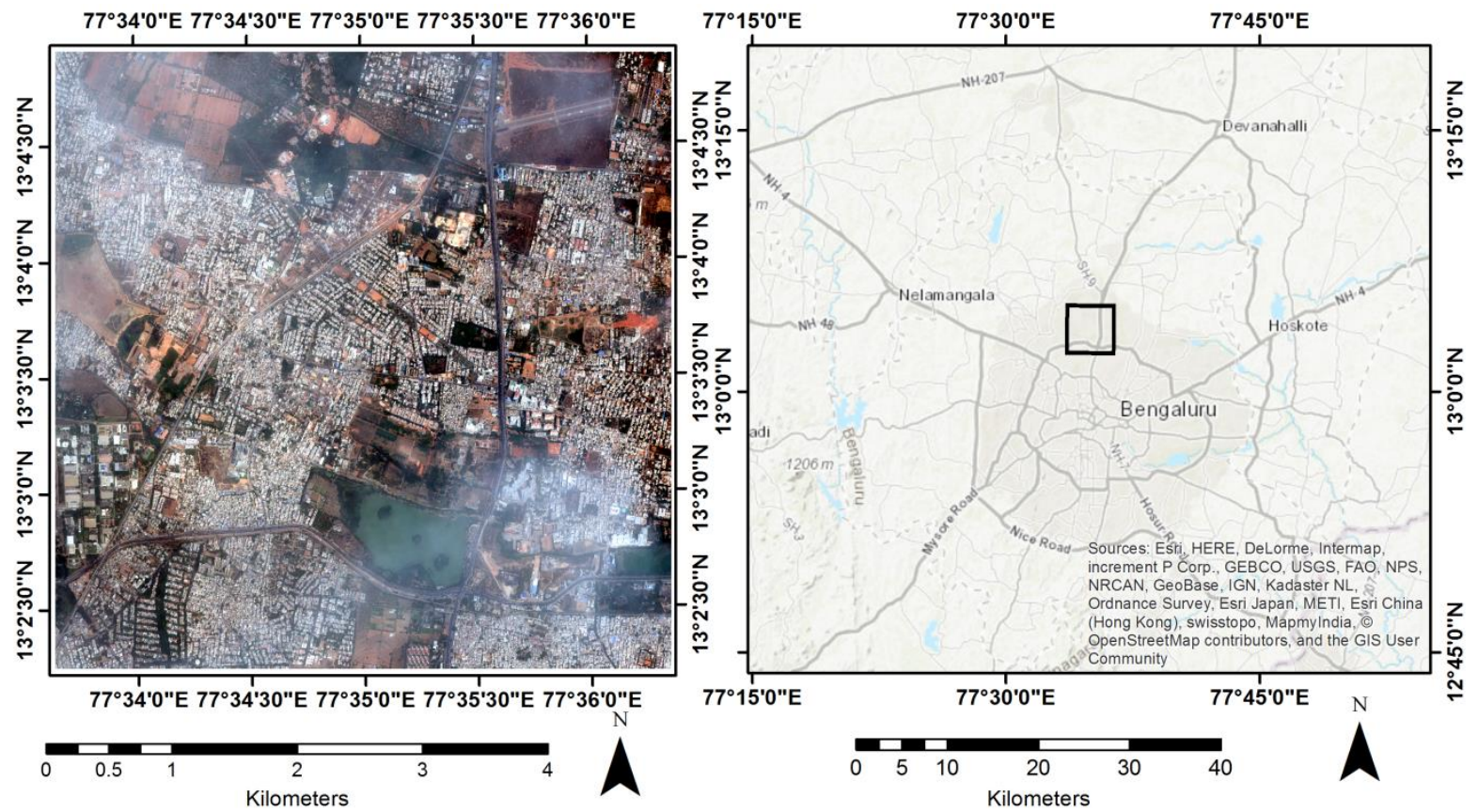

Figure 4. Location of the study site for a case study and Worldview 3 satellite image true color composite (red, green, blue). 
3.2. Visual comparisons
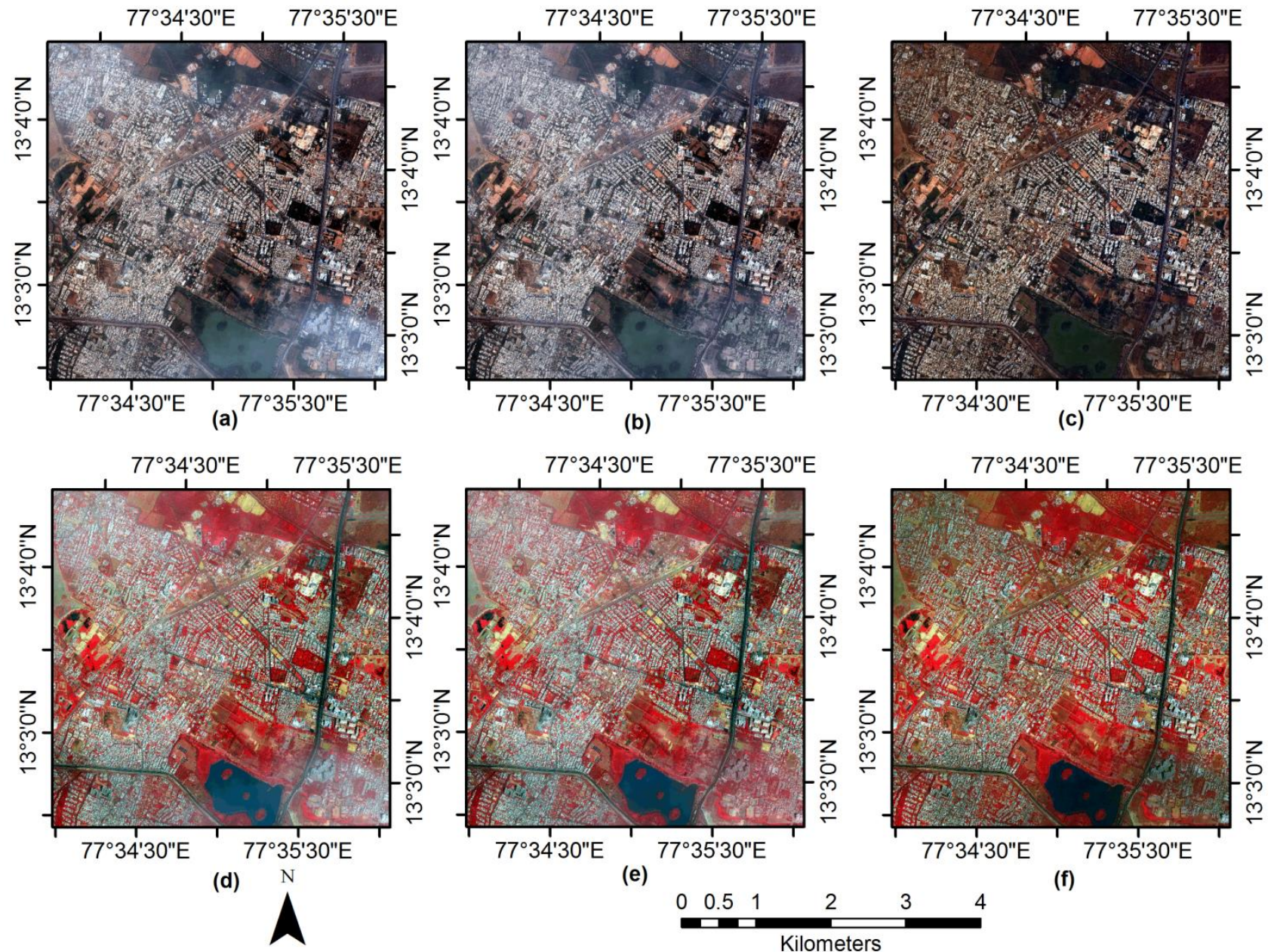

(e)

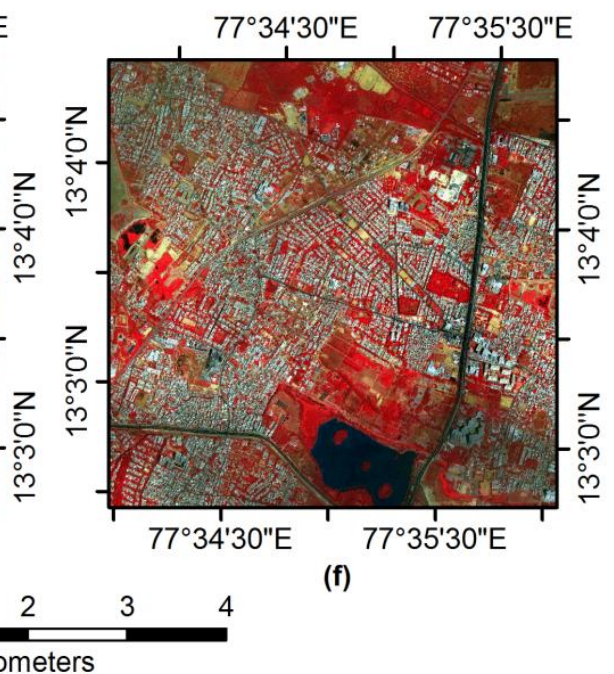

Figure 5. Visual comparisons for Worldview 3 satellite images (subset) (using 2\% linear stretch): the first row shows the true color (red, green, blue) band combination, the second row the corresponding false color (nir-2, red, green) image with: (a) original image, (b) dehazed image by PCI Geomatica 2016, and (c) dehazed image by the proposed method, respectively. Compared with subfigure (b) and (e), subfigure (c) and (f) show that more haze pixels are cleared and restored using the proposed methods. 
It is challenging to collect approximatively synchronous haze and haze-free images with a minimal difference of the sun-sensor geometry from the same VHR satellite sensor. Therefore, evaluation of the de-hazing method was restricted to visual comparison. We applied the cloud detection and haze removal model in PCI Geomatica 2016 as a reference method. The haze removal module of PCI Geomatica is widely accepted and applied by remote sensing scientists. To compare our results with the haze removal method from PCI Geomatica 2016, we set the following parameters: without cloud and water masking and haze cover percentage: $50 \%$ by default.

Figure 5. illustrates the original and dehazed results from the test data. Figure 5. (b) and (e) are the dehazed images produced by PCI Geomatica 2016. Both true color and false color composites show that the haze density is reduced in some areas but not completely removed. Figure 5. (c) and (f) are the corresponding dehazed images produced by the method proposed here, where the calculated slopes were $0.995,1.267,0.831,0.842$ for bands from blue to infrared respectively. The haze was successfully removed while the spectral characteristics of the same land cover from the haze and haze-free areas are consistent as far as visual interpretation allows. Our results show slightly reduced brightness under dense haze areas (e.g. in the region east of the lake), which is because all haze pixels in this region have been detected and removed, but still some large white buildings have been identified as haze pixels, leading to overestimation of the HTM and reduced brightness. As already highlighted above, avoiding HTM overestimation is very important.

\subsection{Quantitative assessment based on image quality metrics}

In general, visual interpretation and comparison can be used to determine whether the image quality after haze removal is better or worse than in the original image. Image quality assessment indicators are used to evaluate whether and to which extent the test images were improved.

\subsubsection{Image quality assessment without reference}

As haze reduces the quality of the image, image quality metrics can be introduced to evaluate the efficiency of image haze removal directly. In the absence of haze-free reference images, no-reference image quality assessment methods are needed for haze remotely sensed imagery. Pan et al. 2016 designed a novel no-reference haze assessment method called haze distribution map based haze assessment (HDMHA) [11]. HDMHA achieved good consistency when compared with visual inspection and outperformed typical blind image quality assessment methods. The intensity of the haze-free region in the HDMHA is close to zero, while the intensity in the haze region is close to one. HDMHA can be used to evaluate image quality for haze and dehazed images. The difference of HDMHA between a haze and dehazed image can be used for assessing the effectiveness of the haze removal method. For each nonoverlapping small window $i$, HDMHA is calculated from equations (8) and (9).

$$
\mathrm{HDMHA}_{i}=\frac{2 \operatorname{mean}_{x_{i} \in \Omega(x)} \mathbf{H}_{\mathbf{s}}(\mathbf{y})}{\max \left(T, \max _{x_{i} \in \Omega(x)} \mathbf{H}_{\mathbf{s}}(\mathbf{y})\right)+\min _{x_{i} \in \Omega(x)} \mathbf{H}_{\mathbf{s}}(\mathbf{y})}
$$


where $H_{s}(y)$ is the haze distribution map produced from true color band combination considering the saturation of the image, $\Omega(x)$ is a local patch centered at $x$ with window size $=20$, more detailed information we refer to Pan et al. 2016 [11]. $T$ is set to 0.8 and a patch is composed of $20^{*} 20$ pixels [11].

For a whole image, HDMHA metric is expressed as

$$
\text { HDMHA }=\frac{1}{n} \sum_{i=1}^{n} \text { HDMHA }_{i}
$$

Where $n$ is the number of the patches, according to (8) and our experiments, HDMHA $_{i}$ cannot distinguish haze or large bright object directly from VHR visual satellite images; it means that $\mathrm{HDMHA}_{i}$ of big bright objects have high values, similar to haze regions. A high HDMHA value indicates dense and widespread haze in a remotely sensed image. Thus, a lower HDMHA value indicates better quality of the image or a better performance of image dehazing.

\subsubsection{Image quality assessment with reference}

Mean-squared Error (MSE), Peak Signal-to-noise Ratio (PSNR) [20], and Structural Similarity Index (SSIM) [21] are three most well-known image quality metrics. MSE measures the mean squared difference between the actual and ideal pixel values, which is easy to calculate. PSNR is derived from the mean square error, which represents the ratio of the maximum pixel intensity to the power of the distortion. SSIM combines the local image structure, brightness, and contrast into an individual local quality value. In this metric, the structure is the pattern of pixel intensities, especially among adjacent pixels, and the brightness and contrast are normalized. Because the human visual system is good at perceiving structure, the SSIM is closer to a subjective image quality evaluation. For more details on these three metrics, please refer to the cited literature.

Normally, these image quality metrics need high-quality images as references. Here, we use the original RGB haze image (low-quality image) as the reference, which is an accepted approach as mentioned in literature [22]. In cases where all the test images are of higher quality (judged image quality by visual interpretation) than the original haze-contaminated image, higher values of MSE, lower values of PSNR and lower values of SSIM indicate better performance of image haze removal. It is necessary to emphasize that all of these values are computed in the RGB channels.

\subsubsection{Image quality assessment results}

Table 1 shows the quantitative evaluation results using different evaluation criteria. HDMHA metrics point out that the haze removal model of PCI Geomatica 2016 does not clearly improve the quality of the haze image. The HDMHA value of the proposed method is not close to 0 . It is because of the limitation of HDMHA which cannot well distinguish bright objects and

haze pixels in VHR satellite images. Although HDMHA has such drawbacks, it also can 
evaluate the comparative advantage of different de-hazing methods. Thus, lower values may be taken as an indication that the proposed method offers a significant quality enhancement according to the HDMHA indicator. Given a haze image is the reference, lower values of PSNR and SSIM means that the proposed method shows a better result. Larger MSE value from the proposed method means that difference between the dehazed image and haze image are larger. A larger MSE together with the visual comparison can confirm that the proposed method outperforms the method implemented in PCI Geomatica 2016. All four indicators show better performance of the proposed method in this example.

Table 1 Quantitative assessment of different image quality measures

\begin{tabular}{lllll}
\hline RGB Image & HDMHA & PSNR & SSIM & MSE \\
\hline Original & 0.4998 & - & 1 & 0 \\
PCI Geomatica 2016 & 0.5004 & 25.2620 & 0.9749 & 193.5868 \\
Proposed & 0.2590 & 15.3763 & 0.7533 & 1885.6000 \\
\hline
\end{tabular}

\subsection{Comparison and analysis of spectral characteristics}

Changes in the spectral characteristics changes of objects before and after haze removal are also of interest, because they directly affect the subsequent image processing and applications, such as the calculation of vegetation indices, land cover classification and others. Figure 6 illustrates the dehazed results for a Worldview 3 subscene. Visual interpretation and comparison of the dehazed image from the proposed method (Figure 6 (c)) and from PCI Geomatica 2016 (Figure 6(b)) help to confirm the conclusion that the proposed method performs better.

Further, comparisons of the channel intensity (DN) profiles in the haze and dehazed images are illustrated in Figure 7. In haze-free regions, the spectral profiles in the original and dehazed image have similar curves. In haze regions, the dehazed profiles show more noticeable dehazing enhancement of the intensities from the red, green, and blue bands when the proposed method is used. For the same bare land in Figure 6(c) and Figure 7(c), the spectra collected from the haze region have the more consistent shape as the spectra collected in the haze free region. Thus, the proposed method produces spectrally more consistent dehazed images. 

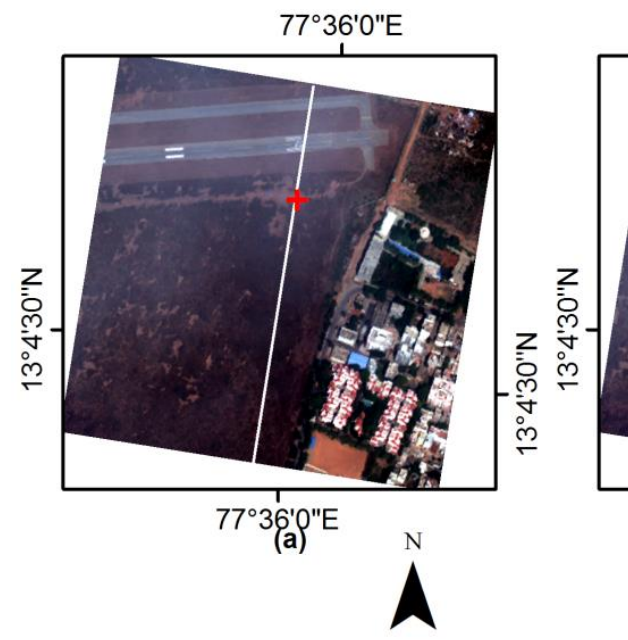

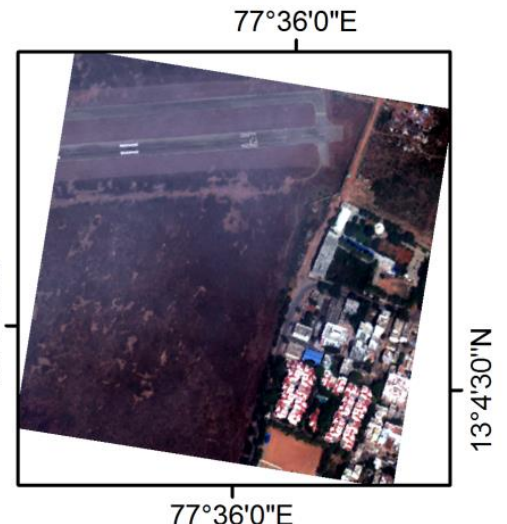

(b)

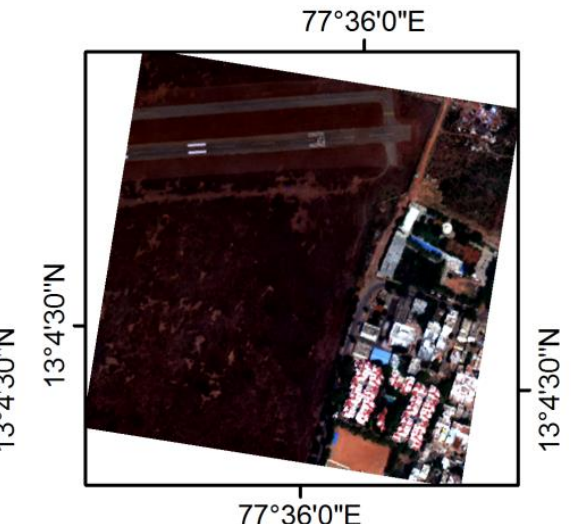

0.6

Kilometers

Figure 6. Dehazing of Worldview 3 data (subset). (a) Haze image RGB true color band combination, (b) dehazed image RGB band combination by PCI Geomatica 2016 and (c) dehazed image RGB band combination with the proposed method.

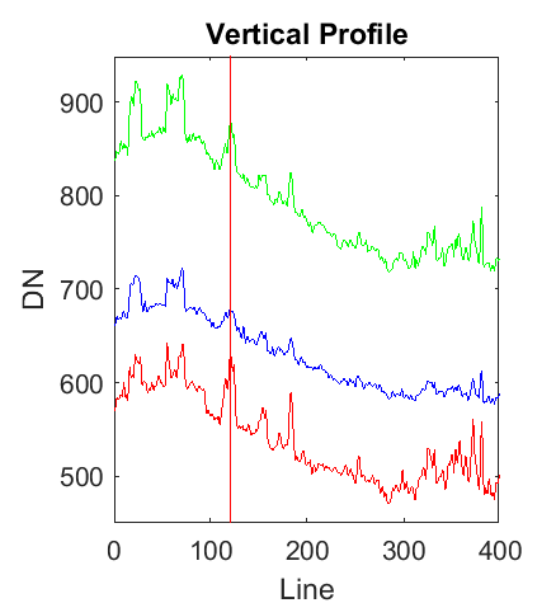

(a)

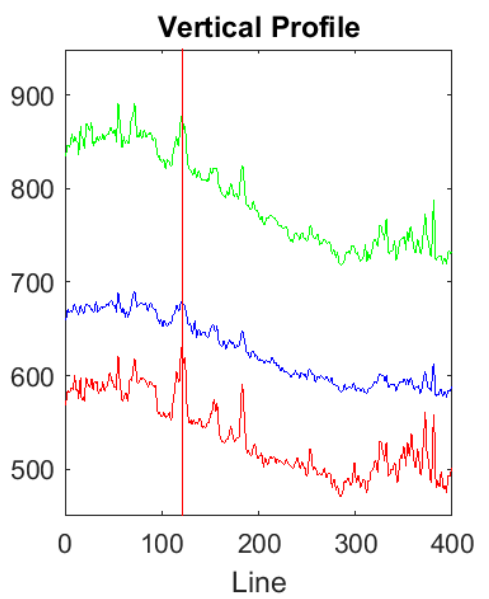

(b)

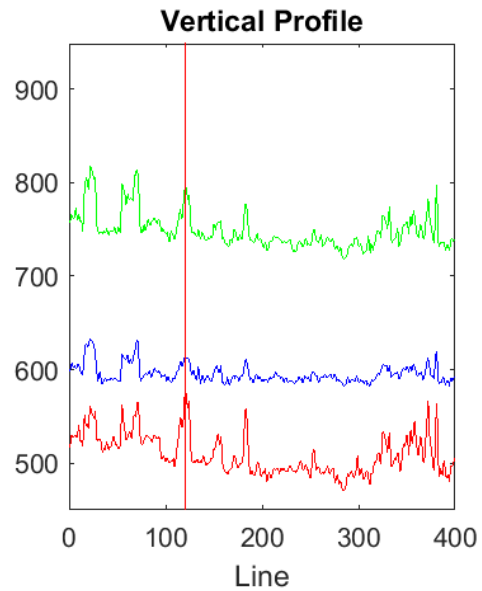

(c)

Figure 7. Spectral profiles (Figure 6 (a), white line ) taken from (a) the original haze image, (b) dehazed image by PCI Geomatica 2016, and (c) dehazed image by the proposed method. The red, green, and blue spectral profiles represent the DN values of the red, green and blue band respectively. The spectral values of the vertical red solid line come from the pixel of the marked location (plus symbol) in Figure 6 (a). Pixels of this location show mainly bare land with high homogeneity, so their real spectral curves should be relatively horizontal. Differences between (a) and (c) illustrate more clearly that the performance of the proposed method is more noticeable while the shape of the profiles after haze removal indicate maintenance of the scene structure in the red, green and blue bands.

Below, we analyzed the changes of the vegetation pixel samples before and after the haze removal. Vegetation pixels are extracted from 4 rectangular regions ( 2 haze free regions and 2 haze affected areas), which consist of a total of 7065 vegetation pixels, including 3571 haze free pixels and 3494 haze pixels. We analyzed the intra-class variability of vegetation pixels using pairs of boxplots, as boxplot has obvious advantages in representing datasets and statistical parameters [23] using the 25th, 50th, and 75th percentiles. Figure 8 shows the difference in 
distributions of the DN values of vegetation pixel samples between the original image and the two dehazed images for RGB bands. From the proposed method, the interquartile ranges of the RGB bands from the dehazed image are the narrowest compared to those from the original image and dehazed image resulting from PCI Geomatica. These findings indicate that the red, green, and blue bands become more homogeneous after haze removal with the proposed method.

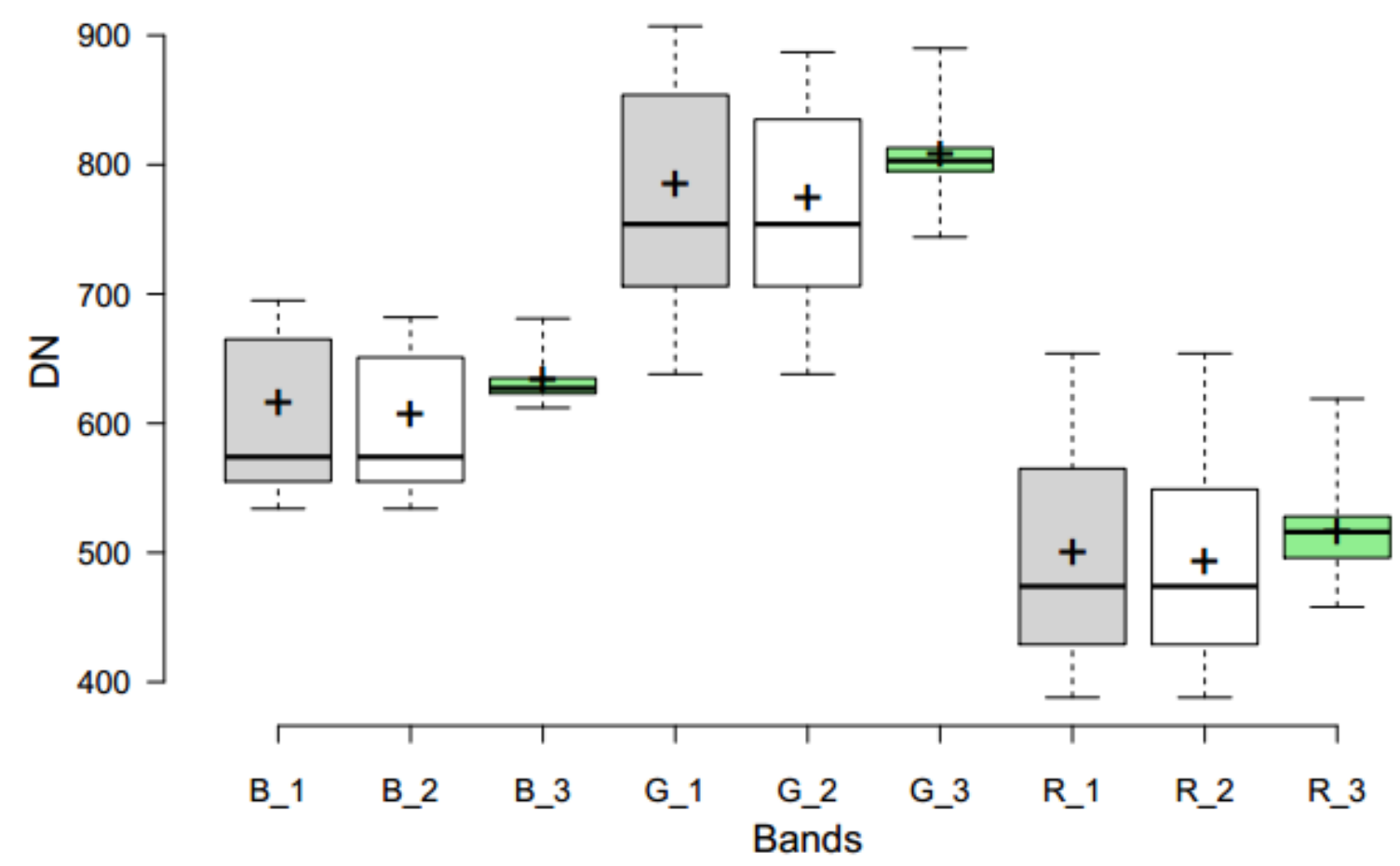

Figure 8. Boxplot comparisons of the DN values of vegetation pixel samples for the original and dehazed images from PCI Geomatica 2016 and the proposed methods across red, green, and blue bands. RGB bands (R - 630nm to 690nm, G - 510nm to 580nm, B - 450nm to 510nm) of Worldview 3 are used. "_1", “_2", and “_3” represent the original image (light gray), dehazed image from PCI Geomatica 2016 (white), and dehazed image from the proposed method (light green), respectively. Boxplot description for this figure legend: Center lines show the medians; box limits indicate the 25 th and 75 th percentiles as determined by $\mathrm{R}$ software; whiskers extend to minimum and maximum values; crosses represent sample means. $n=7065$ sample points.

\subsection{Computational complexity}

The computer codes were developed in the Matlab 2014b platform, running on a PC with Intel(R) Core(TM) i7-3520M CPU @ 2.90 GHz, 8G RAM, and 64-bit Windows 7 Professional Operating System. The computational complexity was analyzed in the four major tasks. It takes a total of 47.3 seconds to de-haze the Worldview 3 satellite image (4 bands and $4096 * 4096$ pixels). Calculation of mean vector $\mathrm{L}^{2}$-norm of the blue band takes 20.3 seconds, HTM refinement takes 15.1 seconds, band-specific HTM calculation takes 3.6 seconds, and image dehazing together with compensating takes 5.3 seconds. Experience shows that the first two tasks are most time-consuming, their computing time increases linearly with the number of pixels in the haze-sensitive band and exponentially with the given window size. Meanwhile, the latter two tasks are very fast while their execution time is related to the number of bands. Image de- 
hazing and compensating tasks will still be effective when applied to images with more bands. The haze removal method requires nearly 2 minutes for the de-hazing 8-bands image using the haze removal model from the atmospheric correction module implemented in PCI Geomatica 2016. It is worth noting that processing times of less than one minute were achieved when the proposed method was applied to 8-band images of the same extent (4096x4096 pixels). The computational efficiency can be realized because the proposed method uses the nearest neighbor interpolation and does not need prior image segmentation in the process of the HTM refinement.

\section{Haze removal performance in a wide variety of data sources}

In the previous section, visual comparisons, quantitative evaluation methods with- and without reference images, spectral characteristics analysis and computational efficiency were described in detail and used to evaluate the performance of the proposed method in haze removal using a case study in Bangalore and Worldview 3 data. These methods can repeatedly be used to evaluate the performance of the proposed method in any new VHR optical satellite image. Here, more applications of the proposed method are simply shown in a variety of data sources with different spatial resolution. Meanwhile, because visual interpretation, presentation, and printing are important areas of application of remote sensing images, we only visually demonstrate comparisons of their performance before and after haze removal using the proposed method. Detailed information on the test images is given in Table 2. The visual comparisons show that the proposed method achieved good haze removal performance in various VHR satellite data (see supplementary materials, Figure S1 - Figure S4). Further, HDMHA metrics of dehazed images are significantly lower than those of the original images (see Table 3), when the proposed method is used in these various spatial resolution VHR satellite data.

Table 2 Parameters for VHR satellite images for testing.

\begin{tabular}{lllll}
\hline Data source & Worldview3 & Worldview 3 & Pléiades 1-B & Gaofen-2 \\
\hline Place & $\begin{array}{l}\text { Shanghai, } \\
\text { China }\end{array}$ & New Delhi, India & Chenxi, China & $\begin{array}{l}\text { Yuanjiang, } \\
\text { China }\end{array}$ \\
Acquired date & April 18, 2016 & September 28, 2017 & August 22, 2014 & May 7, 2015 \\
Solar elevation & $65.80^{\circ}$ & $58.30^{\circ}$ & $67.05^{\circ}$ & $70.54^{\circ}$ \\
Solar azimuth & $142.30^{\circ}$ & $163.00^{\circ}$ & $130.74^{\circ}$ & $125.04^{\circ}$ \\
GSD & $1.20 \mathrm{~m}$ & $1.20 \mathrm{~m}$ & $2.00 \mathrm{~m}$ & $3.24 \mathrm{~m}$ \\
\hline
\end{tabular}

Table 3 HDMHA performance of different VHR satellite images before and after haze removal using the proposed method

\begin{tabular}{lllll}
\hline RGB Image & Worldview 3 of Shanghai & Worldview 3 of New Delhi & Pléiades 1-B & Gaofen-2 \\
\hline Original & 0.4998 & 0.5910 & 0.3180 & 0.4586 \\
Proposed & 0.2590 & 0.3746 & 0.2494 & 0.2720 \\
\hline
\end{tabular}




\section{DISCUSSION}

It is well known that haze removal is an important pre-processing step for further image processing and applications of optical satellite images. Although various haze removal methods have been proposed in recent years, the evaluation of the performance of a new proposed method still faces challenges.

In this study, we used the haze removal model of PCI Geomatica 2016 as a reference method because of its proprietary technologies, high acceptance and usage. We have tested all the data mentioned in this study with Geomatica 2016. Haze pixels are not significantly removed (not all data is shown), especially in urban and densely built-up areas. The parameter free strategy may be the reason for the relatively poor performance in this study and it may be not necessarily well adapted to VHR satellite images of urban areas with intensive bright objects. Thus, it is worth emphasizing that we cannot conclude that the proposed method has absolute advantages over other methods and we just provide another option for haze removal technology in VHR optical satellite imagery.

Although some evaluation methods have been proposed, including reference- [24], without reference- [11] and training image database [25-27] based haze removal evaluation metrics, there is still a lack of universally accepted indicators to evaluate haze removal performance, particularly for VHR optical satellite imagery.

As shown in the visual comparisons, slightly reduced brightness appears in some hazedense areas. It is because haze pixels in the haze-dense area are detected and removed, but the compensation for haze pixels of the area is incomplete. The haze removal task needs to determine the trade-off between the removal rate of haze and the fidelity of spectral features, especially for optical satellite images. The proposed method takes the elimination of haze as the

primary task and the recovery of spectral properties as a subsequent task. Meanwhile, it is worth noting that haze removal does not mean complete spectral recovery, which may be also unlikely to be fully realized in practice.

\section{CONCLUSIONS}

In this paper, we presented a robust and straightforward method for VHR optical satellite image de-hazing. Very dark pixels in the haze region cause underestimation in the HTM, which can be prone to partially missed haze inspection and haze residue. In addition, confusion of bright ground objects and haze lead to an overestimation in the HTM, which can further lead to under-de-hazing of the image. For these reasons, we proposed using a mean vector $\mathrm{L}^{2}$-norm of the haze-sensitive band and two thresholds based bright objects removal strategy to avoid under- or over- estimation of HTM. As the effects of bright objects cannot be excluded completely in practice, it still leads to HTM overestimation and further to over-de-hazing. Pixels in haze regions are compensated using the mean difference of HTM between haze and haze-free pixels. The compensation is assigned to each pixel in haze region according to the relative brightness of the red band. 
Experiments on several VHR optical satellite images in different places, including Worldview 3, Pléiades and Gaofen-2 images, validate the effectiveness of the proposed method. The performance of the method is initially compared with the PCI Geomatica 2016 based haze removal method in detail both visually and quantitatively for one Worldview 3 image. From the results of the experiment, it is clear that the proposed image de-hazing method can remove haze from VHR satellite images without significantly reducing effects on its natural appearance. Image quality metrics (with or without reference) indicate that the proposed approach shows good performance. Meanwhile, the proposed method is less computationally intensive.

We have tested a variety of VHR optical satellite data with the spatial resolution less than 5 meters, and the results were satisfying. The advantages of this technique are that aerosol transparent bands are not needed, the technique can be applied to original data DN values or calibrated radiance data, and the technique is suitable for the general VHR optical satellite images.

Cloud detection and cloud masking are not included in this study, but they can be integrated into the proposed method as part of the image pre-processing. Due to the lack of real reference data, the subsequent applications of dehazed images such as image classification, change detection, etc. can be used to evaluate the performance of different methods of haze removal indirectly. Moreover, the spectral consistency analysis without reference should be studied in the future.

\section{Supplementary Materials}

The following are available online at www.mdpi.com. Figure S1. Haze removal performance on Worldview3 satellite imagery (Shanghai, China, GSD = $1.2 \mathrm{~m}$ ) (using 2\% linear stretch). Figure S2. Haze removal performance on Worldview3 satellite imagery (New Delhi, India, GSD = $1.2 \mathrm{~m}$ ) (using 2\% linear stretch). Figure S3. Haze removal performance on Pléiades satellite imagery (Chenxi, China, GSD = $2 \mathrm{~m}$ ) (using 2\% linear stretch). Figure S4. Haze removal performance on Gaofen-2 satellite imagery (Yuanjiang, China, GSD = $3.24 \mathrm{~m}$ ) (using 2\% linear stretch).

Acknowledgements: This research is an outcome of various research projects in which the authors were involved, and the authors do sincerely thank the following funding institutions: the German Federal Ministry of Education (BMBF) (Project Lin4Carbon, Grant No. 033L049C), Deutsche Forschungsgemeinschaft (DFG) (Research Group FOR2432), the Chinese Academy of Forestry (CAF) (Grant No. Lin2Value-CAFYBB2012013), and the National Natural Science Foundation of China (NSFC) (Grant No. 31470643 and 31100412). We also thank the supplier of Worldview 3 data: WorldView Imagery (C) 2017 DigitalGlobe, Inc. - provided by European Space Imaging. The authors would like to thank Dr. Haijun Yang for technical suggestions.

Author Contributions: Dengkui Mo, Hans Fuchs, and Lutz Fehrmann designed the study. Nils Nölke collected Worldview 3 satellite sample images. Dengkui Mo designed and developed the algorithm in the manuscript. Dengkui Mo, Hans Fuchs, and Lutz Fehrmann completed the calculations, map production, and the image quality assessment. Dengkui Mo completed the first version of the draft. Lutz Fehrmann, Hans Fuchs, Nils Nölke, Paul Magdon, and Christoph Kleinn revised the manuscript.

Conflicts of Interest: The authors declare no conflict of interest. 


\section{References}

1. Liu, S.; Rahman, M.A.; Wong, C.Y.; Lin, C.-F.; Wu, H.; Kwok, N. Image de-hazing from the perspective of noise filtering. Comput. Electr. Eng. 2016.

2. Fattal, R. Single image dehazing. ACM transactions on graphics (TOG) 2008, 27, 72.

3. Tan, R.T. In Visibility in bad weather from a single image, Computer Vision and Pattern Recognition, 2008. CVPR 2008. IEEE Conference on, 2008; IEEE: pp 1-8.

4. He, K.; Sun, J.; Tang, X. Single image haze removal using dark channel prior. IEEE. T. Pattern. Anal. 2011, 33, 2341-2353.

5. Xiao, C.; Gan, J. Fast image dehazing using guided joint bilateral filter. The Visual Computer 2012, 28, 713-721.

6. He, K.; Sun, J.; Tang, X. Guided image filtering. IEEE transactions on pattern analysis and machine intelligence 2013, 35, 1397-1409.

7. Zhu, Q.; Mai, J.; Shao, L. A fast single image haze removal algorithm using color attenuation prior. IEEE. T. Image Process 2015, 24, 3522-3533.

8. Xu, H.; Guo, J.; Liu, Q.; Ye, L. In Fast image dehazing using improved dark channel prior, Information Science and Technology (ICIST), 2012 International Conference on, 2012; IEEE: pp 663-667.

9. Chavez, P.S. An improved dark-object subtraction technique for atmospheric scattering correction of multispectral data. Remote Sens. Environ. 1988, 24, 459-479.

10. Long, J.; Shi, Z.; Tang, W.; Zhang, C. Single remote sensing image dehazing. IEEE Geosci. Remote Sens. Lett. 2014, 11, 59-63.

11. Pan, X.; Xie, F.; Jiang, Z.; Shi, Z.; Luo, X. No-reference assessment on haze for remote-sensing images. IEEE Geosci. Remote Sens. Lett. 2016, 13, 1855-1859.

12. Pan, X.; Xie, F.; Jiang, Z.; Yin, J. Haze removal for a single remote sensing image based on deformed haze imaging model. IEEE Signal Processing Letters 2015, 22, 1806-1810.

13. Zhang, Y.; Guindon, B.; Cihlar, J. An image transform to characterize and compensate for spatial variations in thin cloud contamination of landsat images. Remote Sens. Environ. 2002, 82, 173187.

14. Makarau, A.; Richter, R.; Müller, R.; Reinartz, P. Haze detection and removal in remotely sensed multispectral imagery. IEEE. T. Geosci. Remote 2014, 52, 5895-5905.

15. Makarau, A.; Richter, R.; Schläpfer, D.; Reinartz, P. Combined haze and cirrus removal for multispectral imagery. IEEE Geosci. Remote Sens. Lett. 2016, 13, 379-383.

16. Richter, R. Correction of satellite imagery over mountainous terrain. Appl. Opt. 1998, 37, 40044015.

17. Richter, R. Correction of atmospheric and topographic effects for high spatial resolution satellite imagery. Int. J. Remote Sens. 1997, 18, 1099-1111.

18. Richter, R. Atmospheric correction of satellite data with haze removal including a haze/clear transition region. Comput. Geosci-uk. 1996, 22, 675-681.

19. Jha, D.K.; Gupta, B.; Lamba, S.S. L 2-norm-based prior for haze-removal from single image. IET Comput. Vision 2016, 10, 331-341.

20. Huynh-Thu, Q.; Ghanbari, M. Scope of validity of psnr in image/video quality assessment. Electron. Lett 2008, 44, 800-801.

21. Zhou, W.; Bovik, A.C.; Sheikh, H.R.; Simoncelli, E.P. Image quality assessment: From error visibility to structural similarity. IEEE. T. Image Process 2004, 13, 600-612. 
22. Jiang, H.; Lu, N.; Yao, L. A high-fidelity haze removal method based on hot for visible remote sensing images. Remote Sensing 2016, 8, 844.

23. Spitzer, M.; Wildenhain, J.; Rappsilber, J.; Tyers, M. Boxplotr: A web tool for generation of box plots. Nat. Methods 2014, 11, 121-122.

24. Wang, Z.; Bovik, A.C.; Sheikh, H.R.; Simoncelli, E.P. Image quality assessment: From error visibility to structural similarity. IEEE. T. Image Process 2004, 13, 600-612.

25. Mittal, A.; Moorthy, A.K.; Bovik, A.C. No-reference image quality assessment in the spatial domain. IEEE. T. Image Process 2012, 21, 4695-4708.

26. Mittal, A.; Moorthy, A.; Bovik, A. In Referenceless image spatial quality evaluation engine, 45th Asilomar Conference on Signals, Systems and Computers, 2011.

27. Mittal, A.; Soundararajan, R.; Bovik, A.C. Making a "completely blind" image quality analyzer. IEEE. Signal Proc. Let 2013, 20, 209-212.

(C) 2017 by the authors. Submitted for possible open access publication under the 


\title{
A Simple and Robust Haze Removal Method for VHR Optical Satellite Image Based on Mean Vector L'2-norm
}

\author{
Dengkui Mo ${ }^{1,2,3 *}$, Hans Fuchs' ${ }^{1}$ Lutz Fehrmann ${ }^{1}$, Nils Nölke ${ }^{1}$, Paul Magdon ${ }^{1}$, Christoph Kleinnn,* \\ 1 Forest Inventory and Remote Sensing, Faculty of Forest Sciences and Forest Ecology, Georg-August-Universität \\ Göttingen, Büsgenweg 5, 37077 Göttingen, Germany \\ 2 Research Center of Forestry Remote Sensing \& Information Engineering, Central South University of Forestry \\ and Technology, Changsha 410004, China \\ 3 Key Laboratory of Forestry Remote Sensing Based Big Data \& Ecological Security for Hunan Province, Changsha \\ 410004, China \\ * Correspondence: dmo@gwdg.de; CKleinn@gwdg.de; Tel.: +49 [0]551 39-33472
}

Figure legends

Figure S1. Haze removal performance on Worldview3 satellite imagery (Shanghai, China, GSD = $1.2 \mathrm{~m}$ ) (using 2\% linear stretch).

Figure S2. Haze removal performance on Worldview3 satellite imagery (New Delhi, India, GSD = $1.2 \mathrm{~m}$ ) (using 2\% linear stretch).

Figure S3. Haze removal performance on Pléiades satellite imagery (Chenxi, China, GSD $=2 \mathrm{~m}$ ) (using $2 \%$ linear stretch).

Figure S4. Haze removal performance on Gaofen-2 satellite imagery (Yuanjiang, China, GSD $=3.24 \mathrm{~m}$ ) (using $2 \%$ linear stretch). 

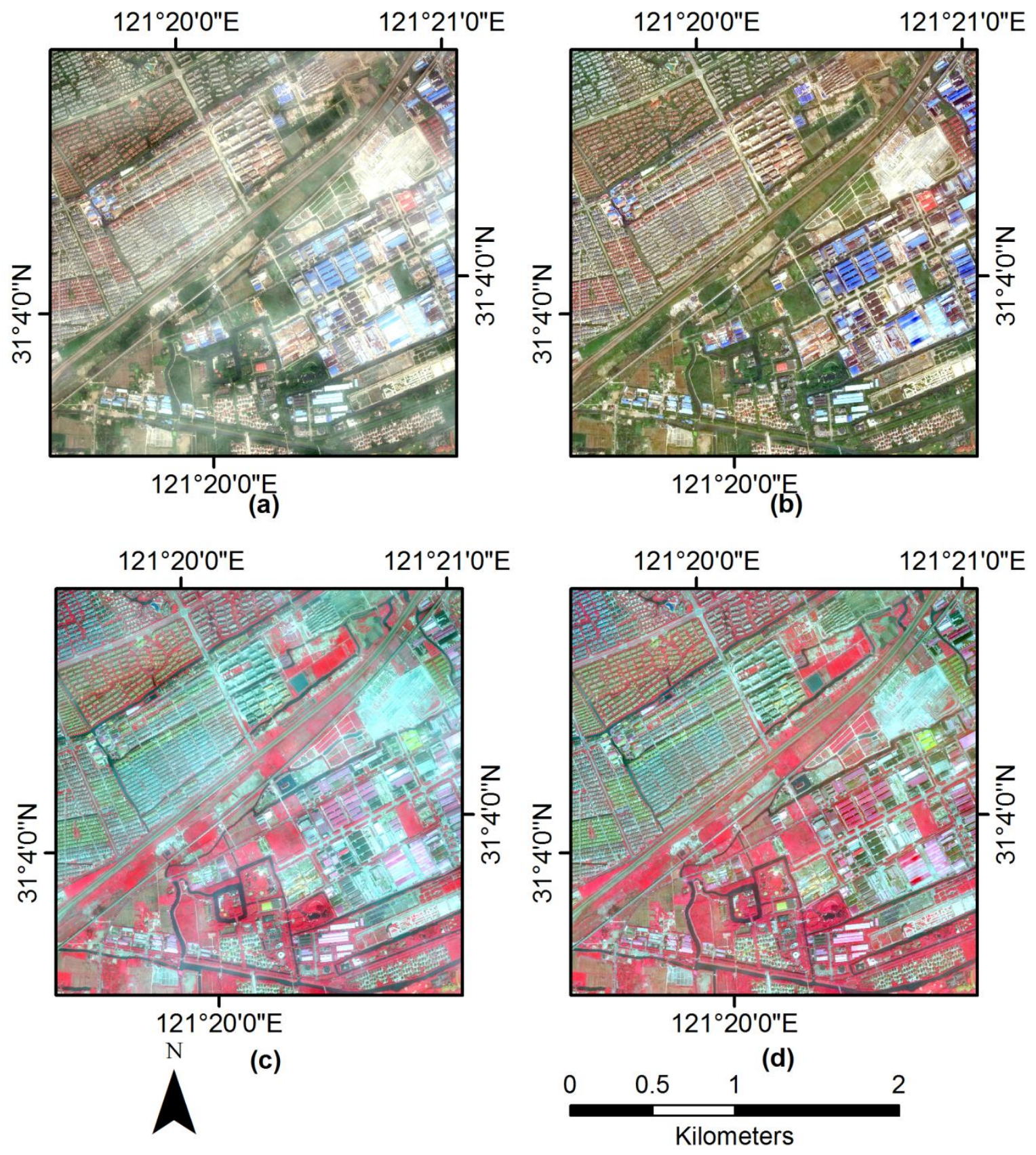

Figure S1. Haze removal performance on Worldview3 satellite imagery (Shanghai, China, GSD = $1.2 \mathrm{~m}$ ) (using 2\% linear stretch). The first row shows the true color (red, green, blue) band combination, the second row the corresponding false color (nir2, red, green) image with: (a), (c) original images; (b), (d) dehazed images by the proposed method. The visualization comparison shows that the proposed method effectively removed the haze pixels in the images and maintained the color consistency of various land cover visually. 

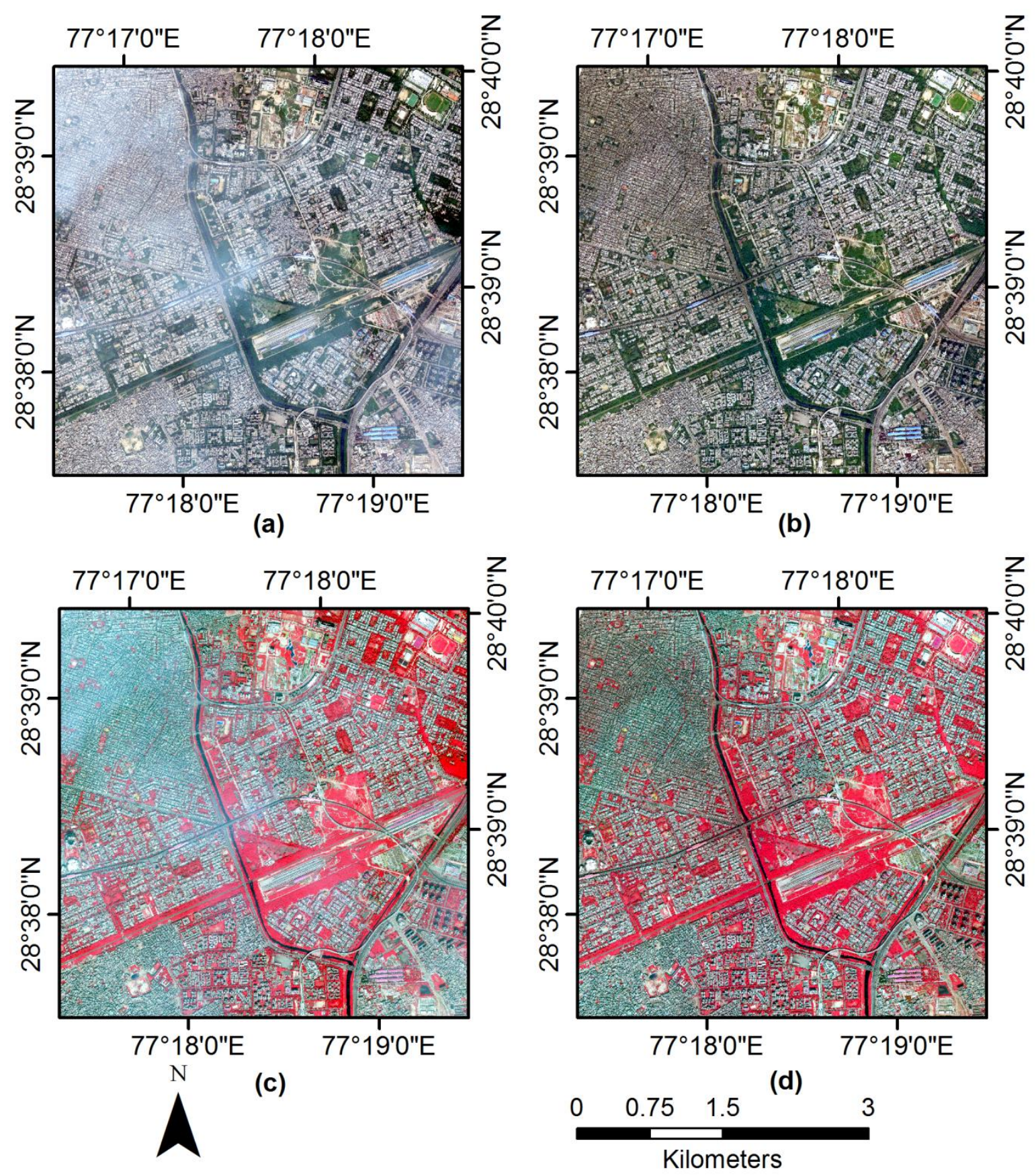

Figure S2. Haze removal performance on Worldview3 satellite imagery (New Delhi, India, GSD = $1.2 \mathrm{~m}$ ) (using $2 \%$ linear stretch). The first row shows the true color (red, green, blue) band combination, the second row the corresponding false color (nir2, red, green) image with: (a), (c) original images; (b), (d) dehazed images by the proposed method. The visualization comparison shows that the proposed method effectively removed the haze pixels in the images and maintained the color consistency of various land cover visually. 


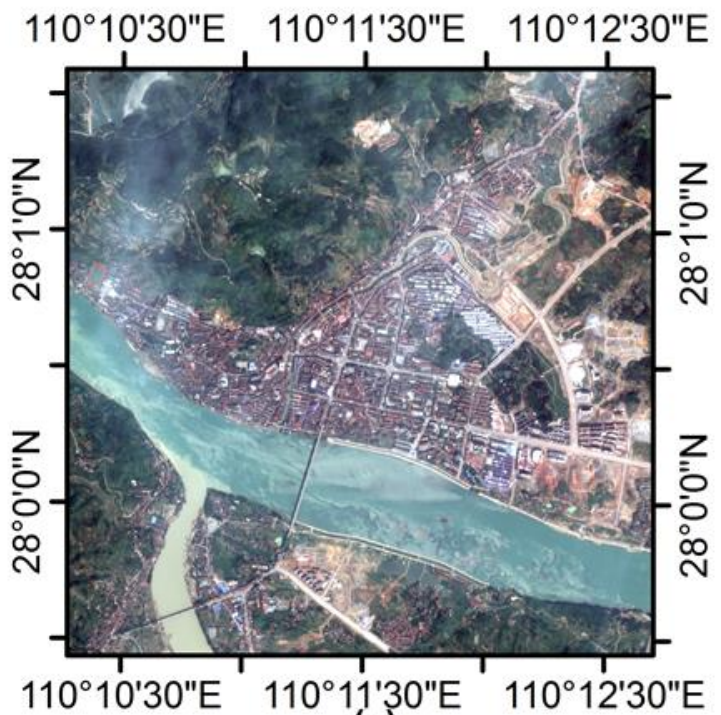

(a)

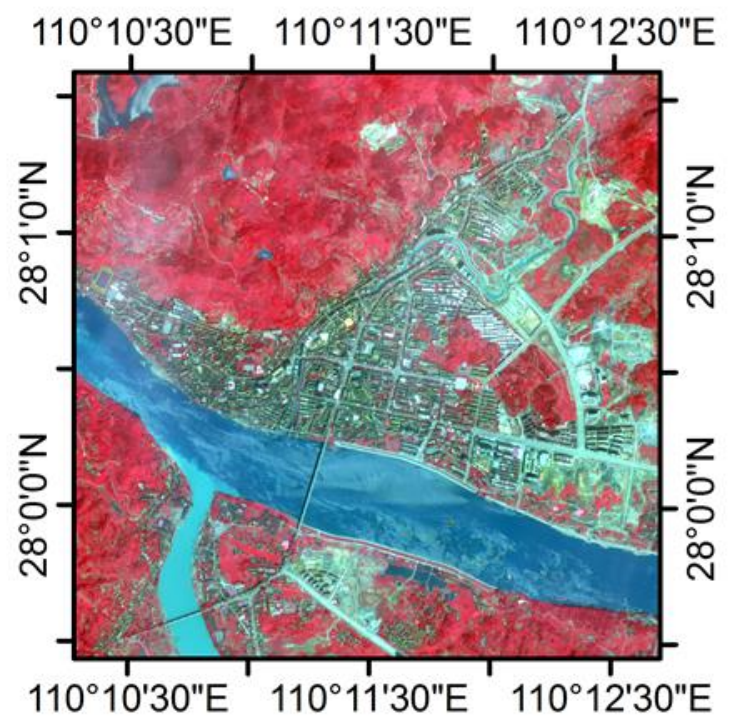

(c)

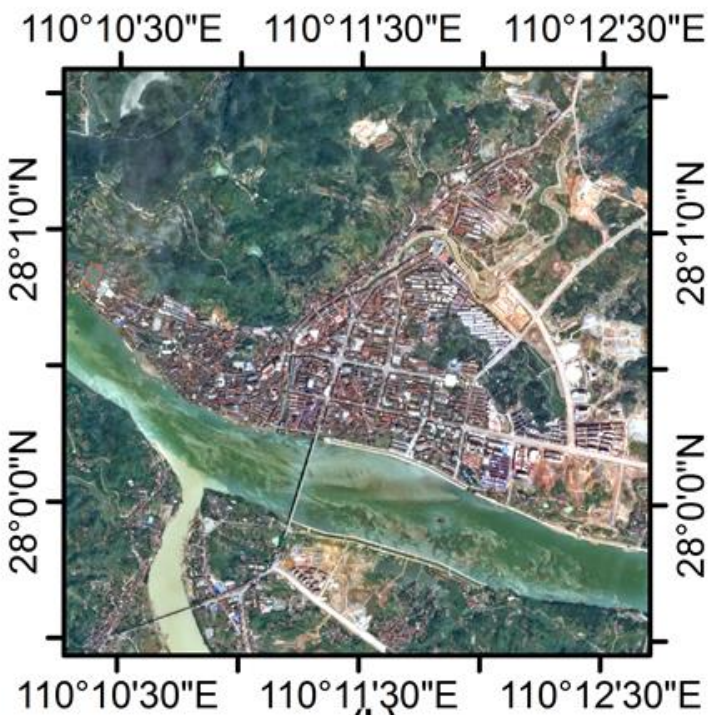

(b)
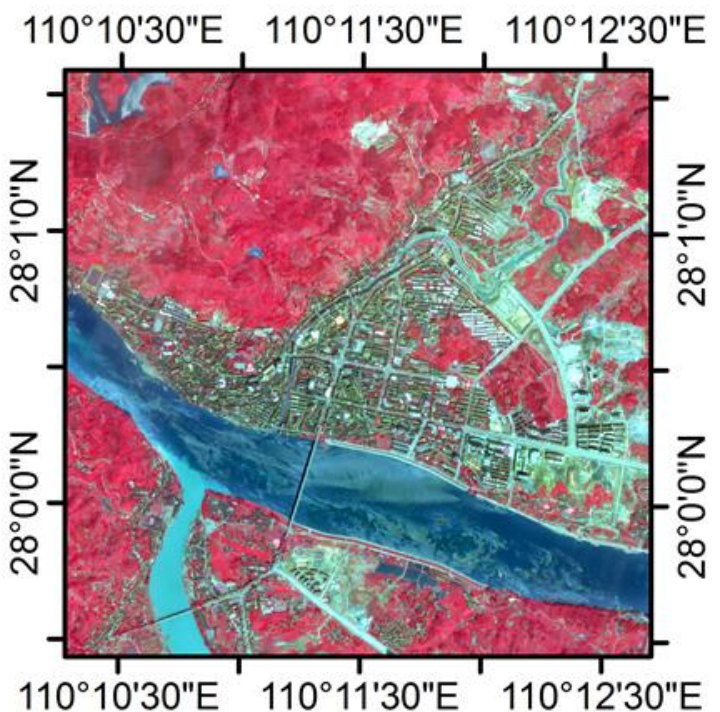

(d)
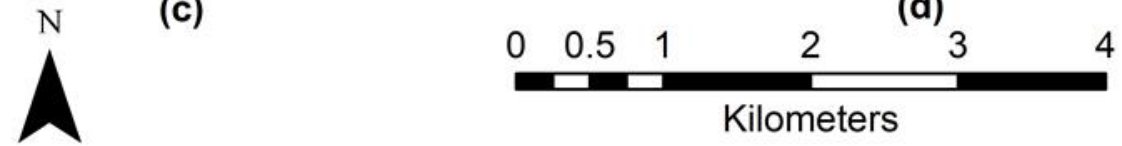

Kilometers

Figure S3. Haze removal performance on Pléiades satellite imagery (Chenxi, China, GSD = $2 \mathrm{~m}$ ) (using 2\% linear stretch). The first row shows the true color (red, green, blue) band combination, the second row the corresponding false color (nir, red, green) image with: (a), (c) original images; (b), (d) dehazed images by the proposed method. The visualization comparison shows that the proposed method effectively removed the haze pixels in the images and maintained the color consistency of various land cover visually. 


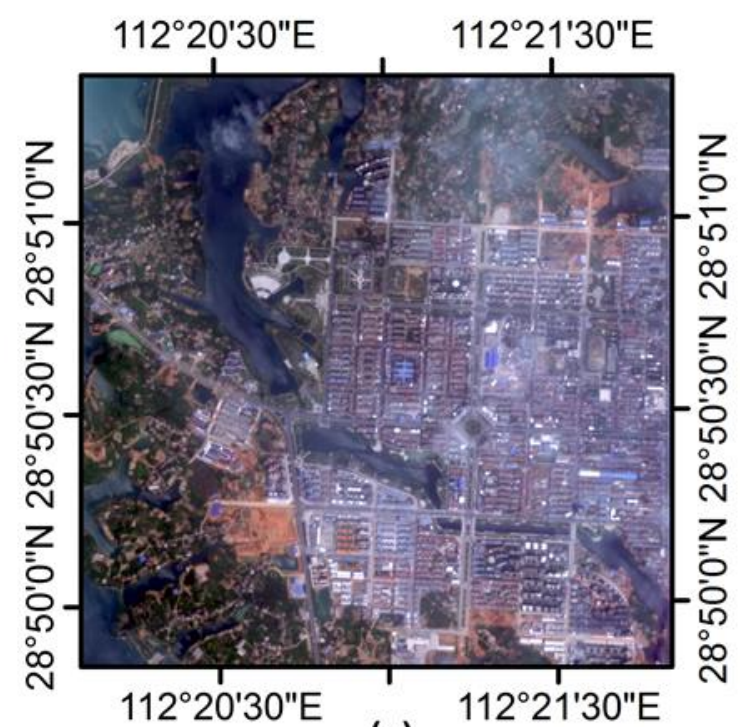

(a)

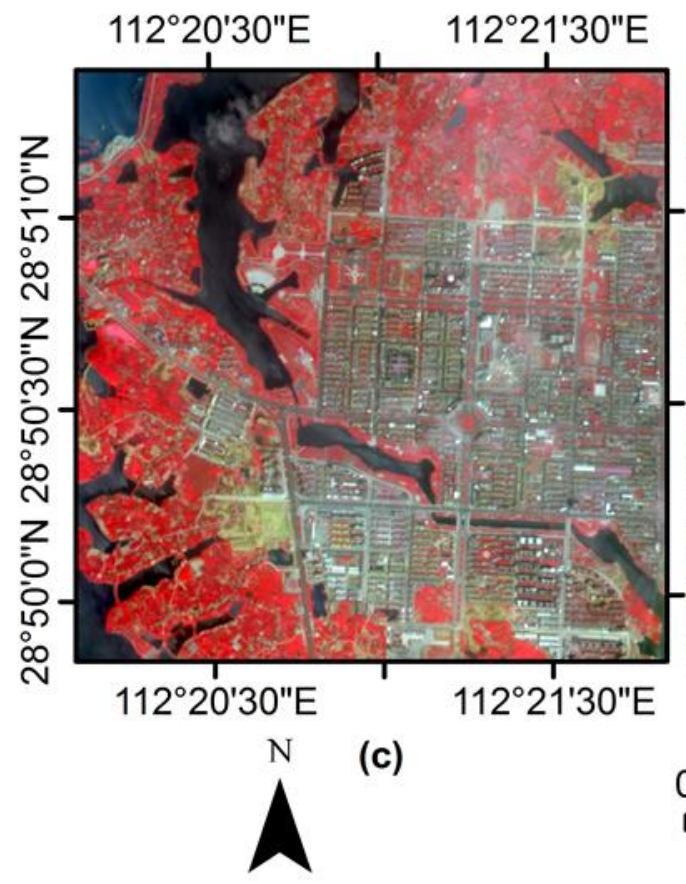

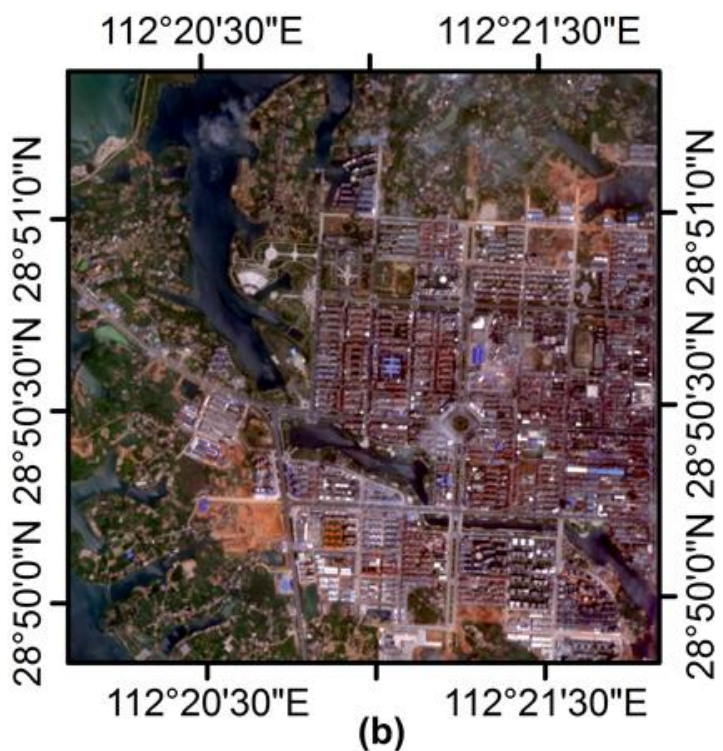

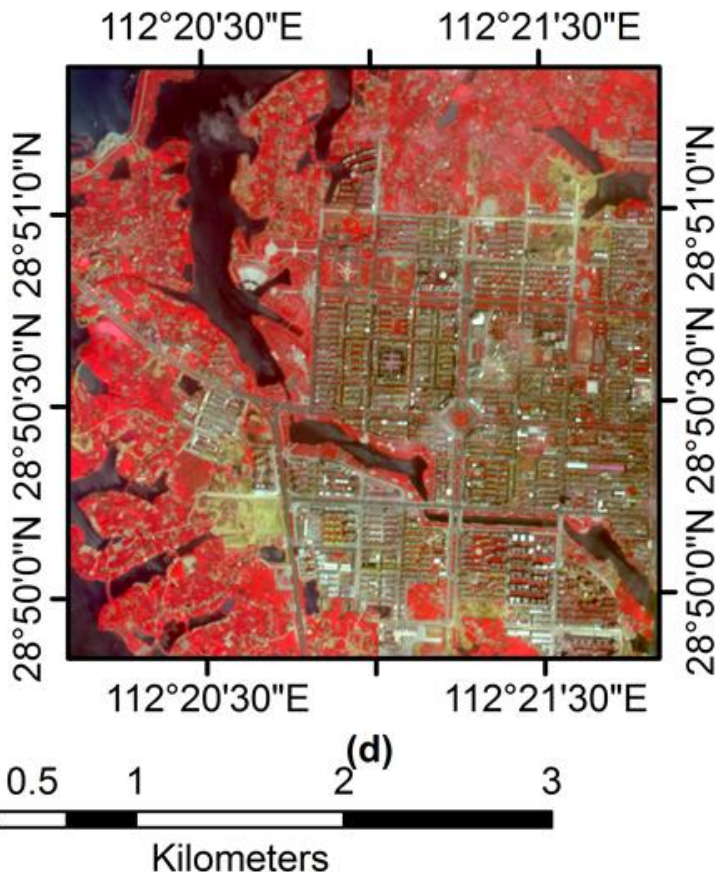

Figure S4. Haze removal performance on Gaofen-2 satellite imagery (Yuanjiang, China, GSD = 3.24 m) (using 2\% linear stretch). The first row shows the true color (red, green, blue) band combination, the second row the corresponding false color (nir, red, green) image with: (a), (c) original images; (b), (d) dehazed images by the proposed method. The visualization comparison shows that the proposed method effectively removed the haze pixels in the images and maintained the color consistency of various land cover visually. 
Annex E: Manuscript V 


\title{
A Novel Method of Panchromatic Image Weighted Multispectral Image Reconstruction
}

\author{
Dengkui Mo, Hans Fuchs, Lutz Fehrmann, Paul Magdon, Christoph Kleinn \\ Forest Inventory and Remote Sensing, Burckhardt Institute, Faculty of Forest Sciences and \\ Forest Ecology, Georg-August-Universität Göttingen \\ Büsgenweg 5, 37077 Göttingen, Germany, dmo@gwdg.de, ckleinn@gwdg.de
}

\begin{abstract}
It is a major issue to inherit spatial and spectral details from panchromatic (Pan) and multispectral (MS) images synchronously to enhance the MS image. This paper proposes a novel method of MS image reconstruction using the Pan image weighted bilateral filter (Pan-weighted) method. Compared with the traditional up-sampling interpolation methods, a Pan-weighted method helps introduce more spatial details from Pan image through weighting map characteristics and preserving spectral characteristics. Quantitative analyses and visual comparisons demonstrate the superiority of the proposed method. Further, two potential areas of application were also introduced in this paper: refinement of pansharpening and analyses of vegetation indices. The proposed method would be an alternative or an improved upsampling interpolation method, which would be a widely accepted technique for refined satellite image processing.

Index Terms-multispectral image reconstruction, upsampling, interpolation, crossbilateral filter, high-resolution satellite image, pansharpening, pan-weighted bilateral filter, edge reserving, NDVI, edge effects
\end{abstract}

\section{INTRODUCTION}

Many datasets from optical satellite remote sensing are delivered with both a high spatial resolution panchromatic image band (Pan) as well as with corresponding lower resolution multispectral bands (MS) [1]. The spatial resolution of MS bands is typically four times (e.g., Pleiades GeoEye-2, WorldView-3, or two times (Landsat-8, Sentinel-2) lower than the Pan band. Multispectral imagery with more than three bands do provide not only better fidelity for image displays but also have distinct advantages for many applications, such as identification of 
vegetation, classification of land cover and modeling of surface variables [2]. However, the coarser spatial resolution of the multispectral bands leads to more mixed pixels and more of blurred spatial features. Commonly, finer spatial features are visible only on the Pan band, but are difficult to discern on in the multispectral bands. There are significantly different spectral and spatial characteristics between the MS and Pan respectively. In the case of data transmission and storage restrictions, the current instruments are not capable of providing both spatial and spectral high-resolution images. Thus, it is particularly necessary to develop a method of integrating the advantageous characteristics of MS and Pan.

Multispectral image reconstruction, as we pursue it in this study, is characterized by the following three features:

a) the reconstructed multispectral image has the same spatial resolution as the Pan image;

b) the reconstructed multispectral image inherits the spatial details of the Pan image;

c) the reconstructed multispectral image retains the spectral characteristics of the original multispectral image.

In our study, both multispectral and Pan data are from the same satellite instrument. Methodological approaches to image interpolation and image pansharpening are widely used to reconstruct multispectral images. We describe them in some detail below.

The different spatial pixel sizes of the multispectral and Pan bands need to be brought to the same spatial resolution: techniques like the nearest neighbor, bilinear, and bicubic interpolation are commonly used to upsample the lower spatial resolution multispectral bands to the resolution of the Pan band. These techniques default in the state-of-the-art remote sensing image processing software such as ENVI 5.3 (http://www.harrisgeospatial.com). From a practical point of view, the simple interpolation techniques have the advantage that processing speed is fast while multispectral features are preserved; but they create blurred or jagged images, while not adding additional spatial details because they refer only to the neighborhood pixels of the image themselves. 


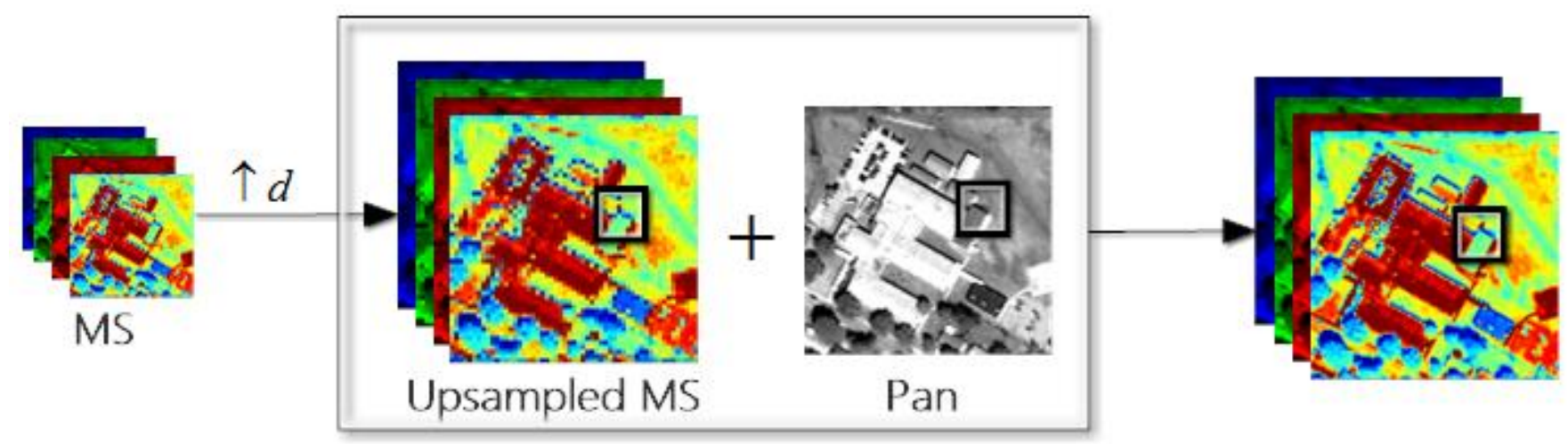

Fig. 1. The flowchart of a general pansharpening method. The multispectral image first upsampled to the image size of the panchromatic image, then extracts the spatial details of the panchromatic image and injects it into the upsampled multispectral image to get the final pan-sharpened image.

The procedure of fusing multispectral bands and the Pan band is called pansharpening. "Pansharpening" is the abbreviation of "Panchromatic sharpening", which means that the panchromatic (single band) image is being used to "sharpen" multispectral images [3], thus combining the more detailed spectral information of the multispectral bands with the more detailed spatial information of the Pan band. Pansharpening is a default step of enhancing image quality for many applications in remote sensing.

A general pansharpening framework includes two steps (see Fig. 1): a) upsampling of the multispectral bands to the finer spatial resolution of the Pan band; b) extracting and adding spatial details into upsampled MS. Many techniques of pansharpening have been proposed (see reviews in [4-8]). Also, Snehmani et al. (2016) [9] compared 27 pansharpening techniques regarding the characteristics (1) quantitative analysis, (2) visual inspection, and (3) computational complexity. Their results showed that no single pansharpening technique demonstrated promising results to simultaneously retaining both spectral and spatial characteristics.

In this paper, we introduce and test a bilateral filter technique to integrate the spectral information from multispectral bands and the spatial details from the Pan band. We also compare the performance of this novel approach to current standard approaches. 


\section{METHODS}

\section{A. Upsampling methods}

In order to reconstruct multispectral images, the multispectral image must be upsampled to the size of the corresponding panchromatic image. There are three well-known interpolation methods: nearest neighbor, bilinear interpolation, and bicubic convolution, which are widely used in a variety of commercial remote sensing software. According to their algorithms and practice, these methods have their characteristics as follows:

The nearest neighbor technique selects the nearest point, regardless of adjacent points, thus generating piecewise constant interpolation. The nearest neighbor may also be used for continuous data, but the results can be blocky.

Bilinear interpolation uses the weighted average of the nearest four points. Because values can be changed, it is not recommended to use the bilinear interpolation approach for categorical data. Instead, it is often used for continuous data, such as elevation and original slope values.

Bicubic convolution determines the output of a point from the weighted average of the 16 closest pixels and fits a smooth curve through the points to find the final value for the target pixel. The resulting image is slightly sharper than from bilinear interpolation resampling, and it does not have the disjointed appearance produced by the nearest neighbor. Similar to bilinear interpolation, cubic convolution is considered to be the most suitable for continuous data.

The three interpolation methods rely on the image itself, which easily result in blocks or blur effects on the landcover boundaries of satellite images. In this study, we first use these three interpolation methods to upsample the coarser spatial resolution of the multispectral bands to the finer resolution of the panchromatic band and then try to reconstruct the so-called accurate multispectral bands from the panchromatic band.

\section{B. Pan-weighted multispectral image reconstruction}

Bilateral filtering inspires the pan-weighted multispectral image reconstruction method proposed here. A bilateral filter is a kind of nonlinear, edge-preserving and noise-reduction smoothing filtering method [10]. The intensity value of each pixel in the image is replaced by the 
weighted average intensity value of the nearby pixel. The weight can be based on a Gaussian distribution. Crucially, weights depend not only on the Euclidean distance of the pixel but also on the difference in radiation (e.g., color intensity, depth distance, etc.). The bilateral filter preserves the sharp edges by systematically traversing each pixel and adjusting the weights of adjacent pixels accordingly.

For the new method proposed here, a modification of the traditional bilateral filter method is suggested: the weight matrix for each pixel is calculated from the panchromatic image rather than from the multispectral image itself.

Referring to and modifying [11], the pan-weighted multispectral image reconstruction is redefined for the new approach as

$$
M S_{\lambda}{ }^{\text {filtered }}(x)=\frac{1}{W_{p}} * \sum_{x_{i} \in \Omega} M S_{\lambda}\left(x_{i}\right) f_{r}\left(\left\|I\left(x_{i}\right)-I(x)\right\|\right) g_{s}\left(\left\|x_{i}-x\right\|\right)
$$

where the normalization term is

$$
W_{p}=\sum_{x_{i} \in \Omega} f_{r}\left(\left\|I\left(x_{i}\right)-I(x)\right\|\right) g_{s}\left(\left\|x_{i}-x\right\|\right)
$$

$M S_{\lambda}^{\text {filtered }}$ is the filtered $\lambda$ band spectral image;

$M S_{\lambda}$ is the original input spectral image to be filtered;

$I$ is the original panchromatic image;

$x$ are the pixel coordinates of the current pixel to be filtered;

$\Omega$ is a pixel set of a window centered on $x$;

$f_{r}$ is the range kernel used to smooth differences in intensities.

$g_{s}$ is the spatial kernel used to smooth the difference in coordinates.

Both $f_{r}$ and $g_{s}$ can be Gaussian functions;

As mentioned above, the weight $W_{p}$ is assigned using spatial distance and intensity difference. 
Consider a pixel located at $(i, j)$ which needs to be reconstructed in the image using its neighboring pixels and one of its neighboring pixels is located at $(k, l)$. Then, the weight assigned for pixel $(k, l)$ when reconstructing the pixel $(i, j)$ is given by:

$$
w_{p}(i, j, k, l)=e^{\left(-\frac{(i-k)^{2}+(j-l)^{2}}{2 \sigma_{s}^{2}}-\frac{\|I(i, j)-I(k, l)\|^{2}}{2 \sigma_{r}^{2}}\right)}
$$

where $\sigma_{s}$ and $\sigma_{r}$ are smoothing parameters, and $I(i, j)$ and $I(k, l)$ are the Pan intensity of pixels $(i, j)$ and $(k, l)$ respectively. After calculating the weights, the pixel values are normalized by

$$
M S_{\lambda}^{\text {filtered }}(i, j)=\frac{\sum_{k, l} M S_{\lambda}(k, l) * w_{p}(i, j, k, l)}{\sum_{k, l} w_{p}(i, j, k, l)}
$$

where $M S_{\lambda}^{\text {filtered }}(i, j)$ is the filtered MS intensity of the pixel $(i, j)$.

Although $\sigma_{s}$ and $\sigma_{r}$ would affect the results of multispectral image reconstruction, experiments have shown that they do not have a significant impact. In practice, we define these two parameters as follows:

The size $\mathrm{w}$ is the radius of a quadratic moving window. The default setting is $\sigma_{s}^{2}=w$, The number of pixels in the quadratic window is $n=\left(w^{*} 2+1\right)^{2}$, if $w=3, \sigma_{s}=\sqrt{3} . \sigma_{s}$ is fixed once the window is defined.

Default $\sigma_{r}^{2}(i, j)=\max (a b s(I(i, j)-I(k, l))) \cdot \sigma_{r}$ is dynamically determined from the values of the surrounding pixels.

\section{AN EMPIRICAL STUDY}

\section{A. Study site and satellite images}

The proposed algorithm does not depend on the sensor and has been implemented in a general manner. For the present study, however, we tested the new algorithms for Pléiades sensor imagery, which cover a typical landscape in rural Southeast China with urban and mountain forest areas 
(see Fig. 2). Imagery captured from September 26th, 2014, with sun azimuth $148.3^{\circ}$, and sun elevation 56.6 $6^{\circ}$ Both Pan and MS images are orthorectified with rational polynomial coefficients (RPCs) using a digital elevation model (DEM) and then calibrated to radiance. It is worth noting that all the images are co-registered.
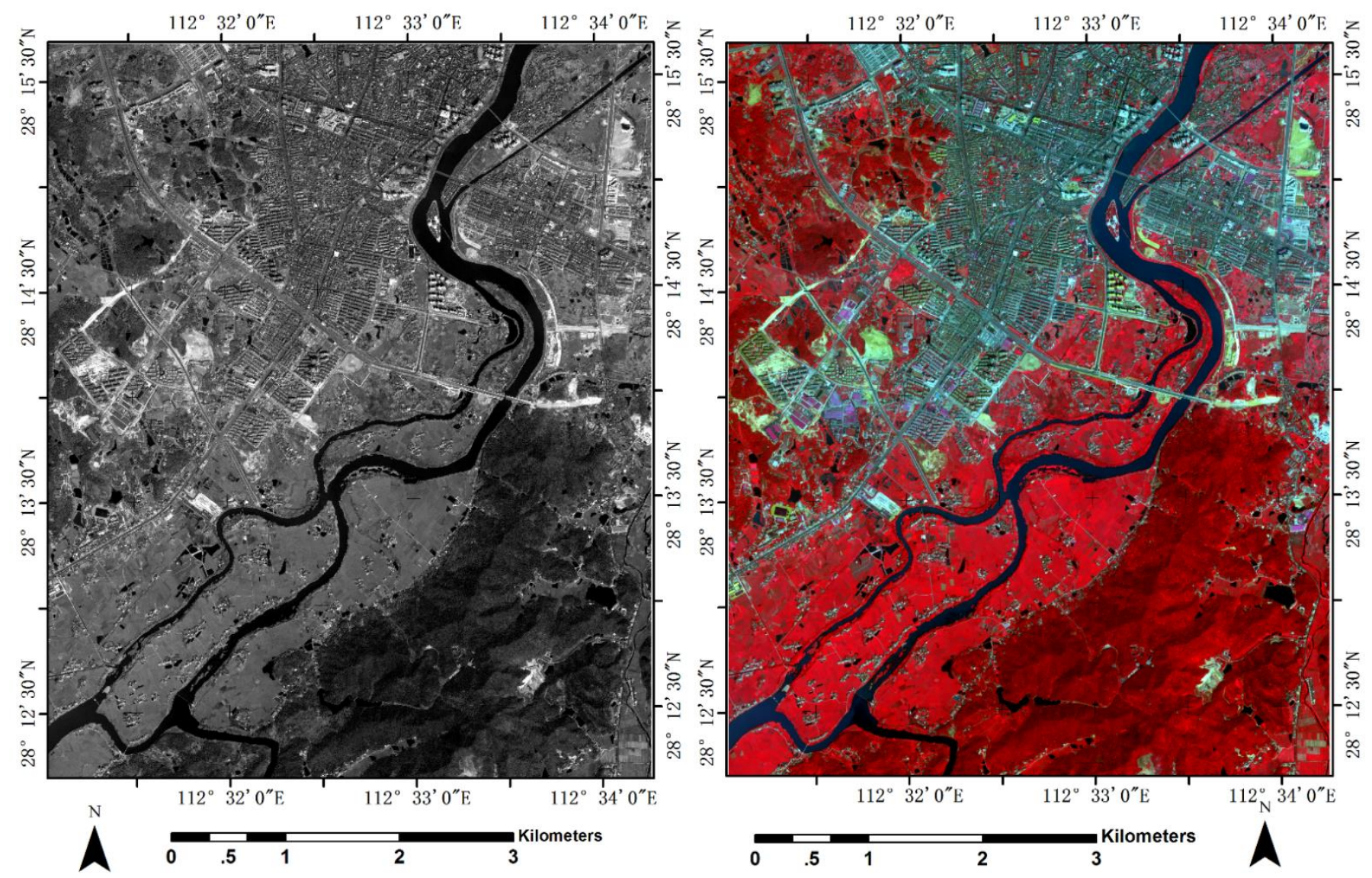

Fig. 2. Study site and Pléiades data (Ningxiang city, China). Left: Pan, $0.5 \mathrm{~m}$ spatial resolution, $12300 * 10072$ pixels, and right: MS, $2 \mathrm{~m}$ spatial resolution, $3075 * 2518$ pixels, false color composite (near-infrared, red, green).

\section{B. Experiments based on degraded images}

The goal is to build an MS image with the higher spatial resolution of the PAN band so that the resulting higher resolution MS imager is as close as possible to an image from an ideal multispectral sensor that has the spatial resolution of the Pan band. In general, this ideal multispectral image does not exist. Thus we use the Wald protocol [12] to solve this problem, including downsampling MS and Pan images, and selecting the original MS as a valid reference.

MS reconstruction and evaluation based on the Wald protocol is summarized in Fig. 3. Degraded MS and degraded Pan images are assumed to be input data for pan-weighted MS 
reconstruction, and the original MS is used as reference data. In a first step, the degraded data is resampled to higher spatial resolution using three interpolation methods, and then in a second step, we used the pan-weighted bilateral filter to reconstruct the multispectral image, and finally, we compared the traditional upwards interpolated images and pan-weighted reconstructed MS images with the reference data separately. We will explore whether the proposed methods perform better based on the visual comparison and quantitative quality analysis in the following subsections.

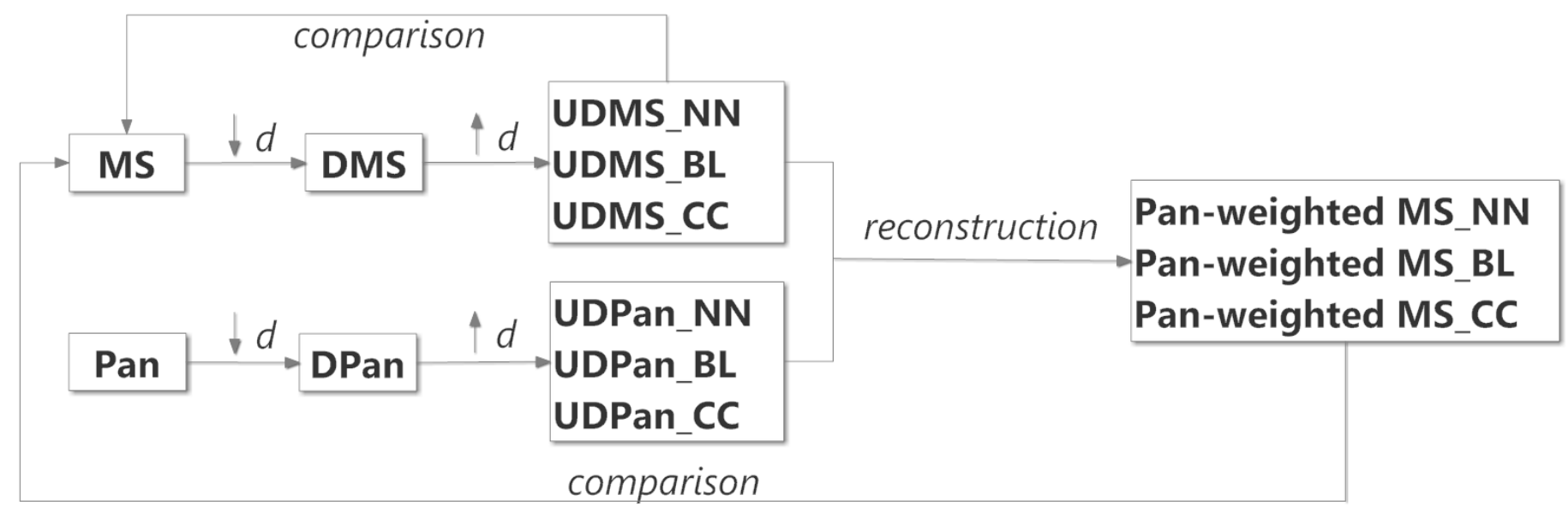

Fig. 3 Flowchart of pan-weighted multispectral image reconstruction from degraded images. DMS: degraded MS, DPan: degraded Pan, UDMS: upgraded DMS, UDPan: upgraded DPan, NN: nearest neighbor, BL: bilinear interpolation, and BC: bicubic convolution. The degrade ratio is 0.25 , and the upgrade ratio is 4 .

\section{Visual comparison}

The visual comparisons of the results using the Pleiades data for traditional interpolations and the proposed methods are shown in Fig. 6, Fig. 7, Fig. 8, and Fig. 9. For each figure here, only a subset of the data is shown so that more details can be distinguished. These four figures represent the classes such as build-up, water body, road, and bare land, respectively. The upgraded images from nearest neighbor interpolation are jagged on the boundary of land cover. The upgraded images from bilinear interpolation and bicubic convolution are blurred on the border of land cover. The reconstructed MS images are similar and close to the reference image in spectral characteristics while being spatially free of jagged and blurred effects. Compared with the MS images upgraded by the three traditional interpolation methods, visual inspection shows that the reconstructed images are more similar and stable, preserving quite well the spectral characteristics of the reference MS image and introducing the spatial details of Pan image. 


\section{Quantitative Quality Assessment}

Various quality criteria have been proposed and used to quantitatively evaluate the performance of pansharpening $[13,14]$. These quality criteria include the correlation coefficient (CC), the degree of distortion (DD) [15], the root-mean-square error (RMSE), the erreur relative globale adimensionnelle de synthese (ERGAS (erreur relative globale adimensionalle de synthèse), relative dimensionless global error in synthesis in English) [16], and the spectral angle mapper (SAM). These quality criteria used in this paper are defined as follows.

RMSE is often used to compare the differences between two images by directly calculating the change in pixel values. It is defined as

$$
\mathrm{RMSE}=\sqrt{\frac{1}{N} \sum_{i=1}^{N}\left(X_{i}-\hat{X}_{i}\right)^{2}}
$$

$\hat{X}_{i}$ is the pixel value of the original image $\hat{X}$, and $X_{i}$ is the pixel value of the pan-sharpened image $X . N$ is the number of pixels of the MS image. The upsampled image is closer to the original image when RMSE is smaller.

CC is the most popular similarity criterion. The more similar the two images are, its value closer to 1 .

$$
C C=\frac{\sum_{i}^{N}\left[\left(X_{i}-\bar{x}\right) \cdot\left(\hat{X}_{i}-\hat{x}\right)\right]}{\sqrt{\sum_{i}^{N}\left[\left(X_{i}-\bar{x}\right)^{2}\right] \cdot \sum_{i}^{N}\left[\left(\hat{X}_{i}-\hat{x}\right)^{2}\right]}}
$$

DD directly reflects the level of reconstructed image distortion. It is defined as

$$
D D=\frac{1}{N} \sum_{i=1}^{N}\left|X_{i}-\hat{X}_{i}\right|
$$

ERGAS reflects the overall quality of the reconstructed image. It represents the difference between translation and the original image, and it is defined as

$$
\mathrm{ERGAS} \triangleq \frac{100}{R} \sqrt{\frac{1}{K} \sum_{k=1}^{K}\left(\frac{\mathrm{rmse}(k)}{\mu(k)}\right)^{2}}
$$


where $K$ is the total number of bands, $R$ is the scale ratio, $\operatorname{rmse}(k)$ is the root-mean-square error between the $k^{\prime} t h$ fused band and the reference band, and $\mu(k)$ is the mean of the $k^{\prime} t h$ band. A small ERGAS value means small spectral distortion so that the algorithm has high preservation of spectral information. ERGAS is a measure of global radiometric distortion of the reconstructed images. Smaller ERGAS means the better performance of multispectral reconstructions.

SAM calculates the angle between the true and estimated spectral vectors. It is performed on a pixel-by-pixel base. SAM is measured in degrees or radians, usually averaged over the entire image to produce global measurements of spectral distortion. If the angle is $0^{\circ}$, this means no spectral change.

$$
\operatorname{SAM}\left(\mathbf{X}_{i}, \hat{X}_{i}\right)=\arccos \left(\frac{\left\langle\mathbf{X}_{i}, \hat{X}_{i}\right\rangle}{\left\|\mathbf{X}_{i}\right\|_{2}\left\|\hat{X}_{i}\right\|_{2}}\right)
$$

In addition to evaluating multispectral images, we are also interested in the performance of Normalized Difference Vegetation Index (NDVI) reconstruction. NDVI is the normalized difference between the brightness values of the near-infrared (NIR) and red channels per pixel:

$$
\mathrm{NDVI}=\frac{(\mathrm{NIR}-\mathrm{Red})}{(\mathrm{NIR}+\mathrm{Red})}
$$

Tables I and II show the results of the quantitative quality evaluation for different upsampling algorithms for multispectral images and NDVI images.

Table 1 summarizes the calculated assessment criteria value for the multispectral images. The second row gives the reference values of different criteria. It is evident that reconstructed multispectral images give better performance in all metrics, except that all the SAM is equal to 0 degrees.

Table 2 summarizes the calculated assessment criteria value for the NDVI images. The second row gives the reference values of different criteria. It is evident that reconstructed NDVI images give better performance in all metrics. 
From Tables I and II, in this experiment, we can see that the image reconstruction based on bilinear interpolation achieves the best performance whether for multispectral images or NDVI images.

Table 1 Quality Metrics for the multispectral images

\begin{tabular}{|l|r|r|r|r|r|}
\hline & \multicolumn{1}{l|l|}{ RMSE } & \multicolumn{1}{l|}{ CC } & \multicolumn{1}{l|}{ DD } & \multicolumn{1}{l|}{ ERGAS } & \multicolumn{1}{l|}{ SAM () } \\
\hline MS & 0 & 1 & 0 & 0 & 0 \\
\hline MS_NN & 5.7469 & 0.9385 & 3.2001 & 2.517 & 0 \\
\hline MS_NN_Reconstructed & 4.5722 & 0.9608 & 2.6867 & 2.001 & 0 \\
\hline MS_BL & 4.7185 & 0.9582 & 2.7734 & 2.0656 & 0 \\
\hline MS_BL_Reconstructed & $\mathbf{4 . 4 2 1 3}$ & $\mathbf{0 . 9 6 3 4}$ & $\mathbf{2 . 6 4 9 8}$ & $\mathbf{1 . 9 3 5 1}$ & 0 \\
\hline MS_BC & 4.9668 & 0.9539 & 2.9029 & 2.1737 & 0 \\
\hline MS_BC_Reconstructed & 4.4904 & 0.9623 & 2.6631 & 1.9648 & 0 \\
\hline
\end{tabular}

Note: Except for SAM () column, the best results are denoted using bold font.

Table 2 Quality Metrics for the NDVI images

\begin{tabular}{|l|r|r|r|r|r|}
\hline & \multicolumn{1}{l}{ RMSE } & \multicolumn{1}{l|}{ CC } & \multicolumn{1}{l|}{ DD } & \multicolumn{1}{l|}{ ERGAS } & \multicolumn{1}{l|}{ SAM () } \\
\hline NDVI & 0 & 1 & 0 & 0 & 0 \\
\hline NDVI_NN & 0.0794 & 0.9169 & 0.0485 & 16.0656 & 15.8453 \\
\hline NDVI_NN_Reconstructed & 0.0641 & 0.9446 & 0.0416 & 12.9705 & 13.7441 \\
\hline NDVI_BL & 0.0656 & 0.9419 & 0.0425 & 13.2754 & 14.0385 \\
\hline NDVI_BL_Reconstructed & $\mathbf{0 . 0 6 2 3}$ & $\mathbf{0 . 9 4 7 6}$ & 0.0413 & $\mathbf{1 2 . 6 1 5 5}$ & NaN \\
\hline NDVI_BC & 0.0688 & 0.9367 & 0.0443 & 13.9262 & 14.4096 \\
\hline NDVI_BC_Reconstructed & 0.0632 & 0.9462 & $\mathbf{0 . 0 4 1 2}$ & 12.7838 & 13.59 \\
\hline
\end{tabular}

Note: Note: Except for SAM () column, the best results are denoted using bold font. "NaN" is the result of the calculation using the same algorithm, we ignore it directly here.

\section{POTENTIAL APPLICATIONS}

As the approach proposed in this study is essentially an improved upsampling approach, the subsequent remote sensing image processing can be performed such as band math, pansharpening, image classification, and so on. Here, we demonstrate the results of applications of the refined NDVI and the pan-sharpened images visually.

\section{A. Refined NDVI images}

In general, vegetation indices are computed directly from the original MS image. Its drawback is that vegetation pixels below the spatial resolution of MS images are difficult to detect, or 
vegetation boundaries of the MS image are inconsistent with the boundaries of the Pan image. The Pan-weighted multispectral reconstruction will allow achieving higher spatial resolution of vegetation indices. Here we only select NDVI images to validate the performance of the proposed method visually.

Fig. 10 to Fig. 13illustrate that one kind of NDVI image is blocky when nearest neighbor upsampling is used, and the other two kinds of NDVI images are blurred when bilinear and bicubic upsampling approaches are used, especially along object boundaries. In contrast, the NDVI images reconstructed over Pan-weighted multispectral reconstruction are more consistent and spatially matched better with panchromatic images, regardless of the upsampling method. In addition, textures of the reconstructed NDVI image and the corresponding panchromatic image texture are closer, and the rendering colors for the reconstructed NDVI image and the original NDVI image remain consistent.

\section{B. Refined pansharpened images}

Even though many pansharpening techniques are described in the literature [5-7, 2], Here we only select Brovey sharpening to validate the performance of the proposed method visually. Brovey is widely introduced in a variety of commercial remote sensing image processing software. Brovey assumes that a panchromatic image spans the same spectral range as the multispectral image and it is developed to increase the contrast in the high and low ends of the data's histogram [23]. Each band in the multispectral image is multiplied by a ratio of the corresponding panchromatic data divided by the sum of the multispectral bands.

From Fig. 4 and Fig. 5, we found that pansharpened images are blocky under Brovey using the nearest neighbor resampling and three traditional methods without multispectral image reconstruction have failed to handle boundary transitions, especially in the boundary area where there are significant spectral differences. In contrast, the results of Brovey sharpening with multispectral image reconstruction are more consistent and spatially matched with panchromatic images, regardless of the upsampling method applied. 


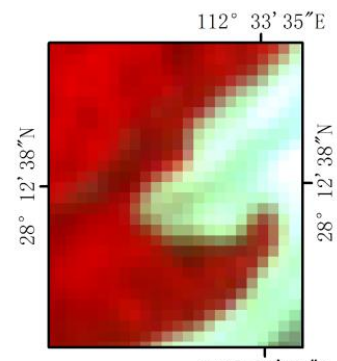

(1)

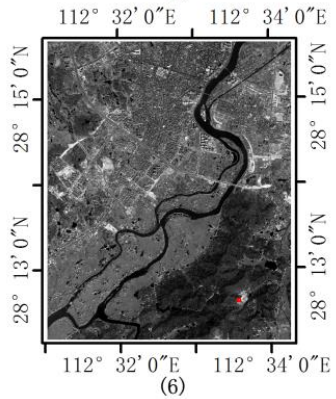

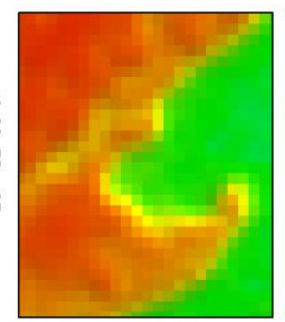

(2)

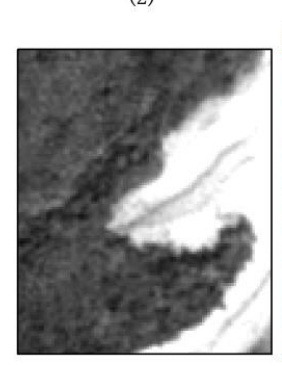

(7)
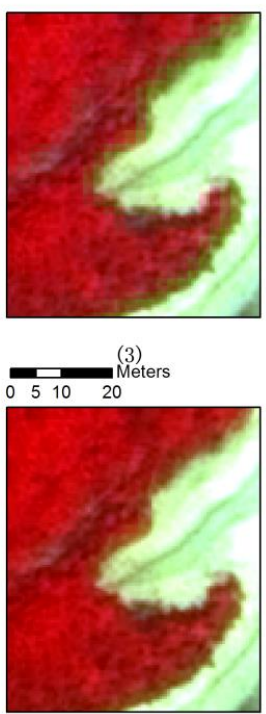

(8)

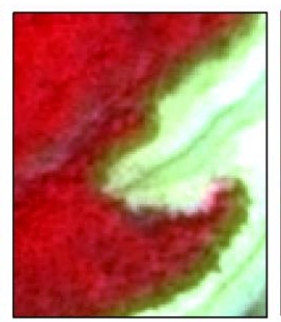

(4)

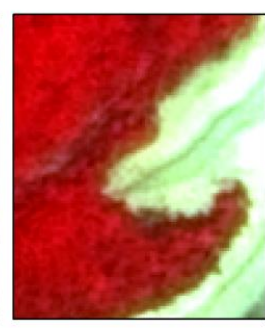

(9)

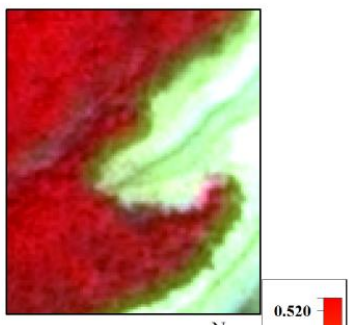

(5)

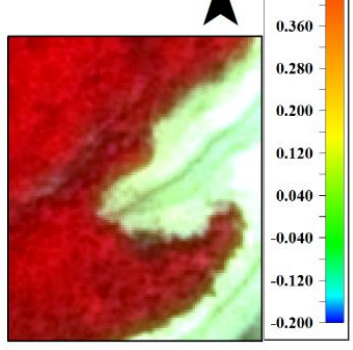

(10)

Fig. 4 Visual comparison of Brovey pansharpening results. (1) Original multispectral image, (2) NDVI image from the original MS image. (3, 4, and 5) Pansharpened images using nearest neighbor, bilinear interpolation, and bicubic convolution, respectively. (6) The red rectangle is the position of the test data. (7) Original panchromatic image. (8, 9, and 10) Pansharpened images from reconstructed images by proposed method using nearest neighbor, bilinear interpolation, and bicubic convolution, respectively, their boundary pixels are more consistent with (7). Note: For better visualization, NDVI image is simulated as terrain illumination (sun elevation $=60^{\circ}$, and sun azimuth $=45^{\circ}$ ).

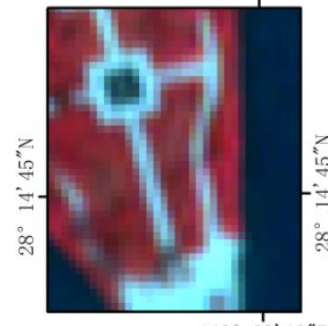

(1)

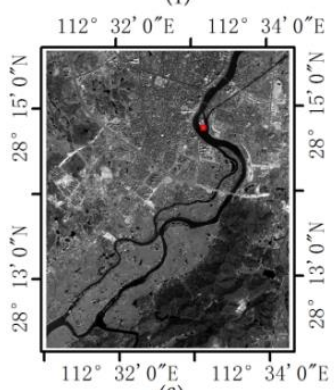

(6)

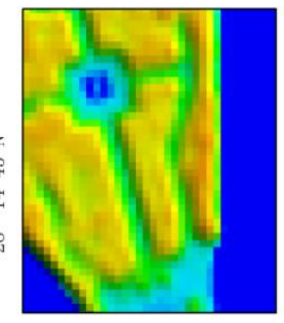

(2)

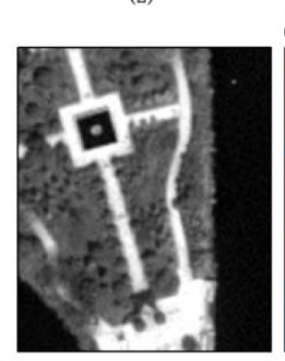

(7)
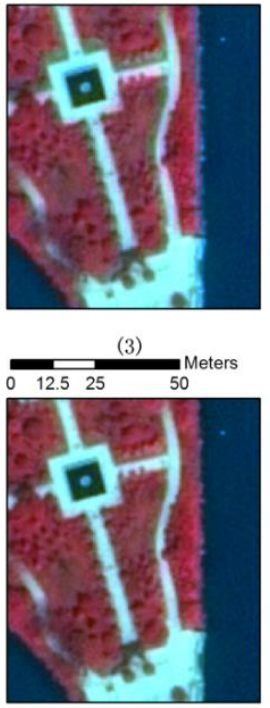

(8)

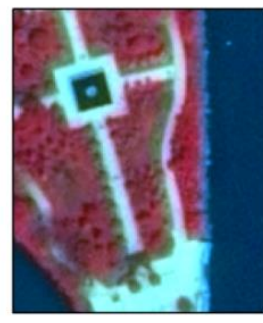

(4)

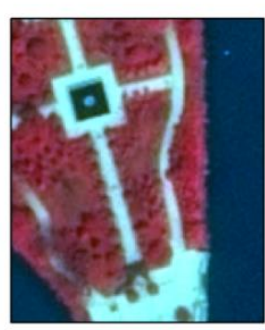

(9)

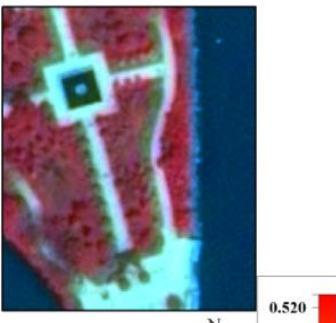

(5)

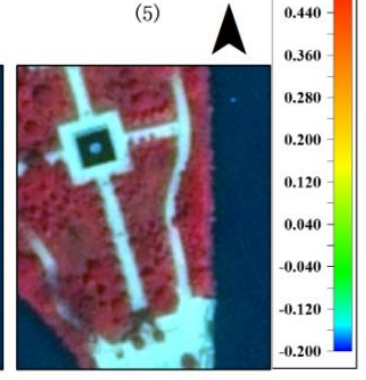

(10)

Fig. 5 Visual comparison of Brovey pansharpening results. (1) Original multispectral image, (2) NDVI image from the original MS image. (3, 4, and 5) Pansharpened images using nearest neighbor, bilinear interpolation, and bicubic convolution, respectively. (6) The red rectangle is the position of the test data. (7) Original panchromatic image. (8, 9, and 10) Pansharpened images from reconstructed images by proposed method using nearest neighbor, bilinear interpolation, and bicubic convolution, respectively, their boundary pixels are more consistent with (7). Note: For better visualization, NDVI image is simulated as terrain illumination (sun elevation $=60^{\circ}$, and sun azimuth $=45^{\circ}$ ). 


\section{CONCLUSION AND DISCUSSIONS}

A novel and straightforward method of pan-weighted multispectral image reconstruction was proposed in this study. Bilateral filter or joint bilateral filter inspire our method. In fact, such methods are widely used in other research fields - but rarely in remote sensing image processing. Its characteristic is that for each pixel of the upsampled MS image, the spatial and spectral distances of the neighborhood pixels of Pan and MS to their central pixels are introduced to compute the weight matrix. Thus, the method is naturally integrated into the spatial details and spectral characteristics of Pan and MS images. Both visual and quantitative assessments of our experimental results using Pléiades data indicate that the proposed method achieves better performance than traditional image interpolation methods. Moreover, it also achieves better performance in the post-processing of remote sensing images, such as pansharpening and refinement of vegetation index. It shows more possibilities for improved applications such as image classification and vegetation extraction and so on.

The proposed method operates locally and does not rely on global optimization. Thus it is natural that our algorithms allow for quick processing on multi-core devices and significantly reduce processing time. It is also a sensor-independent method that can be applied to any satellite image.

\section{ACKNOWLEDGMENT}

This study was supported by the German Federal Ministry of Education and Research (Project Lin4Carbon, Grant No. 033L049C), and the National Natural Science Foundation of China (Grant No. 31470643 and 31100412). The authors would like to thank Dr. Haijun Yang for technical suggestions.

Author Contributions: Dengkui Mo, Hans Fuchs, and Lutz Fehrmann designed the study. Dengkui Mo designed and developed the algorithm in the manuscript. Dengkui Mo, Hans Fuchs, and Lutz Fehrmann completed the calculations, map production, and the image quality assessment. Dengkui Mo completed the first version of the draft. Hans Fuchs, Lutz Fehrmann, and Christoph Kleinn revised the manuscript.

Conflicts of Interest: The authors declare no conflict of interest.

\section{REFERENCES}


[1] W. Sun, B. Chen, and D. W. Messinger, "Nearest-neighbor diffusion-based pansharpening algorithm for spectral images," Optical Engineering, vol. 53, no. 1, pp. 013107-013107, 2014.

[2] Y. Monno, M. Tanaka, and M. Okutomi, "Multispectral demosaicking using adaptive kernel upsampling," in Image Processing (ICIP), 2011 18th IEEE International Conference on, 2011, pp. 3157-3160: IEEE.

[3] H. R. Ghodekar and A. Deshpande, "Satellite Multispectral Image Enhancement based on Pan-sharpening using NSC-Transform," image, vol. 4, no. 10, 2016.

[4] Y. Zhang and R. K. Mishra, "A review and comparison of commercially available pansharpening techniques for high resolution satellite image fusion," in Geoscience and Remote Sensing Symposium (IGARSS), 2012 IEEE International, 2012, pp. 182-185: IEEE.

[5] A. Garzelli, F. Nencini, L. Alparone, B. Aiazzi, and S. Baronti, "Pan-sharpening of multispectral images: a critical review and comparison," in Geoscience and Remote Sensing Symposium, 2004. IGARSS'04. Proceedings. 2004 IEEE International, 2004, vol. 1: IEEE.

[6] H. Ghassemian, "A review of remote sensing image fusion methods," Information Fusion, vol. 32, pp. 75-89, 2016.

[7] L. Loncan et al., "Hyperspectral pansharpening: A review," IEEE Geoscience and remote sensing magazine, vol. 3, no. 3, pp. 27-46, 2015.

[8] Y. Qu, H. Qi, B. Ayhan, C. Kwan, and R. Kidd, "Does Multispectral/Hyperspectral Pansharpening Improve the Performance of Anomaly Detection?," in IEEE International Geoscience and Remote Sensing Symposium (IGARSS), 2017, pp. 6130-6133.

[9] Snehmani, A. Gore, A. Ganju, S. Kumar, and P. Srivastava, "A comparative analysis of pansharpening techniques on QuickBird and WorldView-3 images," Geocarto International, pp. 1-17, 2016.

[10] C. Tomasi and R. Manduchi, "Bilateral filtering for gray and color images," in Computer Vision, 1998. Sixth International Conference on, 1998, pp. 839-846: IEEE.

[11] V. V. Reddy, S. R. Kumar, and G. H. Krishna, "Guided Image Filtering for Image Enhancement," International Journal of Research Studies in Science, Engineering and Technology, vol. 1, no. 9, pp. 134-138, 2014.

[12] L. Wald, T. Ranchin, and M. Mangolini, Fusion of satellite images of different spatial resolutions: Assessing the quality of resulting images. 1997, pp. 691-699.

[13] L. Wald, "Quality of high resolution synthesised images: Is there a simple criterion?," in Third conference" Fusion of Earth data: merging point measurements, raster maps and remotely sensed images", 2000, pp. 99-103: SEE/URISCA.

[14] Q. Du, N. H. Younan, R. King, and V. P. Shah, "On the performance evaluation of pansharpening techniques," IEEE Geoscience and Remote Sensing Letters, vol. 4, no. 4, pp. 518-522, 2007.

[15] X. X. Zhu and R. Bamler, "A sparse image fusion algorithm with application to pansharpening," IEEE transactions on geoscience and remote sensing, vol. 51, no. 5, pp. 2827-2836, 2013.

[16] D. Renza, E. Martinez, and A. Arquero, "A new approach to change detection in multispectral images by means of ERGAS index," IEEE Geoscience and Remote Sensing Letters, vol. 10, no. 1, pp. 76-80, 2013. 
[17] G. D. Robinson, H. N. Gross, and J. R. Schott, "Evaluation of two applications of spectral mixing models to image fusion," Remote Sensing of Environment, vol. 71, no. 3, pp. 272281, 2000.

[18] K. Kotwal and S. Chaudhuri, "An optimization-based approach to fusion of hyperspectral images," IEEE Journal of Selected Topics in Applied Earth Observations and Remote Sensing, vol. 5, no. 2, pp. 501-509, 2012.

[19] J. Lee and C. Lee, "Fast and efficient panchromatic sharpening," IEEE transactions on geoscience and remote sensing, vol. 48, no. 1, pp. 155-163, 2010.

[20] T.-M. Tu, C.-L. Hsu, P.-Y. Tu, and C.-H. Lee, "An adjustable pan-sharpening approach for IKONOS/QuickBird/GeoEye-1/WorldView-2 imagery," IEEE Journal of Selected Topics in Applied Earth Observations and Remote Sensing, vol. 5, no. 1, pp. 125-134, 2012.

[21] J. Yang, J. Zhang, H. Li, Y. Sun, and P. Pu, Pixel level fusion methods for remote sensing images: A current review. na, 2010.

[22] Y. Zhang and R. K. Mishra, "A review and comparison of commercially available pansharpening techniques for high resolution satellite image fusion," in 2012 IEEE International Geoscience and Remote Sensing Symposium, 2012, pp. 182-185.

[23] J. Vrabel, "Multispectral imagery band sharpening study," Photogrammetric Engineering and Remote Sensing, vol. 62, no. 9, pp. 1075-1084, 1996. 

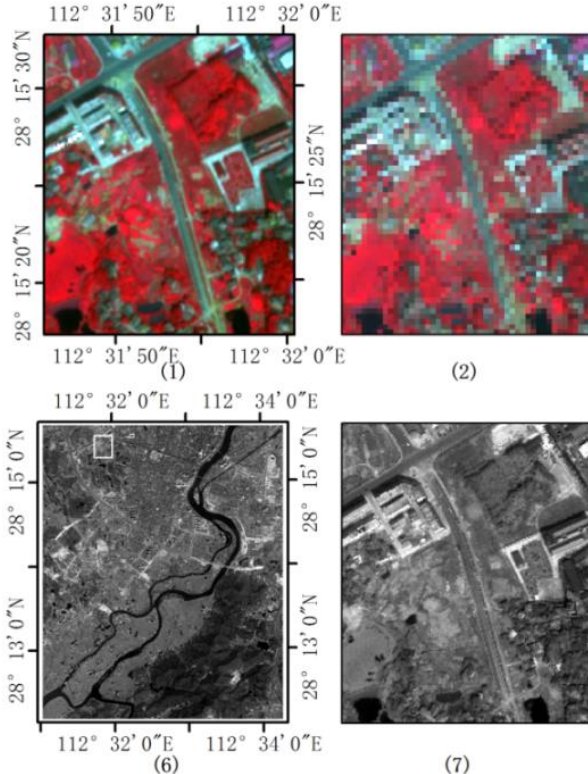

(7)
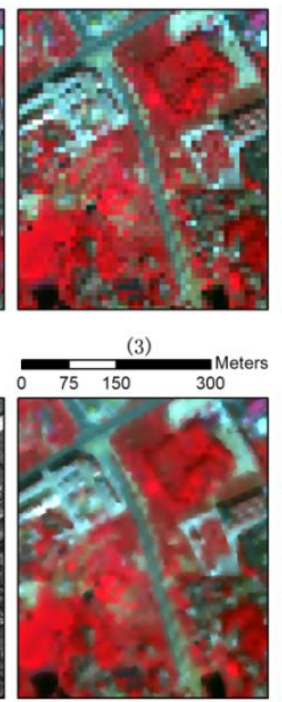

(8)

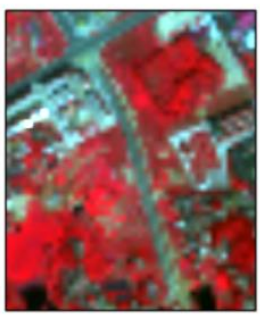

(4)

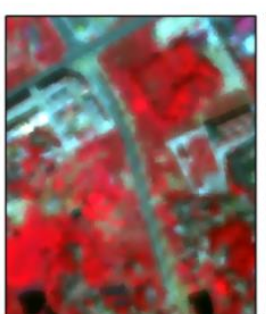

(9)
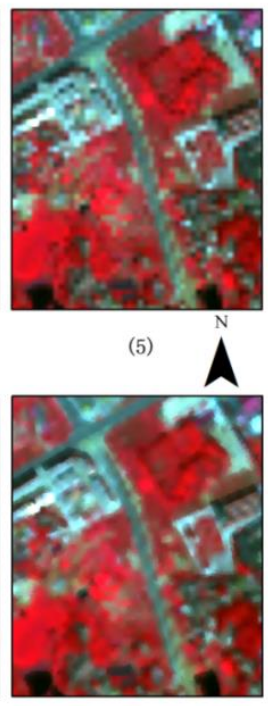

(10)

Fig. 6 (1) Original multispectral image as a reference with a spatial resolution of $2 \mathrm{~m}$. (2) Degraded multispectral image from (1) using nearest neighbor interpolation with a spatial resolution of $8 \mathrm{~m}$. (3, 4, and 5) Upgraded multispectral images from (2) using nearest neighbor, bilinear interpolation, and bicubic convolution, respectively. (6) The white rectangle is the position of the test data. (7) Degraded panchromatic image with a spatial resolution of $2 \mathrm{~m}$. (8, 9, and 10) Multispectral images reconstructed by proposed method from (7) and (3, 4, and 5) with a spatial resolution of $2 \mathrm{~m}$.
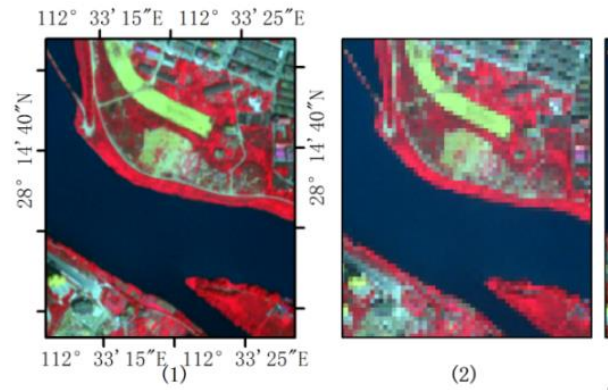

(2)
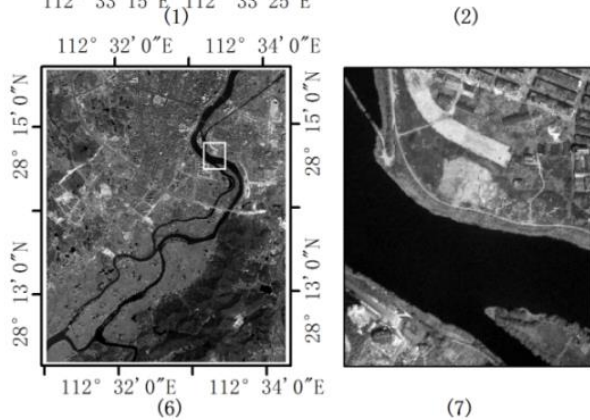

(7)
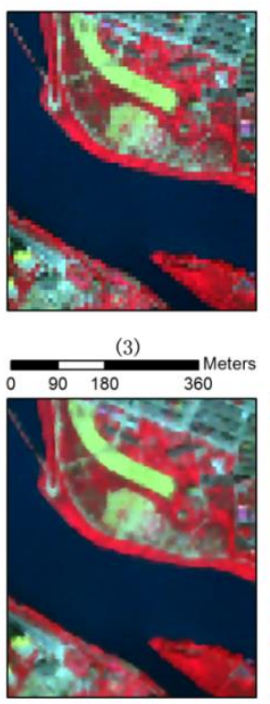

(8)

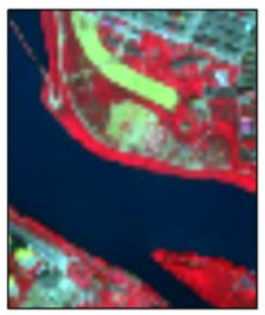

(4)

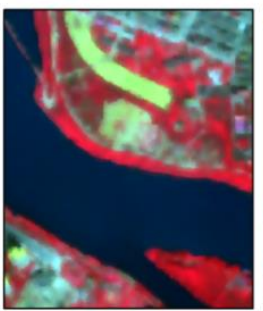

(9)

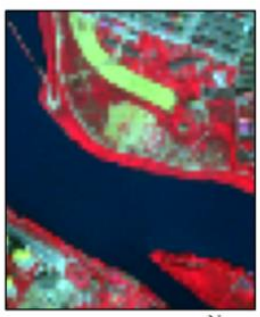

(5)

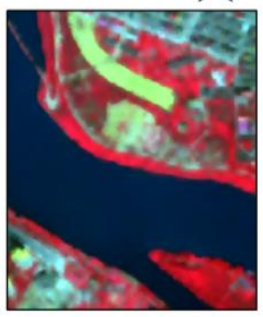

(10)

Fig. 7 (1) Original multispectral image as a reference with a spatial resolution of $2 \mathrm{~m}$. (2) Degraded multispectral image from (1) using nearest neighbor interpolation with a spatial resolution of $8 \mathrm{~m}$. (3, 4, and 5) Upgraded multispectral images from (2) using nearest neighbor, bilinear interpolation, and bicubic convolution, respectively. (6) The white rectangle is the position of the test data. (7) Degraded panchromatic image with a spatial resolution of $2 \mathrm{~m}$. (8, 9, and 10) Multispectral images reconstructed by proposed method from (7) and (3, 4, and 5) with a spatial resolution of $2 \mathrm{~m}$. 

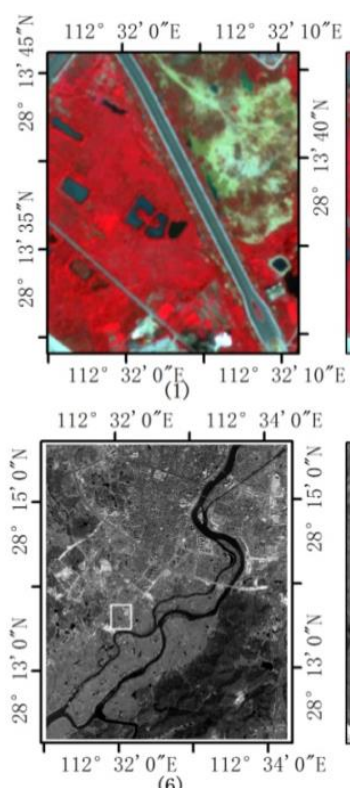

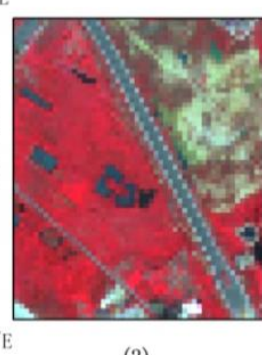

(2)

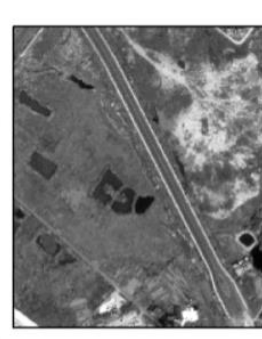

(7)
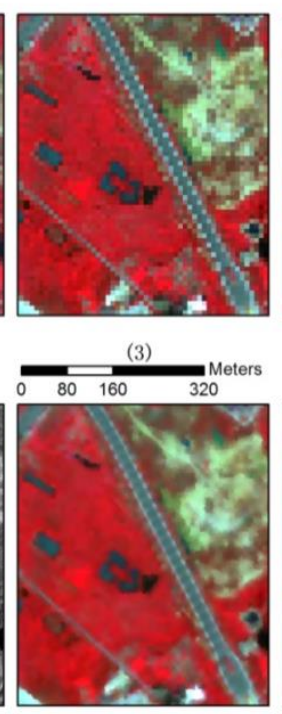

(8)

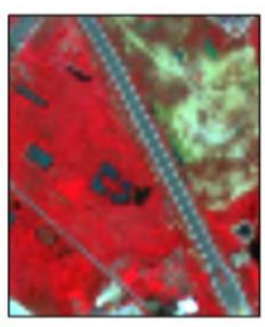

(4)

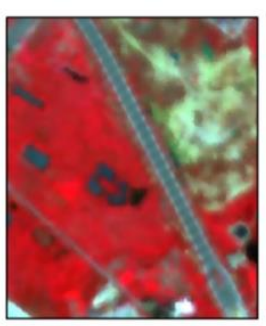

(9)

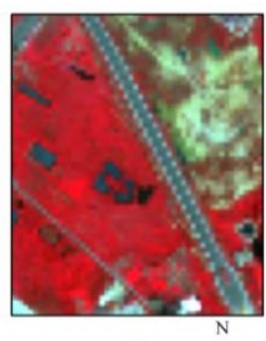

(5)

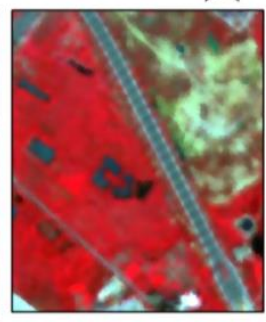

(10)

Fig. 8 (1) Original multispectral image as a reference with a spatial resolution of $2 \mathrm{~m}$. (2) Degraded multispectral image from (1) using nearest neighbor interpolation with a spatial resolution of $8 \mathrm{~m}$. (3, 4, and 5) Upgraded multispectral images from (2) using nearest neighbor, bilinear interpolation, and bicubic convolution, respectively. (6) The white rectangle is the position of the test data. (7) Degraded panchromatic image with a spatial resolution of $2 \mathrm{~m}$. (8, 9, and 10) Multispectral images reconstructed by proposed method from (7) and (3, 4, and 5) with a spatial resolution of $2 \mathrm{~m}$.
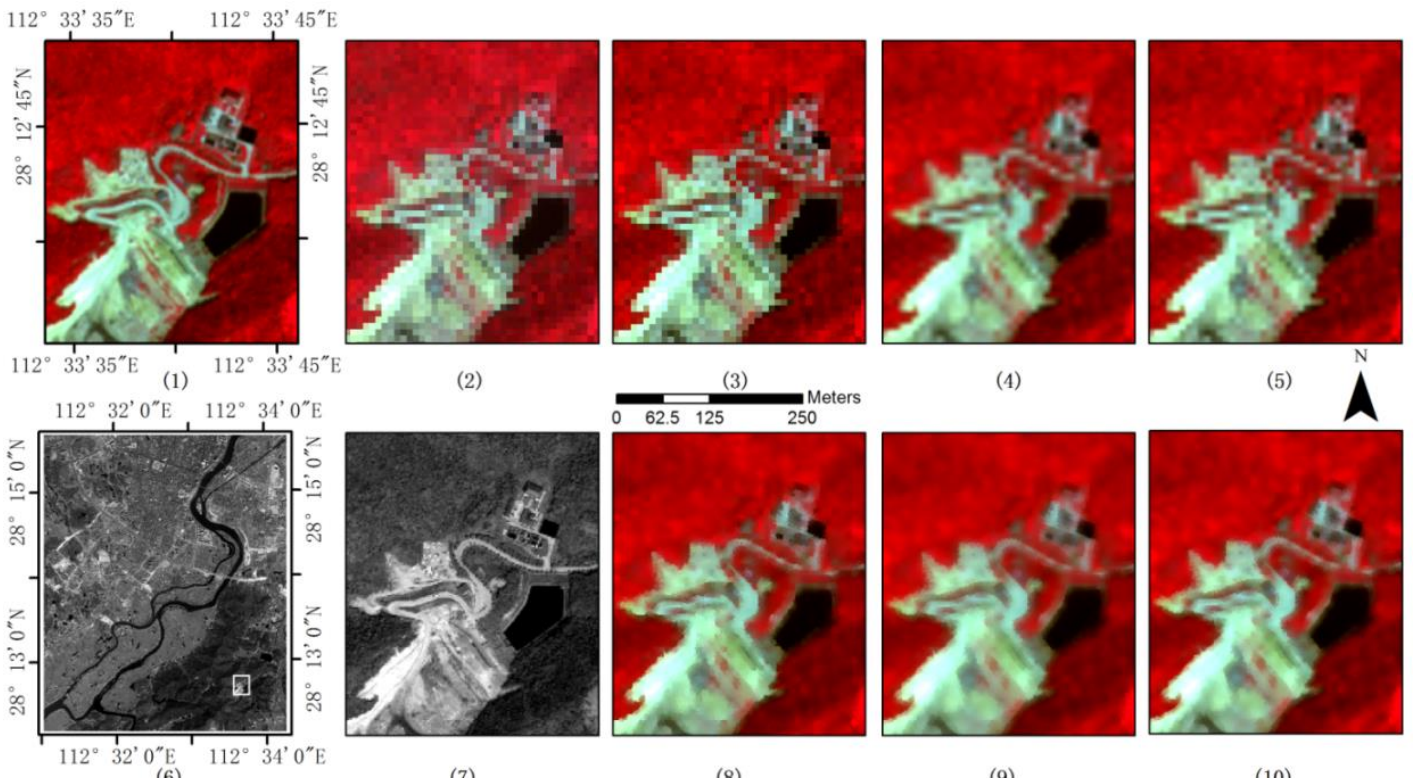

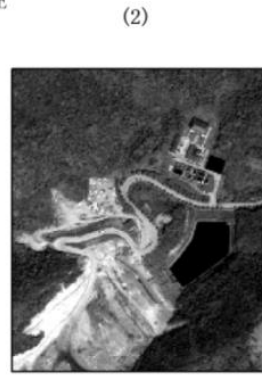

(7)

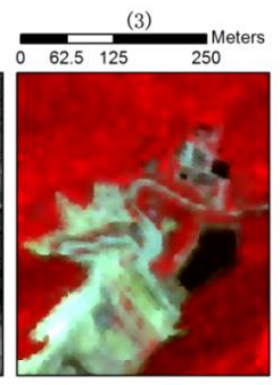

(8)

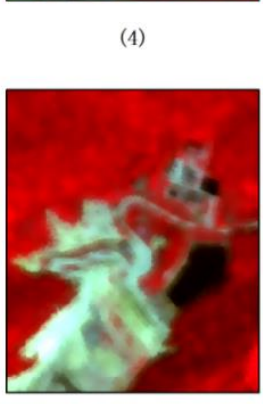

(9)

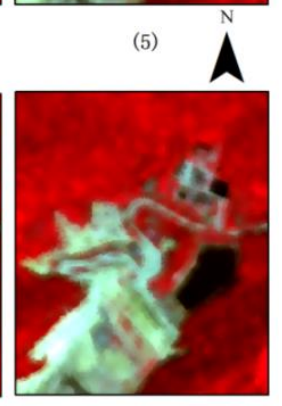

(10)

Fig. 9 (1) Original multispectral image as a reference with a spatial resolution of $2 \mathrm{~m}$. (2) Degraded multispectral image from (1) using nearest neighbor interpolation with a spatial resolution of $8 \mathrm{~m}$. (3, 4, and 5) Upgraded multispectral images from (2) using nearest neighbor, bilinear interpolation, and bicubic convolution, respectively. (6) The white rectangle is the position of the test data. (7) Degraded panchromatic image with a spatial resolution of $2 \mathrm{~m}$. (8, 9, and 10) Multispectral images reconstructed by proposed method from (7) and (3, 4, and 5) with a spatial resolution of $2 \mathrm{~m}$. 


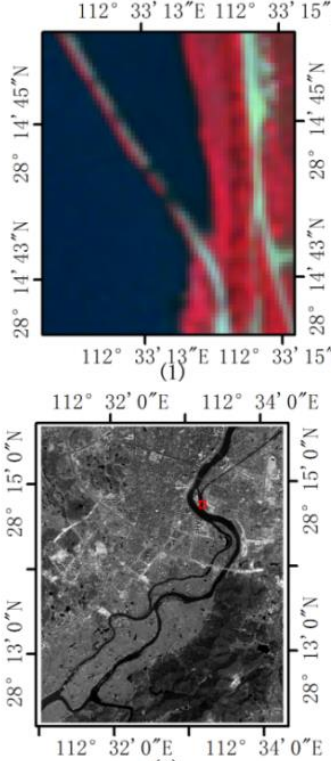

(6)

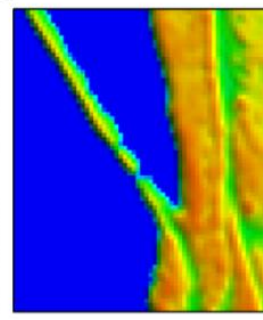

(2)

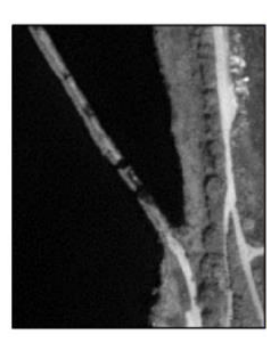

(7)
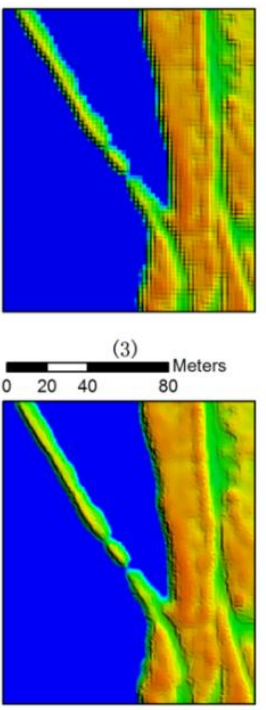

(8)

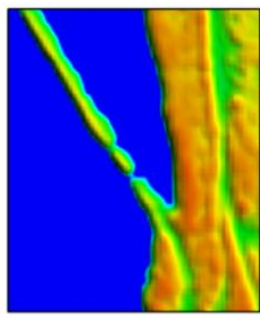

(4)

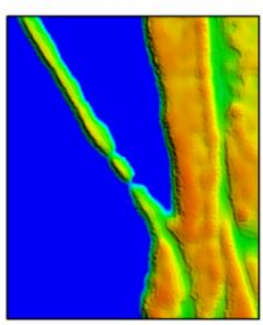

(9)

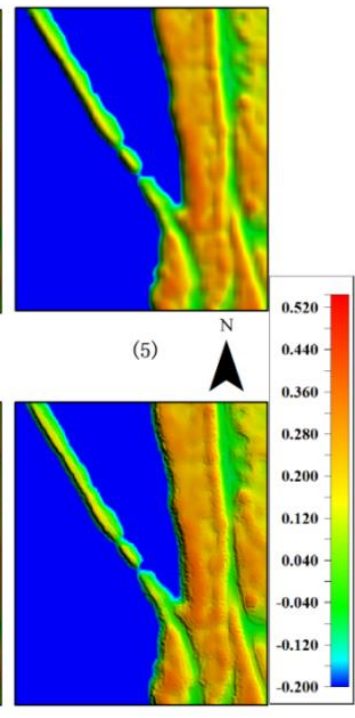

(10)

Fig. 10 (1) Original multispectral image with a spatial resolution of $2 \mathrm{~m}$. (2) NDVI image from (1). (3, 4, and 5) Upgraded NDVI images from (2) using nearest neighbor, bilinear interpolation, and bicubic convolution, respectively. (6) The red rectangle is the position of the test data. (7) Panchromatic image with a spatial resolution of $2 \mathrm{~m}$. (8, 9, and 10) NDVI images reconstructed by proposed method from (7) and (3, 4, and 5) with a spatial resolution of $2 \mathrm{~m}$. Note: For better visualization, NDVI images are simulated as terrain illumination (sun elevation $=$ $60^{\circ}$, and sun azimuth $=45^{\circ}$ ).

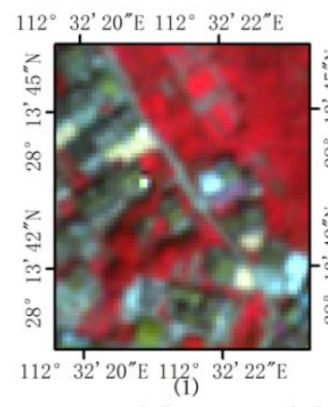

$112^{\circ} 32^{\prime} 0^{\prime \prime} \mathrm{E}, 112^{\circ} 34^{\prime} 0^{\prime \prime} \mathrm{E}$

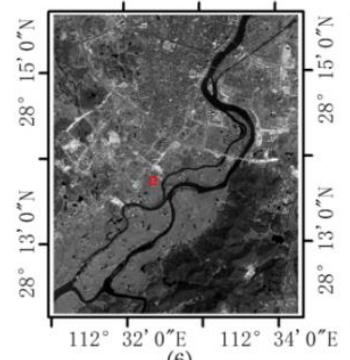

(6)

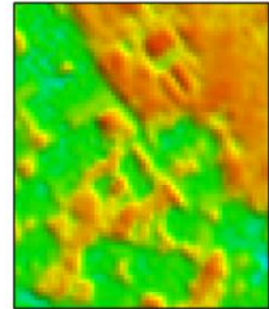

(2)

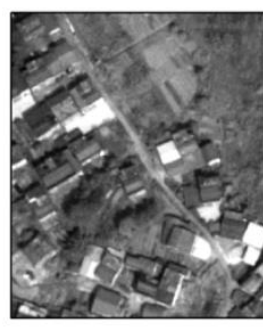

(7)
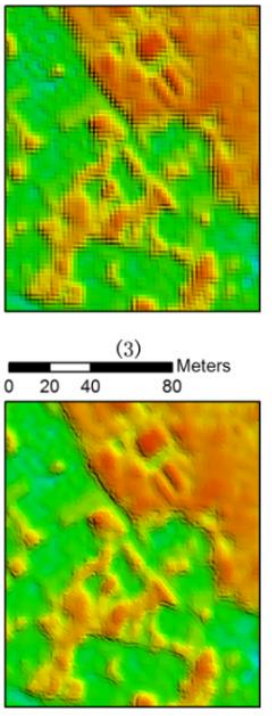

(8)

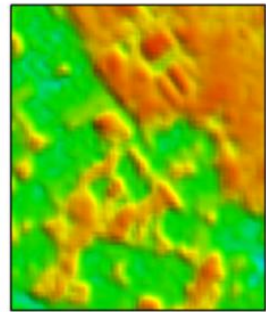

(4)

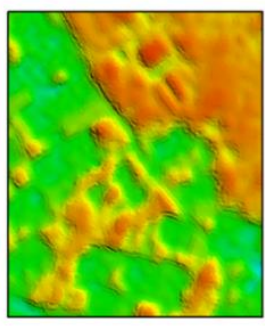

(9)

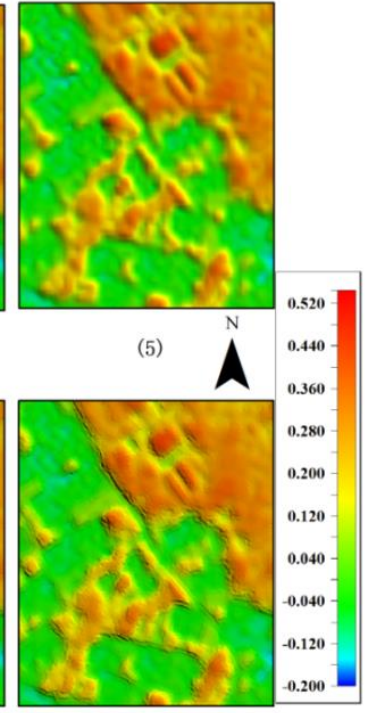

(10)

Fig. 11 (1) Original multispectral image with a spatial resolution of $2 \mathrm{~m}$. (2) NDVI image from (1). (3, 4, and 5) Upgraded NDVI images from (2) using nearest neighbor, bilinear interpolation, and bicubic convolution, respectively. (6) The red rectangle is the position of the test data. (7) Panchromatic image with a spatial resolution of $2 \mathrm{~m}$. (8, 9, and 10) NDVI images reconstructed by proposed method from (7) and (3, 4, and 5) with a spatial resolution of $2 \mathrm{~m}$. Note: For better visualization, NDVI images are simulated as terrain illumination (sun elevation = $60^{\circ}$, and sun azimuth $=45^{\circ}$ ). 

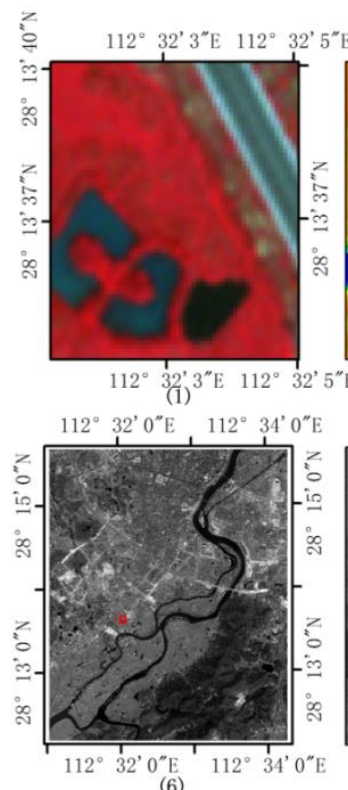

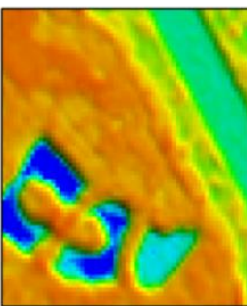

(2)

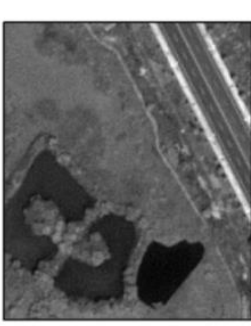

(7)

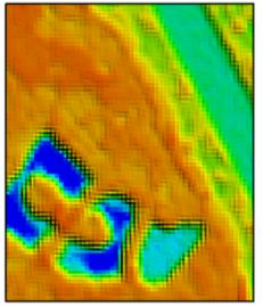

(3) Meters

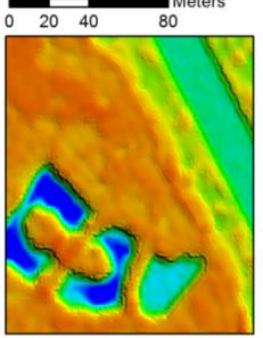

(8)

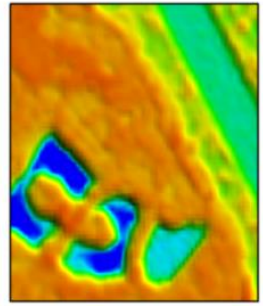

(4)

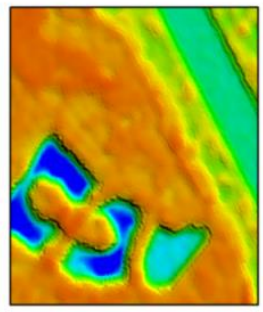

(9)

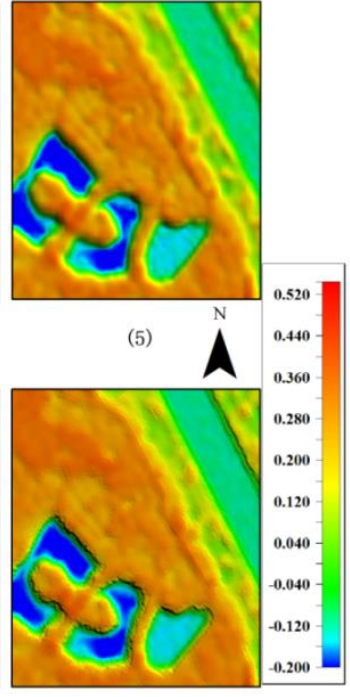

(10)

Fig. 12 (1) Original multispectral image with a spatial resolution of $2 \mathrm{~m}$. (2) NDVI image from (1). (3, 4, and 5) Upgraded NDVI images from (2) using nearest neighbor, bilinear interpolation, and bicubic convolution, respectively. (6) The red rectangle is the position of the test data. (7) Panchromatic image with a spatial resolution of $2 \mathrm{~m}$. (8, 9, and 10) NDVI images reconstructed by proposed method from (7) and (3, 4, and 5) with a spatial resolution of $2 \mathrm{~m}$. Note: For better visualization, NDVI images are simulated as terrain illumination (sun elevation $=$ $60^{\circ}$, and sun azimuth $=45^{\circ}$ ).
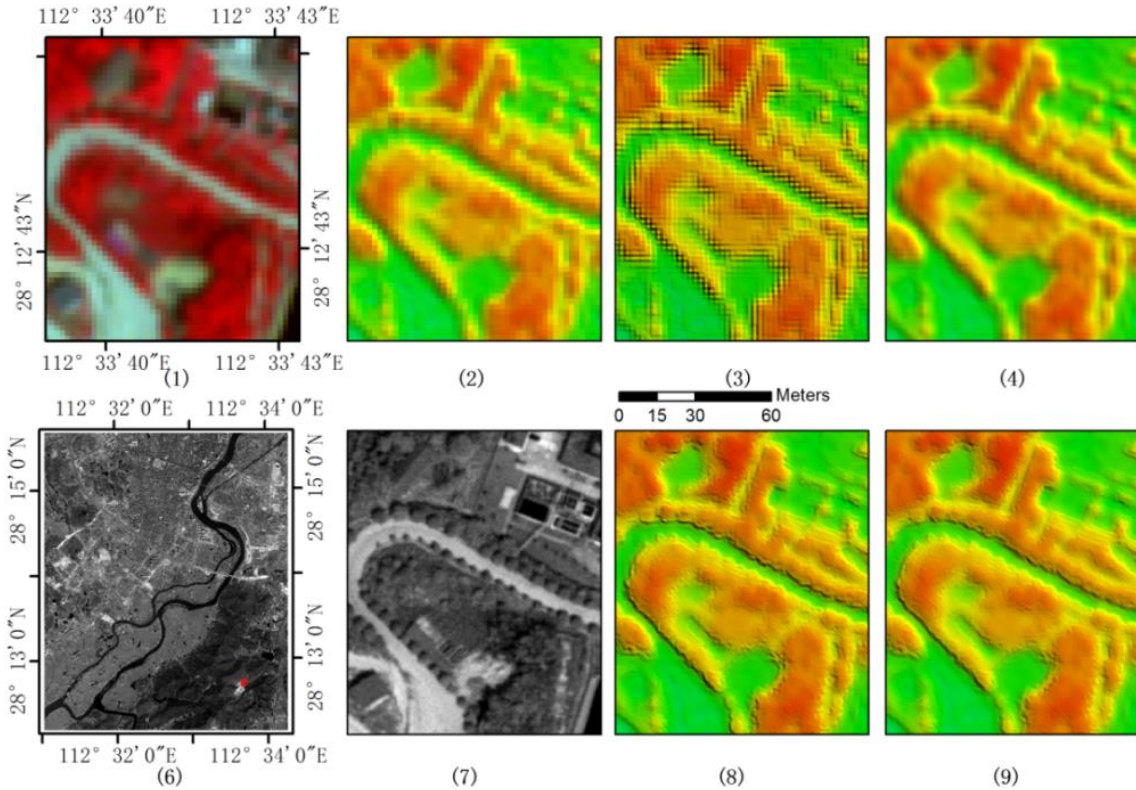

(7)

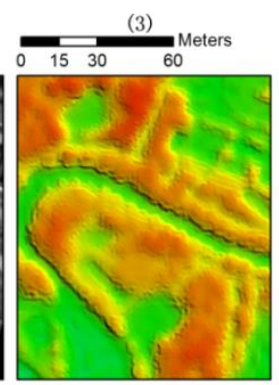

$(8)$
(4)

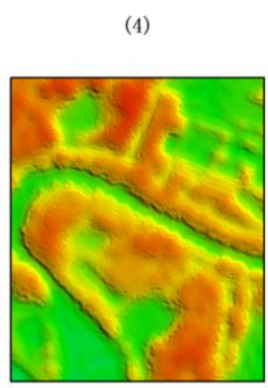

(9)

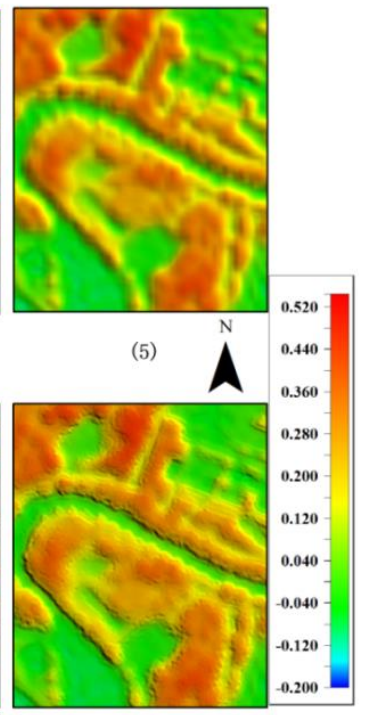

(10)

Fig. 13 (1) Original multispectral image with a spatial resolution of $2 \mathrm{~m}$. (2) NDVI image from (1). (3, 4, and 5) Upgraded NDVI images from (2) using nearest neighbor, bilinear interpolation, and bicubic convolution, respectively. (6) The red rectangle is the position of the test data. (7) Panchromatic image with a spatial resolution of $2 \mathrm{~m}$. (8, 9, and 10) NDVI images reconstructed by proposed method from (7) and (3, 4, and 5) with a spatial resolution of $2 \mathrm{~m}$. Note: For better visualization, NDVI images are simulated as terrain illumination (sun elevation $=$ $60^{\circ}$, and sun azimuth $=45^{\circ}$ ). 


\title{
Curriculum Vitae
}

\author{
Dengkui Mo \\ Forest Inventory and Remote Sensing Institute \\ Burckhardt Institute \\ Faculty of Forest Sciences and Forest Ecology \\ Georg-August-Universität Göttingen \\ Büsgenweg 5 \\ 37077 Göttingen, Germany \\ Phone: +49 [0]551 39-9831 \\ Email:dmo@gwdg.de; dengkuimo1980@gmail.com

\section{Personal Information} \\ Date \& Place of birth: November 23, 1980, Jingzhou, PR China \\ Nationality: Chinese
}

\section{Education}

Since 2013: PhD Program "Forest Science and Forest Ecology"

Georg-August-Universität Göttingen

2006-2012: Lecturer, teaching "Forest Resources Remote Sensing"

Central South University of Forestry and Technology

2003-2006: Master Program "Forest Inventory and Remote Sensing"

Central South University of Forestry and Technology

1999-2003: Bachelor Program "Forestry"

Central South Forestry University

\section{Main Research Interests}

1. Forest resources inventory, monitoring, and assessment

2. Intelligent image processing for remotely sensed images

3. GIS \& Spatial data analysis

4. Algorithm Design and Development

\section{Research Project Leader}

1. National Natural Science Foundation of China (Grant No. 31470643), 2011-2014

2. National Natural Science Foundation of China (Grant No. 31100412), 2015-2018

\section{Selected Publications}

[1] Dengkui Mo; Fuchs, H.; Fehrmann, L.; Haijun Yang; Yuanchang Lu; Kleinn, C., "Local Parameter Estimation of Topographic Normalization for Forest Type Classification," in Geoscience and Remote Sensing Letters, IEEE , vol.12, no.9, pp.1998-2002, Sept. 2015

[2] Dengkui Mo; Fuchs, H.; Fehrmann, L.; Haijun Yang; Kleinn, C.; Yuanchang Lu, "Moving window-based topographic normalization of optical satellite imagery for forest mapping in mountainous terrain," Earth Observation and Remote Sensing Applications (EORSA), 2014 3rd 
International Workshop on, vol., no., pp.452,456, 11-14 June 2014

[3] Dengkui Mo; Enping Yan; Hui Lin; Jiping Li; Guozhen Zhang, "A study on inland water quality parameters estimation and mapping using Hyperion imagery," Measurement, Information and Control (MIC), 2012 International Conference on, vol.1, no., pp.21,25, 18-20 May 2012

[4] Dengkui Mo; Enping Yan; Hui Lin; Hua Sun; Jiping Li; Guozhen Zhang, "Development and validation of 2-band EVI with MODIS data in Southeast China," Measurement, Information and Control (MIC), 2012 International Conference on , vol.1, no., pp.88,91, 18-20 May 2012

[5] Xiong, Y. J.,Qiu, G. Y., Mo, D. K., etc. Rocky desertification and its causes in karst areas: a case study in Yongshun County, Hunan Province, China[J]. Environmental Geology, 2009, 57 (7), pp 1481-1488.

[6] Mo, D. K.,Lin, H.,Sun, H., etc. A novel remotely sensed image interpretation method - MSSVMS[A], In Tianjin, China, 2009; pp 517-521.

[7] Mo, D. K. , Lin, H.,Li, J., etc. A SVM-based change detection method from bi-temporal remote sensing images in forest area[A], In Piscataway, NJ 08855-1331, United States, 2008; pp 209-212.

[8] Mo, D. K., Lin, H.,Li, J. P., etc. Remotely sensed imagery intelligent interpretation based on image segmentation and support vector machines - art. no. 67520N. Geoinformatics 2007: Remotely Sensed Data and Information, Pts 1 and 2, 2007, 6752 pp N7520-N7520.

[9] Mo, D. K.,Lin, H.. Study on the dynamic changes in rocky desertification in Yunshun County based on RS in northwestern Hunan Province. Principles and Practices of Desertification Control, Vol I, 2007, pp 395-403.

[10] Mo, D. K.,Lin, H.,Lv, Y., etc. Novel land cover classification based on mean shift segmentation for high resolution remote sensing. Proceedings of 2006 International Conference on Artificial Intelligence, 2006, pp 716-719.

[11] Mo, D. K. ,Lin, H.,Sun, H. , etc. Object oriented information extraction of forest resources from high resolution remote sensing - art. no. 641916. Geoinformatics 2006: Remotely Sensed Data and Information, 2006, 6419 pp 41916-41916.

[12] Mo, D. K.,Lin, H.,Li, J., etc. Design and implementation of a high spatial resolution remote sensing image intelligent interpretation system[J]. Data Science Journal, 2007, 6 (SUPPL),pp 445-452.

[13] Mo Dengkui, Lin Hui, Li Jiping. VHR Imagery Multi-Resolution Segmentation Based on Mean Shift (English)[J]. Journal of Guangxi Normal University (Natural Science Edition), 2006,(04),pp 247-250. 